\title{
Supersymmetry Flows, Semi-Symmetric Space Sine-Gordon Models And The Pohlmeyer Reduction
}

\author{
David M. Schmidtt \\ Instituto de Física Teórica - IFT/UNESP \\ Rua Dr. Bento Teobaldo Ferraz, 271, Bloco II \\ CEP 01140-070, São Paulo, SP, Brasil.
}

May 6, 2018

\begin{abstract}
We study the extended supersymmetric integrable hierarchy underlying the Pohlmeyer reduction of superstring sigma models on semi-symmetric superspaces $F / G$. This integrable hierarchy is constructed by coupling two copies of the homogeneous integrable hierarchy associated to the loop Lie superalgebra extension $\widehat{\mathfrak{f}}$ of the Lie superalgebra $\mathfrak{f}$ of $F$ and this is done by means of the algebraic dressing technique and a Riemann-Hilbert factorization problem. By using the Drinfeld-Sokolov procedure we construct explicitly, a set of $2 \mathrm{D}$ spin $\pm 1 / 2$ conserved supercharges generating supersymmetry flows in the phase space of the reduced model. We introduce the bi-Hamiltonian structure of the extended homogeneous hierarchy and show that the two brackets are of the Kostant-Kirillov type on the co-adjoint orbits defined by the light-cone Lax operators $L_{ \pm}$. By using the second symplectic structure, we show that these supersymmetries are Hamiltonian flows, we compute part of the supercharge algebra and find the supersymmetric field variations they induce. We also show that this second Poisson structure coincides with the canonical Lorentzinvariant symplectic structure of the WZNW model involved in the Lagrangian formulation of the extended integrable hierarchy, namely, the semi-symmetric space sine-Gordon model (SSSSG), which is the Pohlmeyer reduced action functional for the transverse degrees of freedom of superstring sigma models on the cosets $F / G$. We work out in some detail the Pohlmeyer reduction of the $A d S_{2} \times S^{2}$ and the $A d S_{3} \times S^{3}$ superstrings and show that the new conserved supercharges can be related to the supercharges extracted from $2 \mathrm{D}$ superspace. In particular, for the $A d S_{2} \times S^{2}$ example, they are formally the same.
\end{abstract}

\section{Contents}

1 Introduction.

2 General analysis.

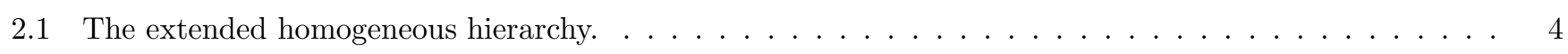

2.2 Relativistic sector of the extended homogeneous hierarchy. . . . . . . . . . . . . 8

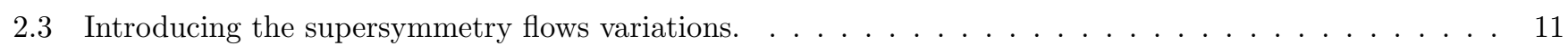


2.4 Lagrangian formulation of the semi-symmetric space sine-Gordon models. . . . . . . . . . . . . . 13

2.5 The Drinfeld-Sokolov procedure: the $\operatorname{dim} \mathcal{K}_{F}^{( \pm 1 / 2)} 2 \mathrm{D}$ spin $\pm 1 / 2$ supercharges. . . . . . . . . . 16

2.6 Bi-Hamiltonian structure of the extended homogeneous hierarchy. . . . . . . . . . . . . . 21

2.7 The second Hamiltonian structure: the supercharges algebra and the field variations. . . . . . . . . . 25

3 Pohlmeyer reduction of GS superstring sigma models.

4 Examples: supercharges from superspace and from symmetry flows. 34

4.1 Supercharges of the Landau-Ginzburg models. . . . . . . . . . . . . . . . . . . . . 34

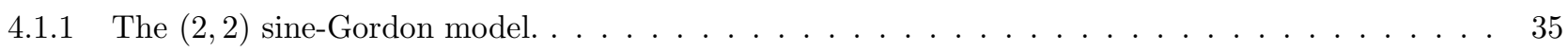

4.1.2 The $(2,2)$ complex sine-Gordon and its hyperbolic counterpart. . . . . . . . . . . . 35

4.2 Supercharges of the Pohlmeyer reduced models. . . . . . . . . . . . . . . . . . 37

4.2.1 Reduction of the $A d S_{2} \times S^{2}$ superstring and $(2,2) 2$ D SUSY . . . . . . . . . . 37

4.2.2 Reduction of the $A d S_{3} \times S^{3}$ superstring and possible $(4,4) 2$ D SUSY . . . . . . . . . . . 38

5 Concluding remarks.

6 Appendix A: The superalgebra $p s u(1,1 \mid 2) \quad 43$

$6.1 \mathbb{Z}_{4}$ grading and $\mathfrak{f}^{\perp}, \mathfrak{f}^{\|}$decomposition of $p s u(1,1 \mid 2) \ldots \ldots \ldots \ldots \ldots \ldots$

$6.2 \mathbb{Z}_{4}$ grading and $\mathfrak{f}^{\perp}, \mathfrak{f}^{\|}$decomposition of $p s u(1,1 \mid 2)^{\times 2} \ldots \ldots \ldots \ldots \ldots \ldots$

7 Appendix B: Relevant quantities used in the computations 47 


\section{Introduction.}

Recently [1], Grigoriev and Tseytlin motivated by a desired to find a useful 2D Lorentz-invariant reformulation of the classical-integrable $A d S_{5} \times S^{5}$ Green-Schwarz (GS) superstring world-sheet theory in terms of physical/transverse degrees of freedom only, constructed the Pohlmeyer reduced version of the $A d S_{5} \times S^{5}$ coset sigma model action. The corresponding reduced Lagrangian is of a non-Abelian Toda type: a gauged WZNW model with an integrable potential coupled to a set of 2D fermionic fields. Some of the main features of the reduced action is that it is Lorentz-invariant, the small-fluctuation spectrum near the trivial vacuum has the same number of bosonic and fermionic degrees of freedom and its integrable structure is equivalent to that of the initial sigma model. The structure of the reduced action suggest the presence of 2D supersymmetry, a fact that was confirmed explicitly for the simplest case of the sigma model on $A d S_{2} \times S^{2}$, which turned out to be equivalent to the $(2,2)$ supersymmetric extension of the sine-Gordon model [1].

In 2] were discussed the possible existence of hidden 2D supersymmetry in the first non-trivial reduced model which corresponds to the GS sigma model in the $A d S_{3} \times S^{3}$ background. The reduced action seems to be a $(2,2)$ supersymmetric extension of the complex sine-Gordon coupled in a non-trivial way with its hyperbolic counterpart, i.e the $(2,2)$ complex sinh-Gordon model, but its explicit superspace structure could not been identified as was done in the $A d S_{2} \times S^{2}$ case.

The Lie algebraic structure behind the Pohlmeyer reduction goes beyond the $A d S_{5} \times S^{5}$ case and is common to other sigma models and such a reduction can be performed, in principle and without major complications, on any GS superstring sigma model on a semi-symmetric superspace $F / G$, in which the Lie algebra $\mathfrak{g}$ of $G$ is the zero locus of a $\mathbb{Z}_{4}$ automorphism of the Lie superalgebra $\mathfrak{f}$ of $F$. However, despite of the simplicity for constructing the reduced models, there are still a number of open problems yet to be solved at the classical level, see for instance [1, [2]. Among them and the one we are most interested here is related to the conjectured existence of world-sheet supersymmetry in the reduced models [1], which have resisted to go beyond the simplest case $A d S_{2} \times S^{2}$ and remains as a non-trivial open question. It would be surprising to find it because of the initial sigma model is of GS type anyway.

In the present contribution, we start to study this question from the point of view of integrable systems, providing some evidence supporting such a conjecture. The strategy will be to identify the integrable structure behind the Pohlmeyer reduction process and use it to identify the would-be 2D world-sheet supersymmetry with the fermionic symmetry flows already present in the underlying integrable hierarchy. The outcome is that the $2 \mathrm{D}$ supersymmetry is associated to a special loop superalgebra $\widehat{\mathfrak{f}}^{\perp} \subset \widehat{\mathfrak{f}}$ constructed out of a subalgebra $\mathfrak{f}^{\perp} \subset \mathfrak{f}$ by means of the dressing flow transformations.

The outline of the paper is as follows. The chapter 2 is the main one and includes the general results. In section 2.1, we introduce the extended homogeneous hierarchy by using the dressing group and a Riemann-Hilbert factorization problem. The hierarchy is defined in terms of three gradations: the homogeneous gradation, associated to the loop

extension $\widehat{\mathfrak{f}}$ of the superalgebra $\mathfrak{f}$, the natural $\mathbb{Z}_{4}$ gradation of $\mathfrak{f}$, responsible for the matching of the physical degrees of freedom in the reduced model and a $\mathbb{Z}_{2}$ gradation, responsible for the consistency of the symmetry flows induced by a special sub-superalgebra $\mathfrak{f}^{\perp}$ of $\mathfrak{f}$. It is also shown how the usual gauge transformations can be interpreted as the lowest symmetry flows of the extended integrable hierarchy. In section 2.2, we define the relativistic sector of the hierarchy, which provides the Lax operators governing the Pohlmeyer reduced models. In section 2.3, we make a first tentative to introduce the $2 \mathrm{D}$ supersymmetry flows of the hierarchy, where some obstructions related to the locality of the gauge group are mentioned. In section 2.4, we make a review of the Lagrangian formulation of the relativistic sector of the hierarchy, i.e we introduce the semi-symmetric space sine-Gordon model (SSSSG), which is the action functional for the physical degrees of freedom in the reduction of GS sigma models. In section 2.5, we use the Drinfeld-Sokolov procedure to construct explicitly a set of $\operatorname{dim} \mathfrak{f}_{1,3}^{\perp} 2 \mathrm{D}$ spin $\pm 1 / 2$ conserved supercharges associated to the fermionic symmetry 
flows generated by the odd elements of $\mathfrak{f}_{1,3}^{\perp}$ of $\mathfrak{f}^{\perp}$. In section 2.6 , we show that the extended homogeneous hierarchy is bi-Hamiltonian in which the two symplectic structures take the form of Kostant-Kirillov brackets on the co-adjoint orbits defined by the Lax operators $L_{ \pm}$introduced in section 2.2. In section 2.7 , by using the second bracket, we compute part of the supercharge algebra, deduce the poisson form of the supersymmetry flow variations for the fields showing that they are hamiltonian flows in the Pohlmeyer reduced phase space and also mention on a subtlety related to the presence of the gauge group in the supercharge algebra. It is also shown that the second symplectic structure is equivalent to the canonical symplectic structure of the WZNW model involved in the construction of the SSSSG models. In chapter 3, we make a fast review of the Pohlmeyer reduction process in order to show how everything fits in the construction presented in chapter 2. In chapter 4 we work out explicit examples with the aim of exploring, in a first approximation, the relation between some well-known superspace results with the supersymmetry flow approach we have adopted. Finally, we make the concluding remarks and pose what will be done in the near future. There are two appendices including some technical details used in the computations. We have included some previous known results in parts of the body of the paper with the aim of render it as self-contained as possible.

\section{General analysis.}

This is the main chapter and includes all the results of the paper. The idea is to introduce and study the integrable supersymmetric hierarchy underlying the Pohlmeyer reduction of superstring sigma models. The most important result is the explicit construction of the supercharges generating 2D Hamiltonian fermionic symmetry flows on the phase space of the reduced models, see (68) below.

\subsection{The extended homogeneous hierarchy.}

Here we show how to locate gauge symmetries in the context of the algebraic dressing technique. We refine the results of [6] in order address later the situation we are most interested, namely the Pohlmeyer reduction of superstring sigma models.

Start by considering a finite dimensional Lie superalgebra $\mathfrak{f}$ endowed with an order four linear automorphism $\Omega$, $\Omega: \mathfrak{f} \rightarrow \mathfrak{f}, \Omega([X, Y])=[\Omega(X), \Omega(Y)], \Omega^{4}=I$. The superalgebra $\mathfrak{f}$ then admits a $\mathbb{Z}_{4}$ grade space decomposition

$$
\mathfrak{f}=\mathfrak{f}_{0} \oplus \mathfrak{f}_{1} \oplus \mathfrak{f}_{2} \oplus \mathfrak{f}_{3}
$$

which is consistent with the (anti)-commutation relations $\left[\mathfrak{f}_{i}, \mathfrak{f}_{j}\right] \subset \mathfrak{f}_{(i+j) \bmod 4}$. The subspace $\mathfrak{f}_{j}$ is formed by the elements of $\mathfrak{f}$ with $\mathbb{Z}_{4}$ grading $j, \Omega\left(\mathfrak{f}_{j}\right)=(i)^{j} \mathfrak{f}_{j}$. The even (or bosonic) subalgebra is $\mathfrak{f}_{B}=\mathfrak{f}_{0} \oplus \mathfrak{f}_{2}$ while the odd (or fermionic) part of $\mathfrak{f}$ is formed by $\mathfrak{f}_{F}=\mathfrak{f}_{1} \oplus \mathfrak{f}_{3}$.

We need to introduce a semisimple element $\Lambda \in \mathfrak{f}_{2}$ which induces the following superalgebra spliting

$$
\mathfrak{f}=\mathfrak{f}^{\perp} \oplus \mathfrak{f}^{\|}, \quad \mathfrak{f}^{\perp} \cap \mathfrak{f}^{\|}=\oslash,
$$

where $\mathfrak{f}^{\perp} \equiv \operatorname{ker}(\operatorname{ad}(\Lambda))$ and $\mathfrak{f}^{\|} \equiv \operatorname{Im}(\operatorname{ad}(\Lambda))$.

We restric ourselves to the situation in which $\mathfrak{f}$ admits an extra $\mathbb{Z}_{2}$ gradation $\sigma: \mathfrak{f} \rightarrow \mathfrak{f}, \sigma([X, Y])=[\sigma(X), \sigma(Y)]$, $\sigma^{2}=I$ with $\sigma\left(\mathfrak{f}^{\perp}\right)=\mathfrak{f}^{\perp}$ and $\sigma\left(\mathfrak{f}^{\|}\right)=-\mathfrak{f}^{\|}$, implying that $\mathfrak{f}$ is also a symmetric space 1

$$
\left[\mathfrak{f}^{\perp}, \mathfrak{f}^{\perp}\right] \subset \mathfrak{f}^{\perp}, \quad\left[\mathfrak{f}^{\perp}, \mathfrak{f}^{\|}\right] \subset \mathfrak{f}^{\|}, \quad\left[\mathfrak{f}^{\|}, \mathfrak{f}^{\|}\right] \subset \mathfrak{f}^{\perp} .
$$

\footnotetext{
${ }^{1}$ In particular, this is satisfied by the superalgebras entering the Pohlmeyer reduction of $A d S_{n} \times S^{n}, n=2,3,5$ and $A d S_{4} \times \mathbb{C} P^{3}$ superstring sigma models. The only exception is $n=2$, which has $\mathfrak{f}_{0}^{\perp}=\varnothing$.
} 
The algebraic structure underlying the integrable hierarchy we are interested in, is defined by the following graded loop Lie superalgebra

$$
\widehat{\mathfrak{f}}=\bigoplus_{n \in \mathbb{Z}=-\infty}^{+\infty}\left(\bigoplus_{j \in \mathbb{Z}=0}^{3} z^{2 n+\frac{j}{2}} \otimes \mathfrak{f}_{j}\right),
$$

which can be rewriten as a half-integer decomposition

$$
\widehat{\mathfrak{f}}=\bigoplus_{r \in \mathbb{Z} / 2=-\infty}^{+\infty} \widehat{\mathfrak{f}}_{r}, \quad\left[Q, \widehat{\mathfrak{f}}_{r}\right]=r \widehat{\mathfrak{f}}_{r}
$$

in terms of the homogeneous gradation $Q=z \frac{d}{d z}$. The complex variable $z$ will enter later in the Lax operators as the spectral parameter and it is worth to note that under $Q$, the integer and half-integer elements of $\widehat{\mathfrak{f}}$ are, respectively, bosonic and fermionic in character.

The splitting (2) is now lifted to the affine algebra $\widehat{\mathfrak{f}}$ which we write in the form

$$
\widehat{\mathfrak{f}}=\mathcal{K} \oplus \mathcal{M}
$$

where $\mathcal{K}=\mathcal{K}_{B} \oplus \mathcal{K}_{F}=\operatorname{ker}\left(a d\left(\Lambda^{( \pm 1)}\right)\right), \mathcal{M}=\mathcal{M}_{B} \oplus \mathcal{M}_{F}=\operatorname{Im}\left(\operatorname{ad}\left(\Lambda^{( \pm 1)}\right)\right)$ and $\left[Q, \Lambda^{( \pm 1)}\right]= \pm \Lambda^{( \pm 1)}$. In what follows the superscript $r$ of an element $X^{(r)} \in \widehat{\mathfrak{f}}_{r}$ stands for the homogeneous grading $\left[Q, X^{(r)}\right]=r X^{(r)}, r \in \mathbb{Z} / 2$ and projections along $\mathcal{K}$ and $\mathcal{M}$ will be denoted by $(*)^{\perp}$ and $(*)^{\|}$, respectively. As above, we have

$$
[\mathcal{K}, \mathcal{K}] \subset \mathcal{K}, \quad[\mathcal{K}, \mathcal{M}] \subset \mathcal{M}, \quad[\mathcal{M}, \mathcal{M}] \subset \mathcal{K} .
$$

This homogeneous half-integer gradation is enough for all our purpose 2 , namely, to introduce the symmetry flows, to deduce the Lax operators and to extract the conserved charges. The first and second relations in (5) are at the heart of the implementation of symmetry flows by means of the algebraic dressing technique in which the symmetries are associated to the subalgebra $\mathcal{K}$ while the dynamical physical fields are associated to $\mathcal{M}$ inducing a mapping $\delta_{\mathcal{K}}: \mathcal{M} \rightarrow \mathcal{M}$ from physical fields to physical fields. This is also the algebraic setting behind the Drinfeld-Sokolov procedure we shall use later.

We now proceed to make this construction more precise. Decompose (4) as $\widehat{\mathfrak{f}}=\widehat{\mathfrak{f}}_{-}+\widehat{\mathfrak{f}}_{+}$, where $\widehat{\mathfrak{f}}_{ \pm}$are the positive and negative subalgebras induced by the gradation $Q$ and introduce the following loop supergroup matrices: the so-called dressing matrices

$$
\begin{aligned}
& \Theta=\exp \left(\chi^{(-1 / 2)}+\chi^{(-1)}+\chi^{(-3 / 2)}+\ldots\right), \quad \Theta^{\prime}=B^{-1} \Theta, \\
& \Pi=B \Pi^{\prime}, \quad \Pi^{\prime}=\exp -\left(\chi^{(+1 / 2)}+\chi^{(+1)}+\chi^{(+3 / 2)}+\ldots\right),
\end{aligned}
$$

where $B \in G=\exp \widehat{\mathfrak{f}}_{0}$ is the Toda field, $\chi^{(r)}=\psi^{(r)}+\theta^{(r)} \in \widehat{\mathfrak{f}}_{r}, \theta^{(r)} \in \widehat{\mathfrak{f}}_{r}^{\perp}$ and $\psi^{(r)} \in \widehat{\mathfrak{f}}_{r}^{\|}$are related to the matter fields. Note that $B$ appears in two different positions and this will be very useful later.

Recall [3] that the dressing transformation of $x \in \widehat{F}$ by $g \in \widehat{F}$ is defined by ${ }^{g} x=\left(x g x^{-1}\right)_{ \pm} x g_{ \pm}^{-1}$, where $g=g_{-}^{-1} g_{+}$. For an element $g=\exp A$ with $A=A_{+}+A_{-}$and $A_{ \pm} \in \widehat{\mathfrak{f}}_{ \pm}$, the infinitesimal dressing transformation is

$$
\delta_{A} x={ }^{g} x-x= \pm\left(x A x^{-1}\right)_{ \pm} x \mp x A_{ \pm} .
$$

We are interested in using the kernel subalgebra $\mathcal{K}$ to generate actions on the dressing matrices which are carrying the dynamical degrees of freedom. From (7) and the decomposition $\mathcal{K}=\mathcal{K}_{-}+\mathcal{K}_{+}$, we find the infinitesimal actions of $A=A_{+} \in \mathcal{K}_{+}$and $A=A_{-} \in \mathcal{K}_{-}$on $x=\Theta$ and $x=\Pi$, respectively

$$
\delta_{A_{+}} \Theta=-\left(\Theta A_{+} \Theta^{-1}\right)_{-} \Theta, \quad \delta_{A_{-}} \Pi=+\left(\Pi A_{-} \Pi^{-1}\right)_{+} \Pi,
$$

\footnotetext{
${ }^{2}$ This is why we have chosen the name homogeneous integrable hierarchy in this paper.
} 
where $A_{ \pm}$are linear combinations of elements of $\mathcal{K}_{ \pm}$and $(*)_{ \pm}$stands for projections $\mathcal{P}_{ \pm}$along $\widehat{\mathfrak{f}}_{ \pm}$. It is not difficult to show that for $A=A_{-}$and $x=\Theta$ and for $A=A_{+}$and $x=\Pi$ the variations vanish, $\delta_{A_{-}} \Theta=0$ and $\delta_{A_{+}} \Pi=0$. Hence, in the present form, the dressing matrices (6) only evolve under half of the kernel algebra $\mathcal{K}$ and we have two sets of decoupled evolution equations.

Taking $A_{ \pm}=t_{ \pm n} \Lambda^{( \pm n)}, n \in \mathbb{Z}^{+}$, where $\Lambda^{( \pm n)} \in \operatorname{Cent}(\mathcal{K})$ belong to the center of $\mathcal{K}$ and taking the limit $t_{ \pm n} \rightarrow 0$ we obtain 3 the isospectral evolutions of $\Theta$ and $\Pi$

$$
\partial_{+n} \Theta=-\left(\Theta \Lambda^{(+n)} \Theta^{-1}\right)_{-} \Theta, \quad \partial_{-n} \Pi=+\left(\Pi \Lambda^{(-n)} \Pi^{-1}\right)_{+} \Pi .
$$

From equations (9) we obtain the Lax connections

$$
\Lambda_{\Theta}^{(+n)}=\left(\Theta \Lambda^{(+n)} \Theta^{-1}\right)_{+}, \quad \Lambda_{\Pi}^{(-n)}=\left(\Pi \Lambda^{(-n)} \Pi^{-1}\right)_{-}
$$

and the Lax operators $L_{+n}=\partial_{+n}-\Lambda_{\Theta}^{(+n)}, L_{-n}=\partial_{-n}+\Lambda_{\Pi}^{(-n)}$ from the dressing relations

$$
L_{-n}=\Pi L_{-n}^{V} \Pi^{-1}, \quad L_{+n}=\Theta L_{+n}^{V} \Theta^{-1}
$$

where $L_{ \pm n}^{V}=\partial_{ \pm n} \mp \Lambda^{( \pm n)}$ are the vaccum Lax operators. The Baker-Akhiezer wave functions $\Psi_{ \pm}$are defined by $L_{ \pm n} \Psi_{\mp}=0$ and are given by

$$
\Psi_{-}=\Theta \exp \left(+\sum_{n \in \mathbb{Z}^{+}} t_{+n} \Lambda^{(+n)}\right), \quad \Psi_{+}=\Pi \exp \left(-\sum_{n \in \mathbb{Z}^{+}} t_{-n} \Lambda^{(-n)}\right) .
$$

The equations (9) describe two identical but decoupled sets of evolution equations as we mentioned above, the coupling of the two sectors (of positive and negative times) is achieved by imposing the relation $g=\Psi_{-}^{-1} \Psi_{+}$with $g=g_{-}^{-1} g_{+} \in \widehat{F}$ a constant loop group element. Alternatively, we have (see also [4])

$$
\exp \left(+\sum_{n \in \mathbb{Z}^{+}} t_{+n} \Lambda^{(+n)}\right) g \exp \left(+\sum_{n \in \mathbb{Z}^{+}} t_{-n} \Lambda^{(-n)}\right)=\Theta^{-1}(t) \Pi(t) .
$$

This is the Riemann-Hilbert factorization problem we use in order to extend the associated integrable hierarchy described by (8) to flow now under the negative times. From (12) we recover (9) and two important extra equations describing the isospectral evolution of $\Theta$ and $\Pi$ with respect opposite flow parameters

$$
\partial_{+n} \Pi=+\left(\Theta \Lambda^{(+n)} \Theta^{-1}\right)_{+} \Pi, \quad \partial_{-n} \Theta=-\left(\Pi \Lambda^{(-n)} \Pi^{-1}\right)_{-} \Theta .
$$

These equations are extended to actions of $A_{+} \in \mathcal{K}_{+}$and $A_{-} \in \mathcal{K}_{-}$on $\Pi, \Theta$ and besides of (8) we also have now that

$$
\delta_{A_{+}} \Pi=+\left(\Theta A_{+} \Theta^{-1}\right)_{+} \Pi, \quad \delta_{A_{-}} \Theta=-\left(\Pi A_{-} \Pi^{-1}\right)_{-} \Theta .
$$

The equations (8), (9) and (13), (14) describe the isospectral evolution and non-Abelian variations of the dressing matrices $\Theta$ and $\Pi$. Note that the flows associated to the positive times are dual to the ones associated to the negative times, in the sense that $\mathcal{K}_{+}^{*} \simeq \mathcal{K}_{-}$under the (assumed to exists) non-degenerate inner product $\langle A, B\rangle$ on $\widehat{\mathfrak{f}}$ defined by

$$
\left\langle X^{(r)}, Y^{(s)}\right\rangle_{\widehat{f}}=\delta_{r+s, 0} \times \operatorname{Str}(X \cdot Y)_{\mathfrak{f}},
$$

where $X^{(r)}=z^{r} \otimes X_{r}, X_{r} \in \mathfrak{f}_{r}$ and $S t r$ is the supertrace in some supermatrix representation of $\mathfrak{f}$.

\footnotetext{
${ }^{3}$ Use $\delta_{A_{+}} \Theta / t_{+}=\left({ }^{A_{+} \Theta-\Theta}\right) / t_{+} \rightarrow \partial_{+n} \Theta$ and a similar expression for $\delta_{A_{-}} \Pi$.
} 
The decomposition in terms of grades is slightly ambiguous because we can take $\widehat{\mathfrak{f}}=\widehat{\mathfrak{f}}_{-}+\widehat{\mathfrak{f}}_{+}$with $\widehat{\mathfrak{f}}_{-}=\oplus_{r \leq-1 / 2}$ $\widehat{\mathfrak{f}}_{r}, \widehat{\mathfrak{f}}_{+}=\oplus_{r \geq 0} \widehat{\mathfrak{f}}_{r}$ or $\widehat{\mathfrak{f}}_{-}=\oplus_{r \leq 0} \widehat{\mathfrak{f}}_{r}, \widehat{\mathfrak{f}}_{+}=\oplus_{r \geq+1 / 2} \widehat{\mathfrak{f}}_{r}$ and this turn out to be related to gauge symmetries of the form $H_{L} \times H_{R}$, as we shall see below. For the moment let us take into account this difference in order to rewrite the symmetry flows more explicitly.

All the above evolution equations for $\Theta$ and $\Pi$ are summarized into the flow equations 4

$$
\begin{array}{ll}
\delta_{A_{+}} \Theta=-\left(\Theta A_{+} \Theta^{-1}\right)_{<0} \Theta, & \delta_{A_{-}} \Pi=+\left(\Pi A_{-} \Pi^{-1}\right)_{\geq 0} \Pi, \\
\delta_{A_{+}} \Pi=+\left(\Theta A_{+} \Theta^{-1}\right)_{\geq 0} \Pi, & \delta_{A_{-}} \Theta=-\left(\Pi A_{-} \Pi^{-1}\right)_{<0} \Theta,
\end{array}
$$

where we have chosen to put the grade zero part $\widehat{\mathfrak{f}}_{0}$ in $\widehat{\mathfrak{f}}_{+}$. Let us note that after the coupling the dynamical degrees of freedom are doubled by the extension because now we have two dressing matrices $\Theta, \Pi$ carrying different sets of fields.

The RHS of (12) can be written in an equivalent way because $\Theta^{-1} \Pi=\Theta^{\prime-1} \Pi^{\prime}$, cf (6). In these prime variables the equations (16) become

$$
\begin{array}{ll}
\delta_{A_{+}} \Theta^{\prime}=-\left(\Theta^{\prime} A_{+} \Theta^{\prime-1}\right)_{\leq 0} \Theta^{\prime}, & \delta_{A_{-}} \Pi^{\prime}=+\left(\Pi^{\prime} A_{-} \Pi^{\prime-1}\right)_{>0} \Pi^{\prime}, \\
\delta_{A_{+}} \Pi^{\prime}=+\left(\Theta^{\prime} A_{+} \Theta^{\prime-1}\right)_{>0} \Pi^{\prime}, & \delta_{A_{-}} \Theta^{\prime}=-\left(\Pi^{\prime} A_{-} \Pi^{\prime-1}\right)_{\leq 0} \Theta^{\prime},
\end{array}
$$

where we have chosen to put the grade zero part $\widehat{\mathfrak{f}}_{0}$ in $\widehat{\mathfrak{f}}_{-}$. From (17) we obtain the Lax connections

$$
\Lambda_{\Theta^{\prime}}^{(+n)}=\left(\Theta^{\prime} \Lambda^{(+n)} \Theta^{\prime-1}\right)_{>0}, \quad \Lambda_{\Pi^{\prime}}^{(-n)}=\left(\Pi^{\prime} \Lambda^{(-n)} \Pi^{\prime-1}\right)_{\leq 0}
$$

and the Lax operators $L_{+n}^{\prime}=\partial_{+n}-\Lambda_{\Theta^{\prime}}^{(+n)}, L_{-n}^{\prime}=\partial_{-n}+\Lambda_{\Pi^{\prime}}^{(-n)}$ from the dressing relations (11) with $\Theta$, $\Pi$ replaced by $\Theta^{\prime}, \Pi^{\prime}$. The prime and un-prime expressions make clear the projections to be used in computations. Of course, the two formulations are completely equivalent and it is not difficult to see that the Lax operators are related by a $B$-conjugation

$$
L_{ \pm n}^{\prime}=B^{-1} L_{ \pm n} B
$$

However, as we will see along the text this is not an ordinary gauge transformation because in the decomposition $\widehat{\mathfrak{f}}_{0}=\widehat{\mathfrak{f}}_{0}^{\perp}+\widehat{\mathfrak{f}}_{0}^{\|}$, the $\widehat{\mathfrak{f}}_{0}^{\perp}$ is the gauge algebra while in $B \in G=\exp \widehat{\mathfrak{f}}_{0}$ we have physical fields in $\widehat{\mathfrak{f}}_{0}^{\|}$.

In the mKdV hierarchy the grade zero part of the bosonic kernel is empty, i.e $\mathcal{K}_{B}^{(0)}=\widehat{\mathfrak{f}}_{0}^{\perp}=\oslash$ and this was the situation already considered in [6. Now we have that $\mathcal{K}_{B}^{(0)} \neq \varnothing$, which is more interesting. In this case we have symmetry flows associated to $\widehat{\mathfrak{f}}_{0}^{\perp}$. As mentioned before, this flows are nothing but usual gauge symmetries and now we proceed to identify them.

The $H_{L} \times H_{R}$ gauge transformations are generated by the elements $K_{L / R}^{(0)} \in \mathfrak{h}_{L} \times \mathfrak{h}_{R}=\widehat{\mathfrak{f}}_{0}^{\perp} \times \widehat{\mathfrak{f}}_{0}^{\perp}$ and their action are encoded in the following flow equations

$$
\begin{array}{rlrl}
\delta_{L} \Theta & =-\left(\Theta K_{L}^{(0)} \Theta^{-1}\right)_{<0} \Theta, & & \delta_{L} \Pi=+\left(\Theta K_{L}^{(0)} \Theta^{-1}\right)_{\geq 0} \Pi, \\
\delta_{R} \Theta^{\prime} & =-\left(\Pi^{\prime} K_{R}^{(0)} \Pi^{\prime-1}\right)_{\leq 0} \Theta^{\prime}, & \delta_{R} \Pi^{\prime}=+\left(\Pi^{\prime} K_{R}^{(0)} \Pi^{\prime-1}\right)_{>0} \Pi^{\prime} .
\end{array}
$$

Consider the first line of (20) and a constant element $K_{L}^{(0)} \in \mathfrak{h}_{L}$. These equations give rise to the dressing relations

$$
\Theta\left(\delta_{L}-K_{L}^{(0)}\right) \Theta^{-1}=\delta_{L}-K_{L}^{(0)}, \quad \Pi\left(\delta_{L}\right) \Pi^{-1}=\delta_{L}-K_{L}^{(0)} .
$$

\footnotetext{
${ }^{4}$ One of the most remarkable properties of the flow equations (16), (17) is that they associate a 2D symmetry flow to every Lie algebra generator in $\mathcal{K}$ through a Lax operator of the form $L_{\mathcal{K}}=\delta_{\mathcal{K}}+\mathcal{A}_{\mathcal{K}}$. The symmetry field variations are obtained by dressing the identities $\left[L_{\mathcal{K}}^{V}, L_{ \pm}^{V}\right]=0$, where $L_{\mathcal{K}}^{V}, L_{ \pm}^{V}$ are the vacuum Lax operators.
} 
The first equation is equivalent to the infinitesimal gauge transformations $\delta_{L} \Theta=\left[K_{L}^{(0)}, \Theta\right]$ and the second equation is equivalent to $\delta_{L} \Pi^{\prime} \Pi^{\prime-1}=0$ and $\delta_{L} B=K_{L}^{(0)} B$, where we have used $\Pi=B \Pi^{\prime}$. Setting $\Gamma_{L}=\exp K_{L}^{(0)} \in H_{L}=\exp \mathfrak{h}_{L}$, we get the finite gauge transformations

$$
\widetilde{\Theta}_{L}=\Gamma_{L} \Theta \Gamma_{L}^{-1}, \quad \widetilde{B}_{L}=\Gamma_{L} B, \quad{\widetilde{\Pi^{\prime}}}_{L}=\Pi^{\prime} .
$$

Under these left-gauge transformations the Lax connections $\Lambda_{\Theta}^{(+n)}, \Lambda_{\Pi}^{(-n)}$ transform as

$$
\Lambda_{\widetilde{\Theta}_{L}}^{(+n)}=\Gamma_{L} \Lambda_{\Theta}^{(+n)} \Gamma_{L}^{-1}, \quad \Lambda_{\widetilde{\Pi}_{L}}^{(-n)}=\Gamma_{L} \Lambda_{\Pi}^{(-n)} \Gamma_{L}^{-1}
$$

preserving the compatibility conditions because $\widetilde{F}_{m n}^{L}=\Gamma_{L} F_{m n} \Gamma_{L}^{-1}$. In an analogous way, considering the second line of (20) and an element $K_{R}^{(0)} \in \mathfrak{h}_{R}$ we have the dressing relations

$$
\Theta^{\prime}\left(\delta_{R}\right) \Theta^{\prime-1}=\delta_{R}+K_{R}^{(0)}, \quad \Pi^{\prime}\left(\delta_{R}+K_{R}^{(0)}\right) \Pi^{\prime-1}=\delta_{R}+K_{R}^{(0)},
$$

which are equivalent to the infinitesimal gauge transformations $\delta_{R} \Theta \Theta^{-1}=0, \delta_{R} B=B K_{R}^{(0)}$ and $\delta_{R} \Pi^{\prime}=-\left[K_{R}^{(0)}, \Pi^{\prime}\right]$. Setting $\Gamma_{R}=\exp K_{R}^{(0)} \in H_{R}=\exp \mathfrak{h}_{R}$, we get the finite gauge transformations

$$
\widetilde{\Theta}_{R}=\Theta, \quad \widetilde{B}_{R}=B \Gamma_{R}, \quad \widetilde{\Pi}_{R}^{\prime}=\Gamma_{R}^{-1} \Pi^{\prime} \Gamma_{R} .
$$

Under (22) the Lax connections $\Lambda_{\Theta}^{(+n)}, \Lambda_{\Pi}^{(-n)}$ are gauge invariant and we have $\widetilde{F}_{m n}^{R}=F_{m n}$. This is not an asymmetric behavior, if we perform the same analysis in the $B$-equivalent representation given by (17) with Lax connections $\Lambda_{\Theta^{\prime}}^{(+n)}$ , $\Lambda_{\Pi^{\prime}}^{(-n)}$, the situation is reversed, i.e $\widetilde{F}_{m n}^{\prime R}=\Gamma_{R}^{-1} F_{m n}^{\prime} \Gamma_{R}$ and $\widetilde{F}_{m n}^{\prime L}=F_{m n}^{\prime}$. This is a consequence of the position of the Toda field in (6).

Then, combining (21) and (22) we have the total finite action of the gauge group $H_{L} \times H_{R}$ on the dressing matrices

$$
\widetilde{\Theta}=\Gamma_{L} \Theta \Gamma_{L}^{-1}, \quad \widetilde{B}=\Gamma_{L} B \Gamma_{R}, \quad \widetilde{\Pi^{\prime}}=\Gamma_{R}^{-1} \Pi^{\prime} \Gamma_{R}
$$

and from (66) we get the action on each graded subspace $\psi^{( \pm r)} \in \widehat{\mathfrak{f}}_{ \pm r}$. In particular and for future reference, we write

$$
\widetilde{\psi}^{(-1 / 2)}=\Gamma_{L} \psi^{(-1 / 2)} \Gamma_{L}^{-1}, \quad \widetilde{B}=\Gamma_{L} B \Gamma_{R}, \quad \widetilde{\psi}^{(+1 / 2)}=\Gamma_{R}^{-1} \psi^{(+1 / 2)} \Gamma_{R} .
$$

Let us mention that these global gauge symmetries can be related to Kac-Moody algebras if we promote the gauge parameters to be chiral, see (50) below.

\subsection{Relativistic sector of the extended homogeneous hierarchy.}

In what follows we restrict the above construction to the subsystem associated to the flows $\left(t_{-1}, t_{-1 / 2}, t_{0}, t_{+1 / 2}, t_{+1}\right)$ in the extended homogeneous (also AKNS) hierarchy 5 . We have already identified the flows corresponding to $t_{0}$ with gauge symmetries and now we want to deduce the Lax operators we are going to use and to find some immediate consequences. In the next section we initiate the study of the fermionic symmetry flows associated to $t_{ \pm 1 / 2}$.

The relativistic sector of the extended homogeneous hierarchy is defined by (16), (17) for the two constant elements $\Lambda_{ \pm}^{( \pm 1)} \in \mathcal{K}_{ \pm}$of grades \pm 1 , associated to the isospectral times $t_{ \pm 1}=-x^{ \pm}$. We are interested in the action of (23), (24) as local gauge transformations preserving the compatibility conditions $\left[L_{+}, L_{-}\right]=\left[L_{+}^{\prime}, L_{-}^{\prime}\right]=0$ because of the relation

\footnotetext{
${ }^{5}$ The name AKNS is because for the loop algebra $\operatorname{sl}(2)^{(1)}$, the times $t_{+1}, t_{+2}$ leads to the AKNS equations while the times $t_{+1}, t_{-1}$ leads to the complex sine-Gordon equations (see [7]).
} 
of this integrable hierarchy with the Pohlmeyer reduced models. Then, we add two gauge connections $A_{ \pm}^{(L)} \in \mathfrak{h}_{L}$ and $A_{ \pm}^{(R)} \in \mathfrak{h}_{R}$ to the Lax operators and transforming as follows

$$
\widetilde{A}_{ \pm}^{(L)}=\Gamma_{L} A_{ \pm}^{(L)} \Gamma_{L}^{-1}+\partial_{ \pm} \Gamma_{L} \Gamma_{L}^{-1}, \quad \widetilde{A}_{ \pm}^{(R)}=\Gamma_{R}^{-1} A_{ \pm}^{(R)} \Gamma_{R}-\Gamma_{R}^{-1} \partial_{ \pm} \Gamma_{R}
$$

getting the desired covariant behavior $\widetilde{L}_{ \pm},=\Gamma_{L} L_{ \pm} \Gamma_{L}^{-1}$ and $\widetilde{L}_{ \pm}^{\prime}=\Gamma_{R}^{-1} L_{ \pm}^{\prime} \Gamma_{R}$. Explicitly, they are

$$
\begin{aligned}
& L_{+}=\partial_{+}-A_{+}^{(L)}+\left(A_{+}^{(0)}+Q_{+}^{(0)}+\psi_{+}^{(+1 / 2)}+\Lambda_{+}^{(+1)}\right), \\
& L_{-}=\partial_{-}-A_{-}^{(L)}-B\left(\psi_{-}^{(-1 / 2)}+\Lambda_{-}^{(-1)}\right) B^{-1}
\end{aligned}
$$

and

$$
\begin{aligned}
& L_{+}^{\prime}=\partial_{+}-A_{+}^{(R)}+B^{-1}\left(\psi_{+}^{(+1 / 2)}+\Lambda_{+}^{(+1)}\right) B, \\
& L_{-}^{\prime}=\partial_{-}-A_{-}^{(R)}-\left(A_{-}^{(0)}+Q_{-}^{(0)}+\psi_{-}^{(-1 / 2)}+\Lambda_{-}^{(-1)}\right),
\end{aligned}
$$

where

$\psi_{ \pm}^{( \pm 1 / 2)}= \pm\left[\psi^{(\mp 1 / 2)}, \Lambda_{ \pm}^{( \pm 1)}\right] \in \mathcal{M}_{F}^{( \pm 1 / 2)}, A_{ \pm}^{(0)}= \pm\left[\psi^{(\mp 1)}, \Lambda_{ \pm}^{( \pm 1)}\right] \in \mathcal{M}_{B}^{(0)}, Q_{ \pm}^{(0)}=\frac{1}{2}\left[\psi^{(\mp 1 / 2)},\left[\psi^{(\mp 1 / 2)}, \Lambda_{ \pm}^{( \pm 1)}\right]\right] \in \mathcal{K}_{B}^{(0)}$.

The dynamical fields are encoded in the expressions (26) but these relations are rather obscure, at least for the bosonic fields6. To identify the fields in a precise way we appeal to the relation (19) found above which relates the field content between $L_{ \pm}^{\prime}$ and $L_{ \pm}$. From $L_{ \pm}^{\prime}=B^{-1} L_{ \pm} B$ we find the relations

$$
A_{+}^{(L)}-A_{+}^{(0)}=\widehat{A}_{+}^{(L)}, \quad A_{-}^{(R)}+A_{-}^{(0)}=\widehat{A}_{-}^{(R)},
$$

where

$$
\widehat{A}_{+}^{(L)} \stackrel{\text { def }}{=} \partial_{+} B B^{-1}+B A_{+}^{(R)} B^{-1}+Q_{+}^{(0)}, \quad \widehat{A}_{-}^{(R)} \stackrel{\text { def }}{=}-B^{-1} \partial_{-} B+B^{-1} A_{-}^{(L)} B-Q_{-}^{(0)} .
$$

By projecting (28) along the gauge algebras $\mathfrak{h}_{L}, \mathfrak{h}_{R}$ we find the componets $A_{+}^{(L)}, A_{-}^{(R)}$ as functions of the other fields

$$
A_{+}^{(L)}=\mathcal{P}_{\mathfrak{h}_{L}}\left(\widehat{A}_{+}^{(L)}\right), \quad A_{-}^{(R)}=\mathcal{P}_{\mathfrak{h}_{R}}\left(\widehat{A}_{-}^{(R)}\right)
$$

and by projecting along the image subspace $\mathcal{M}$, we find the components $A_{ \pm}^{(0)}$ in terms of the Toda field $B$ and the gauge fields

$$
A_{+}^{(0)}=-\left(\partial_{+} B B^{-1}+B A_{+}^{(R)} B^{-1}\right)^{\|}, \quad A_{-}^{(0)}=-\left(B^{-1} \partial_{-} B-B^{-1} A_{-}^{(L)} B\right)^{\|} .
$$

Under the inner product (15) the relations (27) satisfy

$$
\left\langle\left(A_{+}^{(0)}\right)^{2}\right\rangle=\left\langle\left(\widehat{A}_{+}^{(L)}\right)^{2}-\left(A_{+}^{(L)}\right)^{2}\right\rangle, \quad\left\langle\left(A_{-}^{(0)}\right)^{2}\right\rangle=\left\langle\left(\widehat{A}_{-}^{(R)}\right)^{2}-\left(A_{-}^{(R)}\right)^{2}\right\rangle
$$

and are functionals of the physical fields in $\mathcal{M}$, as should be7.

Finally, we have the final form of the Lax pairs 8

$$
\begin{aligned}
& L_{+}(A)=\partial_{+}-\partial_{+} B B^{-1}-B A_{+}^{(R)} B^{-1}+\psi_{+}^{(+1 / 2)}+\Lambda_{+}^{(+1)}, \\
& L_{-}(A)=\partial_{-}-A_{-}^{(L)}-B\left(\psi_{-}^{(-1 / 2)}+\Lambda_{-}^{(-1)}\right) B^{-1}
\end{aligned}
$$

\footnotetext{
${ }^{6}$ Note that the fermions are automatically in the image subspace $\mathcal{M}=\widehat{\mathfrak{f}}^{\|}$. This is an important issue related to gauge fixing of the residual kappa symmetry in the reduction of superstring sigma models.

${ }^{7}$ These are contributions of the $T_{ \pm \pm}$components of the stress tensor $T_{\mu \nu}$, see (65) below.

${ }^{8}$ This is way the components $A_{+}^{(L)}, A_{-}^{(R)}$ were termed as "missing" in [8]. They do not appear explicitly in the final form of $L_{ \pm}$.
} 
and

$$
\begin{aligned}
& L_{+}^{\prime}(A)=\partial_{+}-A_{+}^{(R)}+B^{-1}\left(\psi_{+}^{(+1 / 2)}+\Lambda_{+}^{(+1)}\right) B \\
& L_{-}^{\prime}(A)=\partial_{-}+B^{-1} \partial_{-} B-B^{-1} A_{-}^{(L)} B-\psi_{-}^{(-1 / 2)}-\Lambda_{-}^{(-1)},
\end{aligned}
$$

with

$$
\widetilde{A}_{-}^{(L)}=\Gamma_{L} A_{-}^{(L)} \Gamma_{L}^{-1}+\partial_{-} \Gamma_{L} \Gamma_{L}^{-1}, \quad \widetilde{A}_{+}^{(R)}=\Gamma_{R}^{-1} A_{+}^{(R)} \Gamma_{R}-\Gamma_{R}^{-1} \partial_{+} \Gamma_{R}
$$

The equations of motion of the system are, by definition, given by the zero curvature $F_{+-}=\left[L_{+}, L_{-}\right]$of $(32)$ and they define the fermionic extension of the non-Abelian Toda models on the bi-quotient $H_{L} \backslash G / H_{R}$. They are given by

$$
\begin{aligned}
F_{+-}^{(+1 / 2)}= & -D_{-}^{(L)} \psi_{+}^{(+1 / 2)}+\left[B \psi_{-}^{(-1 / 2)} B^{-1}, \Lambda_{+}^{(+1)}\right] \\
F_{+-}^{(0)}= & D_{-}^{(L)}\left(\partial_{+} B B^{-1}+B A_{+}^{(R)} B^{-1}\right)-\partial_{+} A_{-}^{(L)}- \\
& -\left[\Lambda_{+}^{(+1)}, B \Lambda_{-}^{(-1)} B^{-1}\right]-\left[\psi_{+}^{(+1 / 2)}, B \psi_{-}^{(-1 / 2)} B^{-1}\right], \\
F_{+-}^{(-1 / 2)}= & B\left(-D_{+}^{(R)} \psi_{-}^{(-1 / 2)}+\left[\Lambda_{-}^{(-1)}, B^{-1} \psi_{+}^{(+1 / 2)} B\right]\right) B^{-1},
\end{aligned}
$$

where $D_{-}^{(L)}=\partial_{-}-\left[A_{-}^{(L)},\right]$ and $D_{+}^{(R)}=\partial_{+}-\left[A_{+}^{(R)},\right]$ are the covariant derivatives for the $H_{L} \times H_{R}$ actions of the gauge group. The equations given by (33) are simply $F_{+-}^{\prime}=B^{-1} F_{+-} B$.

The gauge fields $A_{ \pm}^{(L)}, A_{ \pm}^{(R)}$ are flat, an important property to be used later. To see this, we note that the grade zero equations of motion $F_{+-}^{(0)}$ and ${F_{+-}^{\prime(0)}}^{\prime}$ can be written, with the help of the Jacobi identity and the $F_{+-}^{( \pm 1 / 2)},{F_{+-}^{\prime}}^{\prime( \pm 1 / 2)}$ equations of motion, as

$$
\begin{aligned}
& F_{+-}^{(0)}=\partial_{-} \widehat{A}_{+}^{(L)}-\partial_{+} A_{-}^{(L)}+\left[\widehat{A}_{+}^{(L)}, A_{-}^{(L)}\right]-\left[\Lambda_{+}^{(+1)}, X_{-}^{(-1)}\right], \\
& F_{+-}^{\prime(0)}=\partial_{-} A_{+}^{(R)}-\partial_{+} \widehat{A}_{-}^{(R)}+\left[A_{+}^{(R)}, \widehat{A}_{-}^{(R)}\right]+\left[\Lambda_{-}^{(-1)}, X_{+}^{(+1)}\right],
\end{aligned}
$$

where $X_{ \pm}^{( \pm 1)} \in \widehat{\mathfrak{f}}$. Projecting $F_{+-}^{(0)}$ and $F_{+-}^{\prime(0)}$ along $\mathfrak{h}_{L}$ and $\mathfrak{h}_{R}$ respectively and taking into account (29), we conclude that the connections $A_{ \pm}^{(L)}, A_{ \pm}^{(R)}$ are pure gauge

$$
\left[\partial_{+}-A_{+}^{(L / R)}, \partial_{-}-A_{-}^{(L / R)}\right]=0 .
$$

In the (on-shell) gauge $A_{ \pm}^{(L / R)}=0$, the equations of motion of the fermionic extension of the non-abelian Toda models (35), together with the equations (29) become, respectively,

$$
\begin{aligned}
\partial_{-} \psi_{+}^{(+1 / 2)} & =\left[B \psi_{-}^{(-1 / 2)} B^{-1}, \Lambda_{+}^{(+1)}\right] \\
\partial_{-}\left(\partial_{+} B B^{-1}\right) & =\left[\Lambda_{+}^{(+1)}, B \Lambda_{-}^{(-1)} B^{-1}\right]+\left[\psi_{+}^{(+1 / 2)}, B \psi_{-}^{(-1 / 2)} B^{-1}\right], \\
\partial_{+} \psi_{-}^{(-1 / 2)} & =\left[\Lambda_{-}^{(-1)}, B^{-1} \psi_{+}^{(+1 / 2)} B,\right] \\
\left(\partial_{+} B B^{-1}+Q_{+}^{(0)}\right)^{\perp} & =\left(B^{-1} \partial_{-} B+Q_{-}^{(0)}\right)^{\perp}=0 .
\end{aligned}
$$

Note that in contrast to the purely bosonic non-Abelian Toda models which are characterized by the constraints $\left(\partial_{+} B B^{-1}\right)^{\perp}=\left(B^{-1} \partial_{-} B\right)^{\perp}=0$, the new constraints (38) are modified by the fermion bi-linears $Q_{ \pm}^{(0)}$. These constraints mean that there are no dynamical degrees of freedom associated to the kernel subalgebra $\mathcal{K}=\widehat{\mathfrak{f}}^{\perp}$, as expected. Note that they are also some sort of classical bosonization rules. 
To compute the classical spins of the fields, it is useful to add a central extension to the loop algebra (3) and introduce the central and gradation fields $\nu, \mu$, in order to restore the conformal invariance of the equations (37). Writing $B=\gamma \exp [\eta Q] \exp [\nu C], \gamma \in G$, we have that the equations

$$
\begin{aligned}
\partial_{-} \psi_{+}^{(+1 / 2)} & =e^{-\eta / 2}\left[\gamma \psi_{-}^{(-1 / 2)} \gamma^{-1}, \Lambda_{+}^{(+1)}\right] \\
\partial_{-}\left(\partial_{+} \gamma \gamma^{-1}+\gamma A_{+}^{(R)} \gamma^{-1}\right)-\partial_{+} A_{-}^{(L)}+\partial_{-} \partial_{+} C & =e^{-\eta}\left[\Lambda_{+}^{(+1)}, \gamma \Lambda_{-}^{(-1)} \gamma^{-1}\right]+e^{-\eta / 2}\left[\psi_{+}^{(+1 / 2)}, \gamma \psi_{-}^{(-1 / 2)} \gamma^{-1}\right] \\
\partial_{+} \psi_{-}^{(-1 / 2)} & =e^{-\eta / 2}\left[\Lambda_{-}^{(-1)}, \gamma^{-1} \psi_{+}^{(+1 / 2)} \gamma\right] \\
\partial_{-} \partial_{+} \eta Q & =0
\end{aligned}
$$

are invariant under conformal transformations $x^{+} \rightarrow \widetilde{x}^{+}=f\left(x^{+}\right), x^{-} \rightarrow \widetilde{x}^{-}=h\left(x^{-}\right)$, with the fields changing in the following manner

$$
\begin{aligned}
\widetilde{\gamma}\left(\widetilde{x}^{+}, \widetilde{x}^{-}\right) & =\gamma\left(x^{+}, x^{-}\right), \\
e^{-\widetilde{\eta}\left(\widetilde{x}^{+}, \widetilde{x}^{-}\right)} & =\left(f^{\prime}\right)^{-1}\left(h^{\prime}\right)^{-1} e^{-\eta\left(x^{+}, x^{-}\right)}, \\
e^{-\widetilde{\nu}\left(\widetilde{x}^{+}, \widetilde{x}^{-}\right)} & =\left(f^{\prime}\right)^{\delta}\left(h^{\prime}\right)^{\delta} e^{-\nu\left(x^{+}, x^{-}\right)}, \\
\widetilde{\psi}_{+}^{(+1 / 2)}\left(\widetilde{x}^{+}, \widetilde{x}^{-}\right) & =\left(f^{\prime}\right)^{-1 / 2} \psi_{+}^{(+1 / 2)}\left(x^{+}, x^{-}\right), \\
\widetilde{\psi}_{-}^{(-1 / 2)}\left(\widetilde{x}^{+}, \widetilde{x}^{-}\right) & =\left(h^{\prime}\right)^{-1 / 2} \psi_{-}^{(-1 / 2)}\left(x^{+}, x^{-}\right),
\end{aligned}
$$

where $\delta$ is arbitrary and $f^{\prime}=\partial_{+} f, h^{\prime}=\partial_{-} h$. Under a Lorentz transformation $x^{ \pm} \rightarrow \widetilde{x}^{ \pm}=\xi^{ \pm 1} x^{ \pm}$we can read off the classical spin of the fields. The bosonic fields are all scalars and the last two equations of (40) imply that

$$
\widetilde{\psi}^{( \pm 1 / 2)}\left(\xi x^{+}, \xi^{-1} x^{-}\right)=\xi^{ \pm 1 / 2} \psi^{( \pm 1 / 2)}\left(x^{+}, x^{-}\right)
$$

which instruct us to consider $\psi^{( \pm 1 / 2)}$ as legitimate two dimensional real spinors (Majorana-Weyl). This result is important because in the reduction process of superstring sigma models we start with world-sheet scalars but end up with world-sheet spinors. The reduction process will be review in chapter 3 .

To see how the Lax pair (32) change under Lorentz transformations we need to use $\widetilde{A}_{ \pm}\left(\xi x^{+}, \xi^{-1} x^{-}\right)=\xi^{\mp 1} A_{ \pm}\left(x^{+}, x^{-}\right)$. Then, we have

$$
\widetilde{L}_{ \pm}(A ; z)=\xi^{\mp 1} L_{ \pm}(A ; \xi z)
$$

and we see that the net effect of a Lorentz transformations is basically a rescaling of the spectral parameter $z \rightarrow \xi z$. From this we conclude that the equations of motion (35) are Lorentz invariant because they are $z$-independent. This result is also important in the context of reduced models because this means that equations of motion for the transverse degrees of freedom of the superstring are Lorentz invariant.

\subsection{Introducing the supersymmetry flows variations.}

In this section we try to find the supersymmetry transformations associated to $t_{ \pm 1 / 2}$ that leave invariant the fermionic non-Abelian Toda equations (35). The outcome, at this stage, is that we can defined consistent supersymmetry flows only when the gauge group $H_{L} \times H_{R}$ is global and there are no gauge fields $A=0$. A deeper study of these supersymmetric flows, also introduced in [9, will be presented elsewhere [10].

From the non-Abelian flow evolution equations (16), we can associate to the constant grassmanian elements $D^{( \pm 1 / 2)}=\epsilon_{i} F_{i}^{( \pm 1 / 2)} \in \mathcal{K}_{F}^{( \pm 1 / 2)}, i=1, \ldots, \operatorname{dim} \mathcal{K}_{F}^{( \pm 1 / 2)}$ of the fermionic kernel of grade $\pm 1 / 2$, i.e $\mathcal{K}_{F}^{( \pm 1 / 2)}$, the following two odd Lax variation operators

$$
L_{+1 / 2}=\delta_{+1 / 2}-D_{\Theta}^{(+1 / 2)}, \quad L_{-1 / 2}=\delta_{-1 / 2}+D_{\Pi}^{(-1 / 2)},
$$


where, as in (10), (11), we have

$$
D_{\Theta}^{(+1 / 2)}=\left(\Theta D^{(+1 / 2)} \Theta^{-1}\right)_{\geq 0}, \quad D_{\Pi}^{(-1 / 2)}=\left(\Pi D^{(-1 / 2)} \Pi^{-1}\right)_{\leq-1 / 2} .
$$

and a similar set of operators in the primed variables are obtained from (17). These satisfy the relation $L_{ \pm 1 / 2}^{\prime}=$ $B^{-1} L_{ \pm 1 / 2} B$ and imply that

$$
\delta_{+1 / 2} B B^{-1}=\left[\psi^{(-1 / 2)}+\theta^{(-1 / 2)}, D^{(+1 / 2)}\right], \quad B^{-1} \delta_{-1 / 2} B=-\left[\psi^{(+1 / 2)}+\theta^{(+1 / 2)}, D^{(-1 / 2)}\right] .
$$

At first sight, the supersymmetry variations for the fields would be extracted from the compatibility relations $\left[L_{ \pm 1 / 2}, L_{+}(A)\right]=\left[L_{ \pm 1 / 2}, L_{-}(A)\right]=0$ as was done in the case of the mKdV hierarchy [6] (see also [4]), i.e, when $A_{+}^{(R)}=$ $A_{-}^{(L)}=0$ in (32) with $\mathcal{K}_{B}^{(0)}=\varnothing$. Recall that under the local gauge transformations (23) the Lax operators transforms covariantly $\widetilde{L}_{ \pm}(A)=\Gamma_{L} L_{ \pm}(A) \Gamma_{L}^{-1}$. Then, in order for the compatibility relations $\left[L_{ \pm 1 / 2}, L_{+}(A)\right]=\left[L_{ \pm 1 / 2}, L_{-}(A)\right]=0$ to transform covariantly as well, we require the operators $L_{ \pm 1 / 2}$ to transform in the same way as $L_{ \pm}(A)$ do, i.e $\widetilde{L}_{ \pm 1 / 2}=$ $\Gamma_{L} L_{ \pm 1 / 2} \Gamma_{L}^{-1}$. Let's see when this can occur. Under $\widetilde{\Theta}=\Gamma_{L} \Theta \Gamma_{L}^{-1}$ and $\widetilde{\Pi}=\Gamma_{L} \Pi \Gamma_{R}$ we have

$$
D_{\widetilde{\Theta}}^{(+1 / 2)}=\Gamma_{L}\left(\Theta \Gamma_{L}^{-1} D^{(+1 / 2)} \Gamma_{L} \Theta^{-1}\right)_{\geq 0} \Gamma_{L}^{-1}, \quad D_{\widetilde{\Pi}}^{(-1 / 2)}=\Gamma_{L}\left(\Pi \Gamma_{R}^{-1} D^{(-1 / 2)} \Gamma_{R} \Pi^{-1}\right)_{\leq-1 / 2} \Gamma_{L}^{-1} .
$$

Then, the first obstruction for a covariant behavior appears when $\Gamma_{L}^{-1} D^{(+1 / 2)} \Gamma_{L} \neq D^{(+1 / 2)}$ and $\Gamma_{R}^{-1} D^{(-1 / 2)} \Gamma_{R} \neq$ $D^{(-1 / 2)}$. Now, if we assume that there are elements of $\mathcal{K}_{F}^{( \pm 1 / 2)}$ commuting with the entire gauge algebras $\mathfrak{h}_{L}$ and $\mathfrak{h}_{R}$ and that $\Gamma_{L}$ is invariant under the $\delta_{ \pm 1 / 2}$ flows i.e $\delta_{ \pm 1 / 2} \Gamma_{L} \Gamma_{L}^{-1}=0$, then the desired gauge transformation holds. If this is the case we have from $\left[L_{ \pm 1 / 2}, L_{+}(A)\right]=\left[L_{ \pm 1 / 2}, L_{-}(A)\right]=0$, the following "supersymmetry" transformation 9

$$
\begin{aligned}
\left(\delta_{+1 / 2} B B^{-1}\right)^{\|} & =\left[\psi^{(-1 / 2)}, D^{(+1 / 2)}\right], \\
\delta_{+1 / 2} \psi_{+}^{(+1 / 2)} & =\left[\left(\left(D_{+}^{(R)} B\right) B^{-1}\right)^{\|}, D^{(+1 / 2)}\right]+\left[\theta_{+}^{(0)}, \psi_{+}^{(+1 / 2)}\right], \\
\delta_{+1 / 2} \psi_{-}^{(-1 / 2)} & =-\left[\Lambda_{-}^{(-1)}, B^{-1} D^{(+1 / 2)} B\right], \\
\delta_{+1 / 2} A_{-}^{(L)} & =-\left[\left(B \psi_{-}^{(-1 / 2)} B^{-1}\right)^{\perp}, D^{(+1 / 2)}\right]+D_{-}^{(L)}\left(\theta_{+}^{(0)}\right), \\
\delta_{+1 / 2} A_{+}^{(R)} & =0,
\end{aligned}
$$

and

$$
\begin{aligned}
\left(B^{-1} \delta_{-1 / 2} B\right)^{\|} & =-\left[\psi^{(+1 / 2)}, D^{(-1 / 2)}\right], \\
\delta_{-1 / 2} \psi_{+}^{(+1 / 2)} & =\left[\Lambda_{+}^{(+1)}, B D^{(-1 / 2)} B^{-1}\right], \\
\delta_{-1 / 2} \psi_{-}^{(-1 / 2)} & =-\left[\left(B^{-1} D_{-}^{(L)} B\right)^{\|}, D^{(-1 / 2)}\right]+\left[\theta_{-}^{(0)}, \psi_{-}^{(-1 / 2)}\right], \\
\delta_{-1 / 2} A_{+}^{(R)} & =-\left[\left(B^{-1} \psi_{+}^{(+1 / 2)} B\right)^{\perp}, D^{(-1 / 2)}\right]+D_{+}^{(R)}\left(\theta_{-}^{(0)}\right), \\
\delta_{+1 / 2} A_{-}^{(L)} & =0,
\end{aligned}
$$

where $\theta_{ \pm}^{(0)}=\left[\theta^{(\mp 1 / 2)}, D^{( \pm 1 / 2)}\right]$, we have used the equations of motion (35) and the important assumption that $\left[D^{(+1 / 2)}, A_{-}^{(L)}\right]=\left[D^{(-1 / 2)}, A_{+}^{(R)}\right]=0$.

\footnotetext{
${ }^{9}$ These transformations when $\theta_{ \pm}^{(0)}=0$, are essentially the same as the ones proposed by hand in [1] to be the on-shell supersymmetry transformations of a Lagrangian formulation of (35) in the particular case of the Polhmeyer reduction of the $A d S_{5} \times S^{5}$ superstring sigma model.
} 
The assumption that an element $D \in \mathcal{K}_{F}$ in the fermionic kernel $\mathcal{K}_{F}$ commute with the entire gauge algebra is too stringent because the odd part $\mathfrak{g}_{1} \in \mathfrak{g}$ of a given Lie superalgebra $\mathfrak{g}=\mathfrak{g}_{0} \oplus \mathfrak{g}_{1}$ provides the carrier space for some faithful representation $R$ of the even part $\mathfrak{g}_{0} \in \mathfrak{g}$ i.e $\left[\mathfrak{g}_{0}, \mathfrak{g}_{1}\right]=R\left(\mathfrak{g}_{0}\right) \mathfrak{g}_{1}$. Then, although tempting, the transformations (45), (46) are incorrect in origin and the method used with success in the extended mKdV hierarchy does not apply here in the extended homogeneous hierarchy anymore. Another problem is related to the local character of $\Gamma_{L}$ and $\Gamma_{R}$. After a gauge transformation, the supersymmetry parameters $\widetilde{D}^{(+1 / 2)}=\Gamma_{L}^{-1} D^{(+1 / 2)} \Gamma_{L}$ and $\widetilde{D}^{(-1 / 2)}=\Gamma_{R}^{-1} D^{(-1 / 2)} \Gamma_{R}$ are not constants and in principle there is no consistent supersymmetry. However, consistent supersymmetry flows can be defined when the gauge group is global because the action of $\mathfrak{h}_{L} \times \mathfrak{h}_{R}$ preserves the fermionic kernel $\mathcal{K}_{F}^{( \pm 1 / 2)}$. They simply rotate the generators $F_{i}^{( \pm 1 / 2)}$, say $\left[K_{L}^{(0)}, F_{i}^{(+1 / 2)}\right]=R\left(K_{L}^{(0)}\right)_{i j} F_{j}^{(+1 / 2)}$, which is equivalent to a linear combination of the constant grasmannian parameters $\epsilon_{i}$ and $\bar{\epsilon}_{j}$. Then, we see that the global supersymmetry we are dealing with is of the extended type, namely, they include several fermionic symmetry flows transforming under the gauge algebra. This should not come as a surprise because the kernel algebra $\mathcal{K}=\mathcal{K}_{B} \oplus \mathcal{K}_{F}$ is a sub-superalgebra of $\widehat{\mathfrak{f}}=\mathcal{K} \oplus \mathcal{M}$ anyway, in which the symmetries $\delta_{\mathcal{K}}$ are generated by $\mathcal{K}$ through the flow equations (16), (17). Below, we will use the Drinfeld-Sokolov procedure to construct a set of $\operatorname{dim} \mathcal{K}_{F}^{( \pm 1 / 2)}$ non-local fermionic conserved charges associated to the flows $\delta_{ \pm 1 / 2}$ and transforming under the global part of the gauge group, arriving to a meaningful result, see (668).

In the on-shell gauge $A_{-}^{(L)}=A_{+}^{(R)}=0$ with global gauge group, the supersymmetry transformations of the extended homogeneous hierarchy are given by (45), (46) (with $A_{-}^{(L)}=A_{+}^{(R)}=0$ ) and this fix the forms of the non-local terms $\theta^{( \pm 1 / 2)}$ to be

$$
\theta^{( \pm 1 / 2)}=\partial_{ \pm}^{-1}\left(B^{\mp 1} \psi_{ \pm}^{( \pm 1 / 2)} B^{ \pm 1}\right)^{\perp}
$$

implying also the invariance of the constraints $A_{+}^{(L)}=A_{-}^{(R)}=0$, i.e (38) under the $\delta_{ \pm 1 / 2}$ flows. Below, we will show that these supersymmetries are Hamiltonian flows under the second Poisson structure of the extended homogeneous hierarchy, see (93).

\subsection{Lagrangian formulation of the semi-symmetric space sine-Gordon models.}

Now that we have identified the dynamical fields, we want to introduce the action functional which have the fermionic Toda equations (35) as Euler-Lagrange equations of motion. Below, we shall see that this is the action functional behind the Pohlmeyer reduction process.

Notation 1 The light-cone notation used for the flat Minkowski space $\Sigma$ is $x^{ \pm}=\frac{1}{2}\left(x^{0} \pm x^{1}\right), \partial_{ \pm}=\partial_{0} \pm \partial_{1}, \eta_{+-}=$ $\eta_{-+}=2, \eta^{+-}=\eta^{-+}=\frac{1}{2}, \epsilon_{+-}=-\epsilon_{-+}=2, \epsilon^{-+}=-\epsilon^{+-}=\frac{1}{2}$ corresponding to the metric $\eta_{00}=1, \eta_{11}=-1$ and antisymmetric symbol $\epsilon_{10}=-\epsilon_{01}=+1$. A mass scale is introduced by setting $\Lambda_{ \pm}^{( \pm 1)} \rightarrow \mu \Lambda_{ \pm}^{( \pm 1)}$ and $\psi^{( \pm 1 / 2)} \rightarrow$ $\mu^{-1 / 2} \psi^{( \pm 1 / 2)}$.

Consider the action functional

$$
\begin{aligned}
S_{\text {Toda }}[B, \psi]= & S_{W Z N W}[B]-\frac{k}{4 \pi} \int_{\Sigma}\left\langle\psi_{+}^{(+1 / 2)} \partial_{-} \psi^{(-1 / 2)}+\psi_{-}^{(-1 / 2)} \partial_{+} \psi^{(+1 / 2)}\right\rangle+ \\
& +\frac{k}{2 \pi} \int_{\Sigma}\left\langle\Lambda_{+}^{(+1)} B \Lambda_{-}^{(-1)} B^{-1}+\psi_{+}^{(+1 / 2)} B \psi_{-}^{(-1 / 2)} B^{-1}\right\rangle, \\
S_{W Z N W}[B]= & -\frac{k}{4 \pi}\left(\int_{\Sigma}\left\langle B^{-1} \partial_{+} B B^{-1} \partial_{-} B\right\rangle-\frac{1}{3} \int_{M}\left\langle\left(B^{-1} d B\right)^{3}\right\rangle\right),
\end{aligned}
$$

which is the action deduced in [5] to describe the supersymmetric sector of the extended super mKdV hierarchy. 
In the present situation, i.e in the extended homogeneous hierarchy, this action is invariant under the global gauge $H_{L} \times H_{R}$ group transformations (24). Moreover, it is also invariant under the Kac-Moody-type transformations

$$
\begin{aligned}
\widetilde{B} & =\Gamma_{L}\left(x^{+}\right) B \Gamma_{R}\left(x^{-}\right), \\
\widetilde{\psi}^{(-1 / 2)} & =\Gamma_{L}\left(x^{+}\right) \psi^{(-1 / 2)} \Gamma_{L}^{-1}\left(x^{+}\right), \\
\widetilde{\psi}^{(+1 / 2)} & =\Gamma_{R}^{-1}\left(x^{-}\right) \psi^{(+1 / 2)} \Gamma_{R}\left(x^{-}\right)
\end{aligned}
$$

as can be seen with the help of the Polyakov-Wiegmann identity 10.

Taking $\Gamma_{L}\left(x^{+}\right)=\exp \omega_{L}\left(x^{+}\right)$and $\Gamma_{R}\left(x^{-}\right)=\exp \omega_{R}\left(x^{+}\right)$we find the transformations

$$
\delta \psi^{(-1 / 2)}=\left[\omega_{L}\left(x^{+}\right), \psi^{(-1 / 2)}\right], \quad \delta B B^{-1}=\omega_{L}\left(x^{+}\right)+B \omega_{R}\left(x^{-}\right) B^{-1}, \quad \delta \psi^{(+1 / 2)}=-\left[\omega_{R}\left(x^{-}\right), \psi^{(+1 / 2)}\right],
$$

allowing to compute the variation of (48). It is given by

$$
\frac{2 \pi}{k} \delta S_{\text {Toda }}[B, \psi]=\int\left\langle\omega_{L}\left(x^{+}\right) \partial_{-}\left(\partial_{+} B B^{-1}+Q_{+}^{(0)}\right)+\omega_{R}\left(x^{-}\right) \partial_{+}\left(B^{-1} \partial_{-} B+Q_{-}^{(0)}\right)\right\rangle,
$$

where we have used the jacobi identity, the ad-invariance of the inner product and $\left[\Lambda_{ \pm}^{( \pm 1)}, \mathcal{K}_{B}^{(0)}\right]=0$. This implies the existence of chiral currents $\partial_{-} J_{+}\left(x^{+}\right)=\partial_{+} J_{-}\left(x^{-}\right)=0$, where (cf. (38))

$$
J_{+}\left(x^{+}\right)=\mathcal{P}_{\mathfrak{h}_{L}}\left(\partial_{+} B B^{-1}+Q_{+}^{(0)}\right), \quad J_{-}\left(x^{-}\right)=\mathcal{P}_{\mathfrak{h}_{R}}\left(B^{-1} \partial_{-} B+Q_{-}^{(0)}\right) .
$$

The action is also invariant under fermionic shifts

$$
\delta \psi^{( \pm 1 / 2)}=\theta^{( \pm 1 / 2)}\left(x^{\mp}\right),
$$

where $\theta^{( \pm 1 / 2)} \in \mathcal{K}_{F}^{( \pm 1 / 2)}$ and $\delta B B^{-1}=0$ leading to the following variation

$$
\frac{4 \pi}{k} \delta S_{\text {Toda }}[B, \psi]=\int\left\langle\theta^{(+1 / 2)}\left(x^{-}\right) \partial_{+} \psi_{-}^{(-1 / 2)}+\theta^{(-1 / 2)}\left(x^{+}\right) \partial_{-} \psi_{+}^{(+1 / 2)}\right\rangle .
$$

Note the strong resemblance with the super Kac-Moody currents obtained from a supersymmetric WZNW model [11. However, their origin are quite different as fermions in a supersymmetric WZNW model parametrize the same Lie algebra as the bosons while in the action (48) they parametrize the odd subspace of a Lie superalgebra i.e bosons and fermions are in different subspaces. This difference is very important in our approach to supersymmetry flows and this will be discussed below when we study the supersymmetry properties of the Pohlmeyer reduction of superstring sigma models. Clearly, the invariance under (50), (51) and its current algebra deserves a deeper study.

At this point we can identify the fermionic extension of the non-Abelian Toda models as the Hamiltonian reduction of the phase space of (48) defined by the vanishing of the Kac-Moody-type currents $J_{ \pm}\left(x^{ \pm}\right)=0$, which are exactly the constraints given by (38). Then, to obtain a Lagrangian formulation for the equations (37) we need to impose the constraints $J_{ \pm}\left(x^{ \pm}\right)=0$ off-shell and this can be done by considering a local gauge group $H_{L} \times H_{R}$ and introducing gauge fields.

\footnotetext{
${ }^{10}$ This is given by$$
S_{W Z N W}[A B C]=S_{W Z N W}[A]+S_{W Z N W}[B]+S_{W Z N W}[C]-
$$$$
-\frac{k}{2 \pi} \int\left\langle\left(A^{-1} \partial_{-} A\right)\left(\partial_{+} B B^{-1}\right)+\left(B^{-1} \partial_{-} B\right)\left(\partial_{+} C C^{-1}\right)+\left(A^{-1} \partial_{-} A\right) B\left(\partial_{+} C C^{-1}\right) B^{-1}\right\rangle \text {. }
$$ 
The Lagrangian formulation of the system (37), (38) exists, as explained in [12] (see also [13] for the original formulation), only when the gauge groups $H_{L}, H_{R}$ are isomorphic to some Lie group $H=\exp \mathfrak{h}$ with Lie algebra $\mathfrak{h}$ such that $H_{L}=\epsilon_{L}(H), H_{R}=\epsilon_{R}(H)$, where $\epsilon_{L}, \epsilon_{R}: H \rightarrow G$ are two group homomorphisms that descend to embeddings of the corresponding Lie algebras and that satisfy the anomaly free condition

$$
\left\langle\epsilon_{L}(a) \epsilon_{L}(b)\right\rangle-\left\langle\epsilon_{R}(a) \epsilon_{R}(b)\right\rangle=0, \quad \forall a, b \in \mathfrak{h} .
$$

In this case the gauge group is reduced 11 from $H_{L} \times H_{R}$ to a diagonal subgroup of $H \subset H_{L} \times H_{R}$ and the Lagrangian is simply given by an appropriate covariantization of (48) defining now the semi-symmetric space sine-Gordon model (SSSSG) action

$$
\begin{aligned}
S_{S S S S G}[B, \psi]= & S_{g W Z N W}[B, A]_{G / H}-\frac{k}{4 \pi} \int_{\Sigma}\left\langle\psi_{+}^{(+1 / 2)} D_{-}^{(L)} \psi^{(-1 / 2)}+\psi_{-}^{(-1 / 2)} D_{+}^{(R)} \psi^{(+1 / 2)}\right\rangle+ \\
& +\frac{k}{2 \pi} \int_{\Sigma}\left\langle\Lambda_{+}^{(+1)} B \Lambda_{-}^{(-1)} B^{-1}+\psi_{+}^{(+1 / 2)} B \psi_{-}^{(-1 / 2)} B^{-1}\right\rangle,
\end{aligned}
$$

where $D_{-}^{(L)}=\partial_{-}-\left[\epsilon_{L}\left(A_{-}\right),\right], D_{+}^{(R)}=\partial_{+}-\left[\epsilon_{R}\left(A_{+}\right),\right]$are the covariant derivatives for the action of the gauge group $H, A_{ \pm} \in \mathfrak{h}$ is the gauge field and

$$
S_{g W Z N W}[B, A]_{G / H}=S_{W Z N W}[B]_{G}-\frac{k}{2 \pi} \int_{\Sigma}\left\langle\begin{array}{c}
-\epsilon_{L}\left(A_{-}\right) \partial_{+} B B^{-1}+\epsilon_{R}\left(A_{+}\right) B^{-1} \partial_{-} B- \\
-\epsilon_{L}\left(A_{-}\right) B \epsilon_{R}\left(A_{+}\right) B^{-1}+\epsilon_{L}\left(A_{+}\right) \epsilon_{L}\left(A_{-}\right)
\end{array}\right\rangle
$$

is the standard gauged WZNW action. The action (52) is invariant under the following gauge transformations

$$
\begin{aligned}
\widetilde{B} & =\epsilon_{L}(\Gamma) B \epsilon_{R}\left(\Gamma^{-1}\right), \\
\widetilde{\psi}^{(-1 / 2)} & =\epsilon_{L}(\Gamma) \psi^{(-1 / 2)} \epsilon_{L}\left(\Gamma^{-1}\right), \\
\widetilde{\psi}^{(+1 / 2)} & =\epsilon_{R}(\Gamma) \psi^{(+1 / 2)} \epsilon_{R}\left(\Gamma^{-1}\right), \\
\widetilde{A}_{ \pm} & =\Gamma A_{ \pm} \Gamma^{-1}+\partial_{ \pm} \Gamma \Gamma^{-1},
\end{aligned}
$$

where $\Gamma_{L}=\epsilon_{L}(\Gamma)$ and $\Gamma_{R}=\epsilon_{R}\left(\Gamma^{-1}\right)$ in (50) are local elements and we have used (34) in the last line.

An arbitrary variation of (52) is given by

$$
\begin{aligned}
\frac{2 \pi}{k} \delta S_{S S S S G}[B, \psi]= & \int_{\Sigma}\left\langle\left(\delta B B^{-1}-B \delta \psi^{(+1 / 2)} B^{-1}-\delta \psi^{(-1 / 2)}\right) F_{+-}\right\rangle+ \\
& +\int_{\Sigma}\left\langle\delta A_{-}^{(L)}\left\{-A_{+}^{(L)}+\partial_{+} B B^{-1}+B A_{+}^{(R)} B^{-1}+Q_{+}^{(0)}\right\}\right\rangle+ \\
& +\int_{\Sigma}\left\langle\delta A_{+}^{(R)}\left\{-A_{-}^{(R)}-B^{-1} \partial_{-} B+B^{-1} A_{-}^{(L)} B-Q_{-}^{(0)}\right\}\right\rangle,
\end{aligned}
$$

where the curvature components $F_{+-}^{(0)}$ and $F_{+-}^{( \pm 1 / 2)}$ are given in (35) but now with $A_{-}^{(L)}=\epsilon_{L}\left(A_{-}\right)$and $A_{+}^{(R)}=\epsilon_{R}\left(A_{+}\right)$. Then, the Lax pair associated to the action (52) is simply the reduction of (32), i.e

$$
\begin{aligned}
& L_{+}(A)=\partial_{+}-\partial_{+} B B^{-1}-B \epsilon_{R}\left(A_{+}\right) B^{-1}+\psi_{+}^{(+1 / 2)}+\Lambda_{+}^{(+1)}, \\
& L_{-}(A)=\partial_{-}-\epsilon_{L}\left(A_{-}\right)-B\left(\psi_{-}^{(-1 / 2)}+\Lambda_{-}^{(-1)}\right) B^{-1},
\end{aligned}
$$

supplemented by the constraints given by the $A_{ \pm}$equations of motion. It is not difficult to see that the last two equations of motion in (54) and the definitions of the missing components (29) are the same in this case, and that the two equations (36) reduce to the flatness of the only gauge field involved $A_{ \pm}$

$$
\left[\partial_{+}-A_{+}, \partial_{-}-A_{-}\right]=0,
$$

\footnotetext{
${ }^{11}$ This reduction can also be seen as a partially gauge fixing of the $H_{L} \times H_{R}$ gauge symmetry [1].
} 
which enables the on-shell gauge $A_{+}=A_{-}=0$, as in the last line of (37).

It is important to mention that the $A_{ \pm}$equations of motion can also be interpreted as a partial gauge fixing of the $H_{L} \times H_{R}$ gauge symmetry in the definitions (29), see [1] for the details.

\subsection{The Drinfeld-Sokolov procedure: the $\operatorname{dim} \mathcal{K}_{F}^{( \pm 1 / 2)} 2 \mathrm{D}$ spin $\pm 1 / 2$ supercharges.}

In section 2.3 we have found some difficulties in finding the supersymmetry flow variations for the fields due to the local character of the gauge group $H_{L} \times H_{R}$. However, a consistent set of transformations were singled out and we shall return to them later in section 2.7. Here we focus on the construction of the fermionic conserved charges associated to the symmetry flows $t_{ \pm 1 / 2}$ in the general situation when the gauge group $H_{L} \times H_{R}$ is local.

One of the advantages of the dressing approach adopted above, is that we can apply the Drinfeld-Sokolov (DS) procedure and viceversa. It provides a systematic method for constructing the local and non-local conserved charges associated to the symmetry flows (16), (17). Inspired by a similar computation done in [8, here we will extract the vector, spinor and tensor conserved currents associated to the sub-sector $\left(t_{0}, t_{ \pm 1 / 2}, t_{ \pm 1}\right)$.

From (16), (17) but now in the presence of gauge fields, we have the following dressing relations

$$
\begin{array}{ll}
L_{+}(A)=\Theta\left(\partial_{+}+\Lambda_{+}^{(+1)}\right) \Theta^{-1}, & L_{-}(A)=\Theta\left(\partial_{-}\right) \Theta^{-1}, \\
L_{+}^{\prime}(A)=\Pi^{\prime}\left(\partial_{+}\right) \Pi^{\prime-1}, & L_{-}^{\prime}(A)=\Pi^{\prime}\left(\partial_{-}-\Lambda_{-}^{(-1)}\right) \Pi^{\prime-1},
\end{array}
$$

associated to the Lax operators (32), (33).

The dressing matrix $\Theta$ factorizes as $\Theta=U_{-} S_{-}$, where $U_{-} \in \exp u_{-}, u_{-}=\oplus_{r \leq-1 / 2} \widehat{\mathfrak{f}}_{r}^{\|} \subset \mathcal{M}$ is a local functional of the fields and $S_{-} \in \exp s_{-}, s_{-}=\oplus_{r \leq-1 / 2} \widehat{\mathfrak{f}}_{r}^{\perp} \subset \mathcal{K}$ is a non-local functional of the fields, splitting the dressing of the vacuum Lax operators, i.e $L_{ \pm}=\Theta L_{ \pm}^{V} \Theta^{-1}$, as a two step process [14. An $U_{-}$and an $S_{-}$rotation given respectively by

$$
\begin{array}{rlrl}
U_{-}^{-1} L_{+}(A) U_{-} & =\partial_{+}+\Lambda_{+}^{(+1)}+K_{+}^{(-)}, & U_{-}^{-1} L_{-}(A) U_{-}=\partial_{-}+K_{-}^{(-)} \\
\partial_{+}+\Lambda_{+}^{(+1)}+K_{+}^{(-)} & =S_{-}\left(\partial_{+}+\Lambda_{+}^{(+1)}\right) S_{-}^{-1}, & \partial_{-}+K_{-}^{(-)} & =S_{-}\left(\partial_{-}\right) S_{-}^{-1}
\end{array}
$$

where $K_{ \pm}^{(-)}=\Sigma_{r \leq 0} K_{ \pm}^{(r)}, K_{ \pm}^{(r)} \in \mathcal{K}$ are the conserved current components we want to find.

Similarly, for the second line in (56) we have $\Pi^{\prime}=U_{+} S_{+}$, where $U_{+} \in \exp u_{+}, u_{+}=\oplus_{r \geq+1 / 2} \widehat{\mathfrak{f}}_{r}^{\|} \subset \mathcal{M}$ is local and $S_{+} \in \exp s_{+}, s_{+}=\oplus_{r \geq+1 / 2} \widehat{\mathfrak{f}}_{r}^{\perp} \subset \mathcal{K}$ is non-local in the fields. Then, we have

$$
\begin{aligned}
U_{+}^{-1} L_{+}^{\prime}(A) U_{+} & =\partial_{+}+K_{+}^{(+)}, & U_{+}^{-1} L_{-}^{\prime}(A) U_{+} & =\partial_{-}-\Lambda_{-}^{(-1)}+K_{-}^{(+)}, \\
\partial_{+}+K_{+}^{(+)} & =S_{+}\left(\partial_{+}\right) S_{+}^{-1}, & \partial_{-}-\Lambda_{-}^{(-1)}+K_{-}^{(+)} & =S_{-}\left(\partial_{-}-\Lambda_{-}^{(-1)}\right) S_{-}^{-1},
\end{aligned}
$$

where $K_{ \pm}^{(+)}=\Sigma_{r \geq 0} K_{ \pm}^{(r)}, K_{ \pm}^{(r)} \in \mathcal{K}$.

The conservation laws are extracted by projecting the zero curvature conditions of (57) and (59) along the kernel subspace $\mathcal{K}$ grade by grade. This is roughly speaking, the Drinfeld-Sokolov procedure. Of course, it provides local conservation laws for all the isospectral flows $t_{ \pm n}$.

It is important to mention that (57) and (59) are in canonical form, in the sense that $\Theta=U_{-} S_{-}$and $\Pi^{\prime}=U_{+} S_{+}$ are splitted as local and non-local pieces allowing to obtain $K_{ \pm}^{(-)}, K_{ \pm}^{(+)}$as functionals of the components of $U_{ \pm}$only. However, these relations are subject to an ambiguity induced by the gauge transformations $U_{ \pm} \rightarrow U_{ \pm} \widetilde{S}_{ \pm}$with $\widetilde{S}_{ \pm}$ parametrized in the same way as $S_{ \pm}$. This action does not change the LHS of (57) and (59) but changes the RHS side. 
For example, for (57) we have

$$
U_{-}^{-1} L_{+}(A) U_{-}=\widetilde{S}_{-}\left(\partial_{+}+\Lambda_{+}^{(+1)}+\widetilde{K}_{+}^{(-)}\right) \widetilde{S}_{-}^{-1}, \quad U_{-}^{-1} L_{-}(A) U_{-}=\widetilde{S}_{-}\left(\partial_{-}+\widetilde{K}_{-}^{(-)}\right) \widetilde{S}_{-}^{-1}
$$

and a similar expression for (59) leading to a non-local gauge transformation between the current components. This observation will be useful below because allow for an explanation of an apparent discrepancy between the non-local supersymmetry flow variations as presented above in (45), (46) in the gauge $A^{(L / R)}=0$ and the local supersymmetry flow variations induced by the canonical form of the supercharges extracted from (57), (59) in the gauge $A^{(L / R)}=0$. See (69) and proposition 4 below.

The components of grades $0,-1 / 2$ and -1 of the first equation of (57) along the kernel $\mathcal{K}$, are given by

$$
\begin{aligned}
0 & : \quad K_{+}^{(0)}=-A_{+}^{(L)}, \\
-1 / 2 & : \quad K_{+}^{(-1 / 2)}=\left[\psi^{(-1 / 2)}, \widehat{A}_{+}^{(L)}\right]^{\perp}, \\
-1 & :\left\langle\Lambda_{+}^{(+1)}, K_{+}^{(-1)}\right\rangle=+\frac{1}{2}\left\langle\left(\widehat{A}_{+}^{(L)}\right)^{2}-\left(A_{+}^{(L)}\right)^{2}\right\rangle+\frac{1}{2}\left\langle\psi_{+}^{(+1 / 2)} D_{+}^{(L)} \psi^{(-1 / 2)}\right\rangle+\left\langle\left(Q_{+}^{(0)}\right)^{2}\right\rangle,
\end{aligned}
$$

where we have taken $u^{(-1 / 2)}=\psi^{(-1 / 2)}, u^{(-1)}=\psi^{(-1)}$ and used the definitions (29) and (26). In the last relation we projected along $\Lambda_{+}^{(+1)}$ in order to simplify expressions. The components of grades $0,-1 / 2$ and -1 of the second equation of (57) along the kernel $\mathcal{K}$, are given by

$$
\begin{array}{rll}
0 & : \quad K_{-}^{(0)}=-A_{-}^{(L)} \\
-1 / 2 & : \quad K_{-}^{(-1 / 2)}=-\left(B \psi_{-}^{(-1 / 2)} B^{-1}\right)^{\perp}, \\
-1 & : \quad\left\langle\Lambda_{+}^{(+1)}, K_{-}^{(-1)}\right\rangle=-\left\langle\Lambda_{+}^{(+1)} B \Lambda_{-}^{(-1)} B^{-1}+\frac{1}{2} \psi_{+}^{(+1 / 2)} B \psi_{-}^{(-1 / 2)} B^{-1}\right\rangle,
\end{array}
$$

where in the last relation we have used the equations of motion for $\psi^{(-1 / 2)}$.

In a complete analogous way, we have for the first equation of (59) along the kernel subspaces of grades $0,+1 / 2$ and +1 , the following components

$$
\begin{array}{rll}
0 & : K_{+}^{\prime(0)}=-A_{+}^{(R)} \\
+1 / 2 & : K_{+}^{(+1 / 2)}=\left(B^{-1} \psi_{+}^{(+1 / 2)} B\right)^{\perp} \\
+1: & \left\langle\Lambda_{-}^{(-1)}, K_{+}^{(+1)}\right\rangle=-\left\langle\Lambda_{+}^{(+1)} K_{-}^{(-1)}\right\rangle,
\end{array}
$$

where $u^{(+1 / 2)}=-\psi^{(+1 / 2)}$ and $u^{(+1)}=-\psi^{(+1)}$. The second equation of (59) provides

$$
\begin{array}{rll}
0 & : \quad K_{-}^{\prime(0)}=-A_{-}^{(R)} \\
+1 / 2 & : \quad K_{-}^{(+1 / 2)}=-\left[\psi^{(+1 / 2)}, \widehat{A}_{-}^{(R)}\right]^{\perp}, \\
+1 & :\left\langle\Lambda_{-}^{(-1)}, K_{-}^{(+1)}\right\rangle=-\frac{1}{2}\left\langle\left(\widehat{A}_{-}^{(R)}\right)^{2}-\left(A_{-}^{(R)}\right)^{2}\right\rangle-\frac{1}{2}\left\langle\psi_{-}^{(-1 / 2)} D_{-}^{(R)} \psi^{(+1 / 2)}\right\rangle-\left\langle\left(Q_{-}^{(0)}\right)^{2}\right\rangle .
\end{array}
$$

The zero curvatures of the LHS of (57) and (59) imply 12

$$
\begin{aligned}
& \partial_{+} K_{-}^{(-)}-\partial_{-} K_{+}^{(-)}+\left[K_{+}^{(-)}, K_{-}^{(-)}\right]=0 \\
& \partial_{+} K_{-}^{(+)}-\partial_{-} K_{+}^{(+)}+\left[K_{+}^{(+)}, K_{-}^{(+)}\right]=0 .
\end{aligned}
$$

\footnotetext{
${ }^{12}$ The advantage of the DS expressions (62) is that the process of getting conserved charges can be continued infinitely without invoking an action functional and without reducing the gauge symmetry to a diagonal subgroup of $H_{L} \times H_{R}$ as required by a Lagrangian formulation.
} 
Decomposing the first equation of (62) in terms of $0,-1 / 2$ and -1 grades we get

$$
\begin{array}{rll}
0: & {\left[\partial_{+}-A_{+}^{(L)}, \partial_{-}-A_{-}^{(L)}\right]=0} \\
-1 / 2: & D_{+}^{(L)} K_{-}^{(-1 / 2)}-D_{-}^{(L)} K_{+}^{(-1 / 2)}=0 \\
-1: & \partial_{-}\left(\left\langle\Lambda_{+}^{(+1)}, K_{+}^{(-1)}\right\rangle\right)+\partial_{+}\left(-\left\langle\Lambda_{+}^{(+1)}, K_{-}^{(-1)}\right\rangle\right)=0 .
\end{array}
$$

Similarly, the second equation of (62) in terms of $0,+1 / 2$ and +1 grades gives

$$
\begin{aligned}
0: & {\left[\partial_{+}-A_{+}^{(R)}, \partial_{-}-A_{-}^{(R)}\right]=0 } \\
+1 / 2: & D_{+}^{(R)} K_{-}^{(+1 / 2)}-D_{-}^{(R)} K_{+}^{(+1 / 2)}=0, \\
+1: & \partial_{-}\left(\left\langle\Lambda_{-}^{(-1)}, K_{+}^{(+1)}\right\rangle\right)+\partial_{+}\left(-\left\langle\Lambda_{-}^{(-1)}, K_{-}^{(+1)}\right\rangle\right)=0 .
\end{aligned}
$$

The first two equations of (63) and (64) are gauge covariant and the last ones are local and gauge invariant, as expected.

Let us study first the third equations. Defining the stress-tensor components of $T_{\mu \nu}$ as

$$
T_{++}=\left\langle\Lambda_{+}^{(+1)}, K_{+}^{(-1)}\right\rangle, T_{-+}=-\left\langle\Lambda_{+}^{(+1)}, K_{-}^{(-1)}\right\rangle, T_{+-}=\left\langle\Lambda_{-}^{(-1)}, K_{+}^{(+1)}\right\rangle, T_{--}=-\left\langle\Lambda_{-}^{(-1)}, K_{-}^{(+1)}\right\rangle .
$$

The last equations of (63), (64) become $\partial_{-} T_{++}+\partial_{+} T_{-+}=\partial_{-} T_{+-}+\partial_{+} T_{--}=0$ the conservation laws for the components

$$
\begin{aligned}
& T_{++}=+\frac{1}{2}\left\langle\left(\widehat{A}_{+}^{(L)}\right)^{2}-\left(A_{+}^{(L)}\right)^{2}\right\rangle+\frac{1}{2}\left\langle\psi_{+}^{(+1 / 2)} D_{+}^{(L)} \psi^{(-1 / 2)}\right\rangle+\left\langle\left(Q_{+}^{(0)}\right)^{2}\right\rangle, \\
& T_{--}=+\frac{1}{2}\left\langle\left(\widehat{A}_{-}^{(R)}\right)^{2}-\left(A_{-}^{(R)}\right)^{2}\right\rangle+\frac{1}{2}\left\langle\psi_{-}^{(-1 / 2)} D_{-}^{(R)} \psi^{(+1 / 2)}\right\rangle+\left\langle\left(Q_{-}^{(0)}\right)^{2}\right\rangle, \\
& T_{+-}=T_{-+}=+\left\langle\Lambda_{+}^{(+1)} B \Lambda_{-}^{(-1)} B^{-1}+\frac{1}{2} \psi_{+}^{(+1 / 2)} B \psi_{-}^{(-1 / 2)} B^{-1}\right\rangle .
\end{aligned}
$$

The conserves charge: 13 for these grade \pm 1 equations are

$$
(H+P)=\frac{1}{2} \int_{-\infty}^{+\infty} d x^{1}\left(T_{++}+T_{-+}\right), \quad(H-P)=\frac{1}{2} \int_{-\infty}^{+\infty} d x^{1}\left(T_{--}+T_{+-}\right) .
$$

Now, we analyze the fermionic equations which are the ones we are mainly interested. They are covariant but not gauge invariant and at first sight provide no conservations laws. However, the connections $A_{\mu}^{(L / R)}$ are flat and can be written in the pure gauge form

$$
A_{ \pm}^{(L)}=\partial_{ \pm} g_{L} g_{L}^{-1}, \quad A_{ \pm}^{(R)}=\partial_{ \pm} g_{R} g_{R}^{-1} .
$$

The gauge transformations (34) becomes now

$$
\widetilde{g}_{L}=\Gamma_{L} g_{L} \Gamma_{c L}^{-1}, \quad \widetilde{g}_{R}=\Gamma_{R}^{-1} g_{R} \Gamma_{c R}
$$

where $\Gamma_{c L / R}$ are constant elements of the global part of the gauge group $H_{L} \times H_{R}$.

The flatness of the gauge fields allow to write

$$
D_{ \pm}^{(L)} K_{\mp}^{(-1 / 2)}=g_{L} \partial_{ \pm}\left(g_{L}^{-1} K_{\mp}^{(-1 / 2)} g_{L}\right) g_{L}^{-1}, \quad D_{ \pm}^{(R)} K_{\mp}^{(+1 / 2)}=g_{R} \partial_{ \pm}\left(g_{R}^{-1} K_{\mp}^{(+1 / 2)} g_{R}\right) g_{R}^{-1} .
$$

\footnotetext{
${ }^{13}$ We have used the change of basis

$$
4 T_{00}=T_{++}+T_{--}+2 T_{+-}, 4 T_{01}=T_{++}-T_{--}, T_{10}=T_{01}, T_{11}=T_{++}+T_{--}-2 T_{+-}
$$
}

and set $T_{00}=H$ and $T_{01}=P$. 
Then, we can transform the $\pm 1 / 2$ grade equations into the following non-local fermionic conservation laws

$$
\begin{aligned}
& \partial_{-}\left(g_{L}^{-1} K_{+}^{(-1 / 2)} g_{L}\right)+\partial_{+}\left(-g_{L}^{-1} K_{-}^{(-1 / 2)} g_{L}\right)=0, \\
& \partial_{+}\left(g_{R}^{-1} K_{-}^{(+1 / 2)} g_{R}\right)+\partial_{-}\left(-g_{R}^{-1} K_{+}^{(+1 / 2)} g_{R}\right)=0,
\end{aligned}
$$

because of the presence of the Wilson lines

$$
g_{L / R}(p)=P \exp \left(\int_{p_{0}}^{p} d x^{\mu} A_{\mu}^{(L / R)}\right),
$$

where $p=(t, x) \in \Sigma$ is an arbitrary point and $p_{0} \in \Sigma$ is a fixed reference point 14 .

These equations are gauge invariant under the local part of the gauge group because under (24), we have

$$
\widetilde{K}_{ \pm}^{(-1 / 2)}=\Gamma_{L} K_{ \pm}^{(-1 / 2)} \Gamma_{L}^{-1}, \quad \widetilde{K}_{ \pm}^{(+1 / 2)}=\Gamma_{R}^{-1} K_{ \pm}^{(+1 / 2)} \Gamma_{R}
$$

but transforms under the global part of it by conjugations with $\Gamma_{L / R}^{c}$. However, as we discussed before (section 2.3) the global part of the gauge group preserve the fermionic kernel and it is in this sense that we have well defined conserved charges. They are given by the following $\operatorname{dim} \mathcal{K}_{F}^{( \pm 1 / 2)}$ non-local conserved supercharges $Q\left(\delta_{ \pm 1 / 2}\right)$ associated to the $\delta_{ \pm 1 / 2}$ symmetry flows

$$
\begin{aligned}
& Q\left(\delta_{+1 / 2}\right)=\int_{-\infty}^{+\infty} d x^{1} G\left(\delta_{+1 / 2}\right)=Q_{i}^{+} F_{i}^{(-1 / 2)}, \\
& G\left(\delta_{+1 / 2}\right)=g_{L}^{-1}\left(\left[\psi^{(-1 / 2)}, \widehat{A}_{+}^{(L)}\right]+B \psi_{-}^{(-1 / 2)} B^{-1}\right)^{\perp} g_{L}, \\
& Q\left(\delta_{-1 / 2}\right)=\int_{-\infty}^{+\infty} d x^{1} G\left(\delta_{-1 / 2}\right)=Q_{i}^{-} F_{i}^{(+1 / 2)}, \\
& G\left(\delta_{-1 / 2}\right)=g_{R}^{-1}\left(-\left[\psi^{(+1 / 2)}, \widehat{A}_{-}^{(R)}\right]-B^{-1} \psi_{+}^{(+1 / 2)} B\right)^{\perp} g_{R},
\end{aligned}
$$

where $i=1, \ldots, \operatorname{dim} \mathcal{K}_{F}^{( \pm 1 / 2)}$. The action of $\Gamma_{c L / R}$ on the charges is given by

$$
\widetilde{Q}\left(\delta_{+1 / 2}\right)=\Gamma_{c L}^{-1} Q\left(\delta_{+1 / 2}\right) \Gamma_{c L} \in \mathcal{K}_{F}^{(-1 / 2)}, \quad \widetilde{Q}\left(\delta_{-1 / 2}\right)=\Gamma_{c R} Q\left(\delta_{-1 / 2}\right) \Gamma_{c R}^{-1} \in \mathcal{K}_{F}^{(+1 / 2)}
$$

and, as mentioned above, we expect to obtain an extended global symmetry superalgebra $\mathcal{K} \subset \widehat{\mathfrak{f}} \rightarrow \delta_{\mathcal{K}}$.

From the analysis around (41) we can compute the 2D spin for all the conserved charges extracted from (62). In particular, from (63), (64) we confirm that $G\left(\delta_{ \pm 1 / 2}\right)$ are indeed 2D spinorial currents because the power of the spectral parameter is half-integer, i.e $z^{ \pm 1 / 2}$.

The most interesting situation to be considered and which is related to the Pohlmeyer reductions of superstring sigma models, is when the gauge group $H_{L} \times H_{R}$ is reduced to $H$ and the only gauge field $A_{ \pm}$involved obey the equations of motion provided by (54). In this case the gauge field is also flat but their components are functions of the dynamical fields $B, \psi^{( \pm 1 / 2)}$ turning the conjugations in $Q\left(\delta_{ \pm 1 / 2}\right)$ with the Wilson lines (67) non-trivial. This situation will be addressed elsewhere because we have found some difficulties in trying to obtain the field variations from these non-local charges. Fortunately, in the on-shell gauge $A_{ \pm}^{(L / R)}=0$, the symmetries induced by (68) are symmetries of the field configurations that solve the equations of motion. Below in the examples, we will ignore the Wilson lines and explore a little bit the relation between $Q_{i}^{ \pm}$and some well-known results obtained from superspace in order to motivate further the study of these new $2 \mathrm{D}$ supersymmetries.

\footnotetext{
${ }^{14}$ Under gauge transformations we have $\Gamma_{c L / R}=\Gamma_{L / R}\left(p_{0}\right)$.
} 
Now, in the following we restrict ourselves to the on-shell gauge $A_{ \pm}^{(L / R)}=0$ in which sharper statements can be made. In this gauge we have

$$
\begin{aligned}
& Q\left(\delta_{+1 / 2}\right)=\int_{-\infty}^{+\infty} d x^{1}\left(\left[\psi^{(-1 / 2)}, \partial_{+} B B^{-1}\right]+B \psi_{-}^{(-1 / 2)} B^{-1}\right)^{\perp} \\
& Q\left(\delta_{-1 / 2}\right)=\int_{-\infty}^{+\infty} d x^{1}\left(\left[\psi^{(+1 / 2)}, B^{-1} \partial_{-} B\right]-B^{-1} \psi_{+}^{(+1 / 2)} B\right)^{\perp} .
\end{aligned}
$$

Below, in section 2.7, we shall show by using Poisson brackets, that these supercharges generate the supersymmetry transformations (45), (46) with $A_{ \pm}^{(L / R)}=0$, showing that they are fermionic Hamiltonian flows on the phase space of the system.

When there are no gauge symmetries at all, i.e $\mathcal{K}_{B}^{(0)}=\varnothing$, we recover the supercharges of $[\underline{6}$ for the extended super mKdV hierarchy. The supersymmetry transformations induced by (69) in this case, are exactly of the same form (45), (46) with $A_{ \pm}^{(L / R)}=\theta^{( \pm 1 / 2)}=0$ and are symmetries of the action functional (48). The associated Noether conserved charges are exactly (69) and the same occurs here when $\mathcal{K}_{B}^{(0)} \neq \varnothing$, with a global gauge group, see (98) below. Thus, we conclude that the supercharges extracted from the Drinfeld-Sokolov and Noether procedures coincide.

Finally, we consider the grade zero equations of (63) and (64). They provide the following non-local conserved charges

$$
\Omega_{L / R}=P \exp \left(\frac{1}{2} \int_{-\infty}^{+\infty} d x^{1}\left(A_{+}^{(L / R)}-A_{-}^{(L / R)}\right)\right)
$$

and in the case when the gauge algebras $\mathfrak{h}_{L / R}$ are Abelian, they reduce to

$$
Q_{L / R}=\frac{1}{2} \int_{-\infty}^{+\infty} d x^{1}\left(A_{+}^{(L / R)}-A_{-}^{(L / R)}\right)
$$

Below, in the examples, we will see that these conserved charges encode some symmetry data of the target space of the SSSSG model action (52).

We will end this section by comparing the supersymmetry involved in the supersymmetrization of a WZNW model based on an ordinary Lie algebra with the supersymmetry flows (SF) involved in the formulation of the SSSSG models (52), (55). In the superspace WZNW based on a Lie algebra $\mathfrak{g}$, the bosonic fields are replaced by scalar superfields, as a consequence, bosons $\phi$ and fermions $\psi$ are both parametrized by the same elements in $\mathfrak{g}$ i.e $\phi=\phi^{i} T_{i}, \psi=\psi^{i} T_{i}$, where $T_{i}$ are the generators of $\mathfrak{g}$. In our case the situation is very different, the physical fields parametrize the image part $\mathcal{M}$ of a superalgebra in the decomposition $\widehat{\mathfrak{f}}=\mathcal{K}+\mathcal{M}$, while the symmetries $\delta_{\mathcal{K}}$ are generated by the kernel part $\mathcal{K}$ of it. Due to the fact that $[\mathcal{K}, \mathcal{M}] \subset \mathcal{M}$, we have a map from physical fields to physical fields $\delta_{\mathcal{K}}: \mathcal{M} \rightarrow \mathcal{M}$. Decomposing $\mathcal{M}=\mathcal{M}_{B} \oplus \mathcal{M}_{F}$ in bosonic and fermionic parts and $\mathcal{K}=\mathcal{K}_{B} \oplus \mathcal{K}_{F}$ in a similar way, we see that a supersymmetry flow obeys $\delta_{S F}: \mathcal{M}_{F} \rightarrow \mathcal{M}_{B}$ and $\delta_{S F}: \mathcal{M}_{B} \rightarrow \mathcal{M}_{F}$, mapping the odd part into the even one and viceversa. In a supermatrix representation, such a map is roughly of the form

$$
\begin{array}{ll}
\delta_{S F}: & \left(\begin{array}{cc}
0 & \psi \\
\psi^{\prime} & 0
\end{array}\right) \in \mathcal{M}_{F} \rightarrow\left(\begin{array}{cc}
\delta\left(\psi, \psi^{\prime}\right) & 0 \\
0 & \delta^{\prime}\left(\psi, \psi^{\prime}\right)
\end{array}\right) \in \mathcal{M}_{B} \\
\delta_{S F}: & \left(\begin{array}{cc}
\phi & 0 \\
0 & \phi^{\prime}
\end{array}\right) \in \mathcal{M}_{B} \rightarrow\left(\begin{array}{cc}
0 & \delta\left(\phi, \phi^{\prime}\right) \\
\delta^{\prime}\left(\phi, \phi^{\prime}\right) & 0
\end{array}\right) \in \mathcal{M}_{F} .
\end{array}
$$

Of course we have to guarantee that the number of bosonic and fermionic generators in $\mathcal{M}$ match in the appropriate way for $\delta_{\mathcal{K}}$ to be considered as a supersymmetry. Fortunately, this is guaranteed by the finer $\mathbb{Z}_{4}$ grading decomposition entering the Lie algebraic structure of the integrable hierarchy, which was defined if (4). Recall also that the gauge group $H$ have actions $\delta_{H}$ which mixes with $\delta_{S F}$, i.e $\left[\delta_{H}, \delta_{S F}\right]=\delta_{S F}^{\prime}$. 


\subsection{Bi-Hamiltonian structure of the extended homogeneous hierarchy.}

In this section we introduce two Hamiltonian structures associated to the extended homogeneous hierarchy. They can be extracted directly from the Lax operators but to achieve this it is useful first to introduce the notion of differential of a functional on a co-adjoint orbit $\Xi$. In this section and the next, we assume that the constraints (38) are satisfied 15 .

Start by introducing the integrated inner product

$$
(X, Y)=\int_{-\infty}^{+\infty} d x^{1}\langle X, Y\rangle,
$$

with $\langle X, Y\rangle_{\widehat{f}}$ is defined as in (15). The strategy is to look for the two Hamiltonian structures for functionals defined on the co-adjoint orbits $L_{+} \in \widehat{\mathfrak{f}}^{*}$ and $L_{-}^{\prime} \in \widehat{\mathfrak{f}}^{*}$. From these "light-cone" orbits we select the true phase space as the spatial component of the Lax operator, i.e $L_{x}=\frac{1}{2}\left(L_{+}-L_{-}\right)$. Note that the relativistic counterpart of $L_{+}$is taken as $L_{-}^{\prime}$ and not $L_{-}$, which is more natural as can be seen from the equations (32), (33). Now that $L_{+}, L_{-}^{\prime}$ are considered as phase spaces, denoted generically by $\Xi$, it is useful to recall the definition of a differential $d \Xi h$ of a functional on the orbit $\Xi$, i.e of $h(\Xi): \Xi \rightarrow \mathcal{F}\left(\mathcal{F}\right.$ is a field), which is a linear form in $\left(\widehat{\mathfrak{f}}^{*}\right)^{*} \sim \widehat{\mathfrak{f}}$. Then, with $\Xi \in \widehat{\mathfrak{f}}^{*}$ and a function $h$, we find the differential $d \Xi h$ of $h$ through the taylor-like relation

$$
h(\Xi+\delta \Xi)=h(\Xi)+\left(d_{\Xi} h\right) \circ(\delta \Xi)+\mathcal{O}\left(\delta \Xi^{2}\right),
$$

where $\delta \Xi \in \widehat{\mathfrak{f}}^{*}$ is an arbitrary variation of $\Xi$.

Under the inner products (171) or (15), $\widehat{\mathfrak{f}}$ and its dual $\widehat{\mathfrak{f}}^{*}$ are identified and we can write $\left(d_{\Xi} h\right) \circ(\delta \Xi)=(d \Xi h, \delta \Xi)$. When the orbit $\Xi$ takes values on some subspace $\widehat{\mathfrak{f}} \Xi \subset \widehat{\mathfrak{f}}$ of the Lie algebra $\widehat{\mathfrak{f}}$, we write the variation in the form $\delta \Xi=\varepsilon r_{\Xi}$, where $\varepsilon<<1, r_{\Xi} \in \widehat{\mathfrak{f}}_{\Xi}$ and this leads to the following definition of the differential of a function 16 on $\Xi$

$$
\left.\frac{d}{d \varepsilon} h\left(\Xi+\varepsilon r_{\Xi}\right)\right|_{\varepsilon=0} \equiv\left(d_{\Xi} h, r_{\Xi}\right)
$$

where we need to compute explicitly the LHS in order to isolate the differential $d_{\Xi} h$ in the RHS. From this we immediately conclude that the differentia $17 d_{\Xi} \in \widehat{\widehat{f}} \doteq$ belongs to the ortho-complement of $\widehat{\mathfrak{f}} \Xi$ in $\widehat{\mathfrak{f}}$ because of the operator $\mathcal{P}_{\perp}(*)=\left(r_{\Xi}, *\right): \widehat{\mathfrak{f}} \Xi \rightarrow \widehat{\mathfrak{f}}_{\Xi}^{\perp}$ is a projector along $\widehat{\mathfrak{f}}_{\Xi}^{\perp}$.

Under an arbitrary conjugation $\widetilde{\Xi}=S \Xi S^{-1}$, we get $r_{\Xi}=S r_{\Xi} S^{-1}$ and from (72) we can see the effect of a conjugation on a differential, which is given by $d_{\Xi} h=S\left(d_{\Xi} h\right) S^{-1}$. This relation has to be used in order to find the differentials on $L_{+}^{\prime}, L_{-}$starting from those differentials computed on the orbits $L_{+}, L_{-}^{\prime}$, recall that we have $L_{ \pm}^{\prime}=B^{-1} L_{ \pm} B$, c.f (19). We denote the differentials of a functional $h$ defined on $L_{+}$and $L_{-}^{\prime}$ as $d_{+} h$ and $d_{-}^{\prime} h$ respectively, and a similar notation for $L_{+}^{\prime}$ and $L_{-}$after the $B$-conjugation.

Now, we proceed to compute the differentials of the functions we are most interested, namely the conserved charges found in section 2.5 by means of the Drinfeld-Sokolov procedure.

Consider the stress-tensor components $T_{\mu \nu}$ extracted from the DS procedure as functionals on $L_{ \pm}, L_{ \pm}^{\prime}$. They are

$T_{++}=\left\langle\Lambda_{+}^{(+1)}, U_{-}^{-1} L_{+} U_{-}\right\rangle, T_{-+}=-\left\langle\Lambda_{+}^{(+1)}, U_{-}^{-1} L_{-} U_{-}\right\rangle, T_{+-}=\left\langle\Lambda_{-}^{(-1)}, U_{+}^{-1} L_{+}^{\prime} U_{+}\right\rangle, T_{--}=-\left\langle\Lambda_{-}^{(-1)}, U_{+}^{-1} L_{-}^{\prime} U_{+}\right\rangle$,

where we have used (57) and (59) in the $A=0$ gauge. Taking into account the variation 18 $\delta L_{+}=\varepsilon r_{\geq 0}, \delta L_{-}^{\prime}=\varepsilon r_{\leq 0}$, the fact that $U_{ \pm}(\varepsilon)$ depend on $\varepsilon$ through the fields, the ad-invariance of the inner product and the relation $\left[\Lambda_{ \pm}^{( \pm 1)}, L_{ \pm}^{V}\right]=0$,

\footnotetext{
${ }^{15}$ In particular, this happens for the soliton solutions constructed from the dressing method, e.g see [9].

${ }^{16}$ This definition is equivalent to the usual notion of functional differentiation after taking the trace [15].

${ }^{17}$ The use of the symbol $\perp$ should not be confuse with the same symbol used before to denote projection along the kernel algebra $\mathcal{K}$.

${ }^{18}$ The $r_{\geq 0}, r_{\leq 0}$ stands for arbitrary terms taking values on the domains of the Lax connections $\Lambda_{\Theta}^{(+1)}, \Lambda_{\Pi^{\prime}}^{(-1)}$.
} 
where $L_{ \pm}^{V}$ are the vacuum Lax operators, we get from (72), the associated differentials

$$
\begin{array}{ll}
d_{+} T_{++}=\left(\Theta \Lambda_{+}^{(+1)} \Theta^{-1}\right)_{\leq 0}, & d_{-}^{\prime} T_{-+}=-\left(\Theta^{\prime} \Lambda_{+}^{(+1)} \Theta^{\prime-1}\right)_{\geq 0}, \\
d_{+} T_{+-}=\left(\Pi \Lambda_{-}^{(-1)} \Pi^{-1}\right)_{\leq 0}, & d_{-}^{\prime} T_{--}=-\left(\Pi^{\prime} \Lambda_{-}^{(-1)} \Pi^{\prime-1}\right)_{\geq 0},
\end{array}
$$

where we have used the factorizations $\Theta=U_{-} S_{-}, \Pi=B U_{+} S_{+}, \Theta^{\prime}=B^{-1} \Theta, \Pi^{\prime}=U_{+} S_{+}$and the fact that the kernel components $S_{ \pm}$does not contribute.

In a very similar way but this time using the fact that $\widehat{\mathfrak{f}}^{\perp} \cap \widehat{\mathfrak{f}}^{\|}=\oslash$, we have for the fermionic functions

$$
\begin{array}{ll}
m_{+}=\left(D^{(+1 / 2)}, K_{+}^{(-1 / 2)}\right), & m_{-}=-\left(D^{(+1 / 2)}, K_{-}^{(-1 / 2)}\right), \\
n_{+}=-\left(D^{(-1 / 2)}, K_{+}^{(+1 / 2)}\right), & n_{-}=\left(D^{(-1 / 2)}, K_{-}^{(+1 / 2)}\right),
\end{array}
$$

where $D^{( \pm 1 / 2)}=\epsilon_{i} F_{i}^{( \pm 1 / 2)}, i=1, \ldots, \operatorname{dim} \mathcal{K}_{F}^{( \pm 1 / 2)}$, the associated differentials

$$
\begin{array}{ll}
d_{+} m_{+}=\left(U_{-} D^{(+1 / 2)} U_{-}^{-1}\right)_{\leq 0}, & d_{-}^{\prime} m_{-}=-B^{-1}\left(U_{-} D^{(+1 / 2)} U_{-}^{-1}\right)_{\geq 0} B, \\
d_{+} n_{+}=-B\left(U_{+} D^{(-1 / 2)} U_{+}^{-1}\right)_{\leq 0} B^{-1}, & d_{-}^{\prime} n_{-}=\left(U_{+} D^{(-1 / 2)} U_{+}^{-1}\right)_{\geq 0} .
\end{array}
$$

To identify the Poisson structures, we follow the approach adopted in [15] to study the generalized Drinfeld-Sokolov hierarchies and apply those results to our extended homogeneous hierarchy. For ease of simplicity, we perform the calculation for a bosonic hierarchy with an integer homogeneous gradation and at the end we comment on the necessary changes required in the supersymmetric case.

Consider $L_{+}$as the phase space and write the equations of motion $\left[L_{+}, L_{-}\right]=0$ with $L_{-}=\partial_{-}-\left(\Pi \Lambda_{-}^{(-1)} \Pi^{-1}\right)_{<0}$, in the following two equivalent Lax forms

$$
\partial_{-} L_{+}=\left[\left(\Pi \Lambda_{-}^{(-1)} \Pi^{-1}\right)_{<0}, L_{+}\right]=-\left[\left(\Pi \Lambda_{-}^{(-1)} \Pi^{-1}\right)_{\geq 0}, L_{+}\right] .
$$

The first and second terms on the RHS of (75) will lead, respectively, to the first and second Poisson structures defined on $L_{+}$as we now see.

Considering the first form of $\partial_{-} L_{+}$and using $z^{-1}\left(\Pi z \Lambda_{-}^{(-1)} \Pi^{-1}\right)_{\leq 0}=\left(\Pi \Lambda_{-}^{(-1)} \Pi^{-1}\right)_{<0}$, because $z$ have homogeneous grade +1 , we find that

$$
z^{-1} d_{+} T_{+-}(z)=\left(\Pi \Lambda_{-}^{(-1)} \Pi^{-1}\right)_{<0}
$$

where $d_{+} T_{+-}(z)$ means that $\Lambda_{-}^{(-1)}$ is replaced by $z \Lambda_{-}^{(-1)}$ in the definition of $d_{+} T_{+_{-}}$. Then, we have the differential representation of the first form

$$
\partial_{-} L_{+}=\left[z^{-1} d_{+} T_{+-}(z), L_{+}\right]_{\geq 0},
$$

where we have, for consistency, projected along the same grade space decomposition of the Lax connection in $L_{+}$.

Now, for the second form of $\partial_{-} L_{+}$we use $z\left(\Pi z^{-1} \Lambda_{-}^{(-1)} \Pi^{-1}\right)_{\geq 0}=\left(\Pi \Lambda_{-}^{(-1)} \Pi^{-1}\right)_{>0}$, because $z^{-1}$ have homogeneous grade -1 , giving

$$
\partial_{-} L_{+}=-\left[\left(d_{+} T_{+-}\right)_{0}, L_{+}\right]-z\left[\left(\Pi z^{-1} \Lambda_{-}^{(-1)} \Pi^{-1}\right)_{\geq 0}, L_{+}\right] .
$$

Noting that $\partial_{z^{-1}(-)} L_{+}=-\left[\left(\Pi z^{-1} \Lambda_{-}^{(-1)} \Pi^{-1}\right)_{\geq 0}, L_{+}\right]$, where $\partial_{z^{-1}(-)} L_{+}$means that $\Lambda_{-}^{(-1)}$ is replaced by $z^{-1} \Lambda_{-}^{(-1)}$, we use the first form representation (76) to write $\partial_{z^{-1}(-)} L_{+}=\left[z^{-1} d_{+} T_{+-}, L_{+}\right]_{\geq 0}$ and obtain

$$
\partial_{-} L_{+}=-\left[\left(d_{+} T_{+-}\right)_{0}, L_{+}\right]+z\left[z^{-1} d_{+} T_{+-}, L_{+}\right]_{\geq 0} \text {. }
$$


Finally, using $z\left[z^{-1} d_{+} T_{+-}, L_{+}\right]_{\geq 0}=\left[d_{+} T_{+-}, L_{+}\right]_{>0}$, we obtain the differential representation of the second form

$$
\partial_{-} L_{+}=-\left[\left(d_{+} T_{+-}\right)_{0}, L_{+}\right]+\left[d_{+} T_{+-}, L_{+}\right]_{>0} .
$$

From these results we can write two equivalent forms for the $\partial_{-}$evolution of a functional $\varphi$ on $L_{+}$, i.e

$$
\begin{aligned}
& \partial_{-}=\left(d_{+} \varphi, \partial_{-} L_{+}\right)=\left(d_{+} \varphi,\left[z^{-1} d_{+} T_{+-}(z), L_{+}\right]\right), \\
& \partial_{-}=\left(d_{+} \varphi, \partial_{-} L_{+}\right)=\left(d_{+} \varphi,-\left[\left(d_{+} T_{+-}\right)_{0}, L_{+}\right]+\left[d_{+} T_{+-}, L_{+}\right]_{>0}\right),
\end{aligned}
$$

leading to the following two Kostant-Kirillov brackets on the orbit $L_{+}$

$$
\begin{aligned}
& \{\varphi, \psi\}_{1}\left(L_{+}\right)=-\left(L_{+}, z^{-1}\left[d_{+} \varphi, d_{+} \psi\right]\right), \\
& \{\varphi, \psi\}_{2}\left(L_{+}\right)=\left(L_{+},\left[\left(d_{+} \varphi\right)_{0},\left(d_{+} \psi\right)_{0}\right]-\left[\left(d_{+} \varphi\right)_{<0},\left(d_{+} \psi\right)_{<0}\right]\right),
\end{aligned}
$$

where we have used the decomposition $d_{+} f=\left(d_{+} f\right)_{0}+\left(d_{+} f\right)_{<0}$ in order to simplify the second bracket. From (79) we can write (178) as a recursion relation

$$
\partial_{-} \varphi=\left\{T_{+-}(z), \varphi\right\}_{1}\left(L_{+}\right)=\left\{T_{+-}, \varphi\right\}_{2}\left(L_{+}\right) .
$$

In a complete analogous way, we consider $L_{-}^{\prime}$ as the phase space and write $\left[L_{+}^{\prime}, L_{-}^{\prime}\right]=0$ in the two equivalent Lax forms. We get

$$
\begin{aligned}
\partial_{+} L_{-}^{\prime} & =\left[z d_{-}^{\prime} T_{-+}\left(z^{-1}\right), L_{-}^{\prime}\right]_{\leq 0}, \\
\partial_{+} L_{-}^{\prime} & =-\left[\left(d_{-}^{\prime} T_{-+}\right)_{0}, L_{-}^{\prime}\right]+\left[d_{-}^{\prime} T_{-+}, L_{-}^{\prime}\right]_{<0},
\end{aligned}
$$

leading to the following two Kostant-Kirillov brackets on the orbit $L_{-}^{\prime}$

$$
\begin{aligned}
& \{\varphi, \psi\}_{1}\left(L_{-}^{\prime}\right)=\left(L_{-}^{\prime}, z\left[d_{-}^{\prime} \varphi, d_{-}^{\prime} \psi\right]\right) \\
& \{\varphi, \psi\}_{2}\left(L_{-}^{\prime}\right)=-\left(L_{-}^{\prime},\left[\left(d_{-}^{\prime} \varphi\right)_{0},\left(d_{-}^{\prime} \psi\right)_{0}\right]-\left[\left(d_{-}^{\prime} \varphi\right)_{>0},\left(d_{-}^{\prime} \psi\right)_{>0}\right]\right),
\end{aligned}
$$

where we have, for convenience, multiplied by a global factor -1 . The recursion relation becomes now

$$
\partial_{+} \varphi=-\left\{T_{-+}\left(z^{-1}\right), \varphi\right\}_{1}\left(L_{-}^{\prime}\right)=-\left\{T_{-+}, \varphi\right\}_{2}\left(L_{-}^{\prime}\right) .
$$

It is instructive to repeat the same analysis for the identities $\left[L_{+}, L_{+}\right]=0$ and $\left[L_{-}^{\prime}, L_{-}^{\prime}\right]=0$. We find that $\partial_{+} L_{+}$ and $\partial_{-} L_{-}^{\prime}$ can be written in two equivalent ways

$$
\begin{aligned}
& \partial_{+} L_{+}=\left[z^{-1} d_{+} T_{++}(z), L_{+}\right]_{\geq 0}=-\left[\left(d_{+} T_{++}\right)_{0}, L_{+}\right]+\left[d_{+} T_{++}, L_{+}\right]_{>0}, \\
& \partial_{-} L_{-}^{\prime}=\left[z d_{-}^{\prime} T_{--}\left(z^{-1}\right), L_{-}^{\prime}\right]=-\left[\left(d_{-}^{\prime} T_{--}\right)_{0}, L_{-}^{\prime}\right]+\left[d_{-}^{\prime} T_{--}, L_{-}^{\prime}\right]_{<0},
\end{aligned}
$$

showing that the mixed components of the stress-tensor, i.e $T_{ \pm \mp}$, are responsible for coupling the two sectors of the relativistic part of the extended integrable hierarchy as shown by (76), (77).

The brackets (79), (82) can be written in the following compact $r$-bracket forms

$$
\begin{aligned}
& \{\varphi, \psi\}_{\mu}\left(L_{+}\right)=\left(L_{+},\left[d_{+} \varphi, d_{+} \psi\right]_{\mathcal{R}_{<, \mu}}\right), \\
& \{\varphi, \psi\}_{\nu}\left(L_{-}^{\prime}\right)=\left(L_{-}^{\prime},\left[d_{-}^{\prime} \varphi, d_{-}^{\prime} \psi\right]_{\mathcal{R}_{>, \nu}}\right),
\end{aligned}
$$


where $(\mu, \nu \in \mathbb{C})$

$$
\begin{array}{lll}
\mathcal{R}_{<, \mu}=\left(\mathcal{R}_{<}-\mu \cdot z^{-1}\right), & \mathcal{R}_{<}=\frac{1}{2}\left(\mathcal{P}_{0}-\mathcal{P}_{<0}\right), \\
\mathcal{R}_{>, \nu}=-\left(\mathcal{R}_{>}-\nu \cdot z\right), & \mathcal{R}_{>}=\frac{1}{2}\left(\mathcal{P}_{0}-\mathcal{P}_{>0}\right),
\end{array}
$$

are the corresponding $r$-matrices entering the $r$-bracket $19[X, Y]_{\mathcal{R}}=[\mathcal{R}(X), Y]+[X, \mathcal{R}(Y)]$ and $\mathcal{P}_{0}, \mathcal{P}_{<0}, \mathcal{P}_{>0}$ are the projectors along zero, negative and positive grades.

The Jacobi identity and the compatibility of (84) are guaranteed because each $r$-matrix $\mathcal{R}_{<, \mu}, \mathcal{R}_{>, \nu}$ satisfy separately the classical modified Yang-Baxter equation

$$
\left[\mathcal{R}_{i}(X), \mathcal{R}_{i}(Y)\right]-\mathcal{R}_{i}\left(\left[\mathcal{R}_{i}(X), Y\right]+\left[X, \mathcal{R}_{i}(Y)\right]\right)=\lambda_{i}[X, Y], \quad \lambda_{i} \in \mathbb{C}, i=1,2
$$

with $\mathcal{R}_{1}=\mathcal{R}_{<, \mu}, \mathcal{R}_{2}=\mathcal{R}_{>, \nu}$ and

$$
\lambda_{1}=-\left(\frac{1}{2}+\mu \cdot z^{-1}\right)^{2}, \quad \lambda_{2}=-\left(\frac{1}{2}+\nu \cdot z\right)^{2} .
$$

In what follows we will choose the second symplectic structure because, for the homogeneous hierarchies, it is nothing but the canonical symplectic structure associated to the Lagrangian (48), see (101) below. In the superalgebra case, we only have to replace trace by supertrace and the integer gradation by half-integer one. For the moment, it is of great importance to mention that the form of the first Hamiltonian structure above is a consequence of the special properties of the only gradation (naively) used, i.e, the integer homogenous gradation. Note that we are not taking into account any other gradations. This does not necessarily mean that the integrable system described by the action (48) admits such an structure in the form presented above, i.e, that the supersymmetrization of the first structure takes the same form as in (79), (82), because the existence of the finer $\mathbb{Z}_{4}$ decomposition entering in (3) could preclude it. In that case, the recursion relations (80), (83) have to be modified in an appropriate way and this changes the explicit form of the first Poisson structure, in fact it becomes non-local [10. However, the second structure is enough for our present purpose 20 .

The $r$-matrices (85) satisfy (86) separately and based on this fact we now propose a bracket on $L_{x}$ through the following definition.

Definition 2 The bracket on the spatial orbit $L=L_{x}$ is given by

$$
\{\varphi, \psi\}_{2}(L)=\{\varphi, \psi\}_{2}\left(L_{+}\right)-\{\varphi, \psi\}_{2}\left(L_{-}\right)=\left(L_{+},\left[d_{+} \varphi, d_{+} \psi\right]_{\mathcal{R}_{<}}\right)+\left(L_{-},\left[d_{-} \varphi, d_{-} \psi\right]_{\mathcal{R}_{>}}\right),
$$

where we find $\{\varphi, \psi\}_{2}\left(L_{-}\right)$and the differentials $d_{-} \varphi, d_{-} \psi$ by using the map $L_{-}^{\prime}=B^{-1} L_{-} B$. The differentials $d \varphi, d \psi$ on $L$ are constructed by restricting the functionals $\varphi, \psi$ on the respective domains of definitions of $L_{+}$and $L_{-}^{\prime}$. The bracket for the B-equivalent representation $L^{\prime}$ is defined accordingly.

For computational purposes, we use $L_{+}=\partial_{+}+\Lambda_{\Theta}^{(+1)}$ and $L_{-}^{\prime}=\partial_{-}-\Lambda_{\Pi^{\prime}}^{(-1)}$ in order to rewrite the brackets expressions. We get 22

$$
\begin{aligned}
& \{\varphi, \psi\}_{2}\left(L_{+}\right)=\left(\Lambda_{\Theta}^{(+1)},\left[d_{+} \varphi, d_{+} \psi\right]_{\mathcal{R}_{<}}\right)+\left(\partial_{+}\left(d_{+} \varphi\right), \mathcal{R}_{<}\left(d_{+} \psi\right)\right)-\left(\mathcal{R}_{<}\left(d_{+} \varphi\right), \partial_{+}\left(d_{+} \psi\right)\right), \\
& \{\varphi, \psi\}_{2}\left(L_{-}^{\prime}\right)=\left(\Lambda_{\Pi^{\prime}}^{(-1)},\left[d_{-}^{\prime} \varphi, d_{-}^{\prime} \psi\right]_{\mathcal{R}_{>}}\right)-\left(\partial_{-}\left(d_{-}^{\prime} \varphi\right), \mathcal{R}_{>}\left(d_{-}^{\prime} \psi\right)\right)+\left(\mathcal{R}_{>}\left(d_{-}^{\prime} \varphi\right), \partial_{-}\left(d_{-}^{\prime} \psi\right)\right),
\end{aligned}
$$

\footnotetext{
${ }^{19}$ Explicitly: $[X, Y]_{\mathcal{R}_{\lessgtr}}= \pm\left(\left[X_{0}, Y_{0}\right]-\left[X_{\lessgtr^{0}}, Y_{\lessgtr}\right]\right)$.

${ }^{20}$ The first bracket is requiered only when we want to link the sigma/SSSSG model symplectic structures.

${ }^{21}$ We have absorbed the $1 / 2$ in $L=\frac{1}{2}\left(L_{+}-L_{-}\right)$.

${ }^{22}$ Concerning the first expression right below, a similar bracket was introduce in [16] by using a superspace approach to the Drinfeld-Sokolov reduction.
} 
where we have used the ad-invariance of the inner product to write

$$
\left(\partial,[X, Y]_{\mathcal{R}}\right)=(\partial X, \mathcal{R}(Y))-(\mathcal{R}(X), \partial Y) .
$$

Note that these derivative terms only receive contributions from the zero grade parts of the algebra.

\subsection{The second Hamiltonian structure: the supercharges algebra and the field varia- tions.}

The purpose of this section is threefold. We will find part of the algebra obeyed by the supercharges (69) and comment later on some issues related to the role of gauge group. We shall obtain, by using the second Poisson bracket (87), the supersymmetric field variations induced by the supercharges and also show that the second symplectic structure found above coincides with the symplectic structure of the WZNW model (48).

Let us start with the following proposition.

Proposition 3 Under the second Poisson structure, the functionals 73) satisfy the relations

$$
\begin{aligned}
\left\{m_{+}, \widetilde{m}_{+}\right\}_{2}\left(L_{+}\right) & =2 \epsilon \cdot \widetilde{\epsilon} \int_{-\infty}^{+\infty} d x^{1} T_{++}, & \left\{m_{-}, \widetilde{m}_{-}\right\}_{2}\left(L_{-}\right) & =-2 \epsilon \cdot \widetilde{\epsilon} \int_{-\infty}^{+\infty} d x^{1} T_{-+} \\
\left\{n_{+}, \widetilde{n}_{+}\right\}_{2}\left(L_{+}^{\prime}\right) & =2 \bar{\epsilon} \cdot \widetilde{\bar{\epsilon}} \int_{-\infty}^{+\infty} d x^{1} T_{+-}, & \left\{n_{-}, \widetilde{n}_{-}\right\}_{2}\left(L_{-}^{\prime}\right) & =-2 \bar{\epsilon} \cdot \widetilde{\bar{\epsilon}} \int_{-\infty}^{+\infty} d x^{1} T_{--} .
\end{aligned}
$$

Moreover, under (87) the supercharges (69) written as $Q^{+}=m_{+}+m_{-}, Q^{-}=n_{+}+n_{-}$satisfy

$$
\left\{Q^{+}, \widetilde{Q}^{+}\right\}_{2}(L)=2 \epsilon \cdot \widetilde{\epsilon}(H+P), \quad\left\{Q^{-}, \widetilde{Q}^{-}\right\}_{2}\left(L^{\prime}\right)=2 \bar{\epsilon} \cdot \widetilde{\bar{\epsilon}}(H-P) .
$$

Proof. We will compute one of the brackets only, as the others computations are quite similar. Starting with, cf. (888),

$$
\left\{m_{+}, \widetilde{m}_{+}\right\}_{2}\left(L_{+}\right)=\left(\Lambda_{\Theta}^{(+1)},\left[d_{+} m_{+}, d_{+} \widetilde{m}_{+}\right]_{\mathcal{R}_{<}}\right)+\left(\partial_{+}\left(d_{+} m_{+}\right), \mathcal{R}_{<}\left(d_{+} \widetilde{m}_{+}\right)\right)-\left(\mathcal{R}_{<}\left(d_{+} m_{+}\right), \partial_{+}\left(d_{+} \widetilde{m}_{+}\right)\right),
$$

using the explicit form of the Lax connection

$$
\Lambda_{\Theta}^{(+1)}=A_{+}^{(0)}+Q_{+}^{(0)}+\psi_{+}^{(+1 / 2)}+\Lambda_{+}^{(+1)},
$$

the involved $r$-matrix $\mathcal{R}_{<}=\frac{1}{2}\left(\mathcal{P}_{0}-\mathcal{P}_{<0}\right)$ and the projected components of the differentials

$$
\begin{aligned}
\left(d_{+} m_{+}\right)_{0} & =\left[\psi^{(-1 / 2)}, D^{(+1 / 2)}\right] \\
\left(d_{+} m_{+}\right)_{-1 / 2} & =\left[\psi^{(-1)}, D^{(+1 / 2)}\right]+\frac{1}{2}\left[\psi^{(-1 / 2)},\left[\psi^{(-1 / 2)}, D^{(+1 / 2)}\right]\right],
\end{aligned}
$$

we identify the relevant contributions we need to compute:

$$
\begin{aligned}
& A=\left(\Lambda_{\Theta}^{(+1)},\left[\left(d_{+} m_{+}\right)_{0},\left(d_{+} \widetilde{m}_{+}\right)_{0}\right]\right)=\left(Q_{+}^{(0)},\left[\left[\psi^{(-1 / 2)}, D^{(+1 / 2)}\right],\left[\psi^{(-1 / 2)}, \widetilde{D}^{(+1 / 2)}\right]\right]\right), \\
& B=\left(\Lambda_{\Theta}^{(+1)},\left[\left(d_{+} m_{+}\right)_{-1 / 2},\left(d_{+} \widetilde{m}_{+}\right)_{-1 / 2}\right]\right)=\left(\Lambda_{+}^{(+1)},\left[\left[\psi^{(-1)}, D^{(+1 / 2)}\right],\left[\psi^{(-1)}, \widetilde{D}^{(+1 / 2)}\right]\right]\right), \\
& C=\text { derivative term }=\frac{1}{2}\left(\partial_{+} \psi^{(-1 / 2)},\left[D^{(+1 / 2)},\left[\psi^{(-1 / 2)}, \widetilde{D}^{(+1 / 2)}\right]\right]-\left[\widetilde{D}^{(+1 / 2)},\left[\psi^{(-1 / 2)}, D^{(+1 / 2)}\right]\right]\right) .
\end{aligned}
$$

Considering $A$, we anti-symmetrize and use the ad-invariance of the inner product to get

$$
A=\frac{1}{2}\left(\left[Q_{+}^{(0)},\left[\psi^{(-1 / 2)}, D^{(+1 / 2)}\right]\right],\left[\psi^{(-1 / 2)}, \widetilde{D}^{(+1 / 2)}\right]\right)-\frac{1}{2}\left(\left[Q_{+}^{(0)},\left[\psi^{(-1 / 2)}, \widetilde{D}^{(+1 / 2)}\right]\right],\left[\psi^{(-1 / 2)}, D^{(+1 / 2)}\right]\right) .
$$


Now, use the Jacobi identity to write

$$
\left[Q_{+}^{(0)},\left[\psi^{(-1 / 2)}, X\right]\right]=-\left[X,\left[Q_{+}^{(0)}, \psi^{(-1 / 2)}\right]\right]-\left[\psi^{(-1 / 2)},\left[X, Q_{+}^{(0)}\right]\right],
$$

where $X=D^{(+1 / 2)}$ and $\widetilde{D}^{(+1 / 2)}$. Using the ad-invariance of the inner product again we obtain

$$
\begin{aligned}
A= & \left(\left[Q_{+}^{(0)}, \psi^{(-1 / 2)}\right],\left[D^{(+1 / 2)},\left[\psi^{(-1 / 2)}, \widetilde{D}^{(+1 / 2)}\right]\right]-\left[\widetilde{D}^{(+1 / 2)},\left[\psi^{(-1 / 2)}, D^{(+1 / 2)}\right]\right]\right)- \\
& -\frac{1}{2}\left(\left[Q_{+}^{(0)}, D^{(+1 / 2)}\right],\left[\psi^{(-1 / 2)},\left[\psi^{(-1 / 2)}, \widetilde{D}^{(+1 / 2)}\right]\right]\right)+\frac{1}{2}\left(\left[Q_{+}^{(0)}, \widetilde{D}^{(+1 / 2)}\right],\left[\psi^{(-1 / 2)},\left[\psi^{(-1 / 2)}, D^{(+1 / 2)}\right]\right]\right) .
\end{aligned}
$$

The second line right above vanishes because of the anti-commutativity of the constant Grassmannian parameters $\epsilon, \widetilde{\epsilon}$ and the supersymmetry of the trace and we get

$$
A=\left(\left[Q_{+}^{(0)}, \psi^{(-1 / 2)}\right],\left[D^{(+1 / 2)},\left[\psi^{(-1 / 2)}, \widetilde{D}^{(+1 / 2)}\right]\right]-\left[\widetilde{D}^{(+1 / 2)},\left[\psi^{(-1 / 2)}, D^{(+1 / 2)}\right]\right]\right) .
$$

Using once again the Jacobi identity we obtain

$$
\left[D^{(+1 / 2)},\left[\psi^{(-1 / 2)}, \widetilde{D}^{(+1 / 2)}\right]\right]-\left[\widetilde{D}^{(+1 / 2)},\left[\psi^{(-1 / 2)}, D^{(+1 / 2)}\right]\right]=\left[\psi^{(-1 / 2)},\left[D^{(+1 / 2)}, \widetilde{D}^{(+1 / 2)}\right]\right]=2 \epsilon \cdot \widetilde{\epsilon}\left[\psi^{(-1 / 2)}, \Lambda_{+}^{(+1)}\right]
$$

where $\epsilon \cdot \tilde{\epsilon}=\epsilon_{i} \tilde{\epsilon}_{i}, i=1, \ldots, \operatorname{dim} \mathcal{K}_{F}^{(+1 / 2)}$ and where we have assumed that 23

$$
\left\{F_{i}^{(+1 / 2)}, F_{j}^{(+1 / 2)}\right\}=2 \delta_{i j} \Lambda_{+}^{(+1)}, \quad i=1, \ldots, \operatorname{dim} \mathcal{K}_{F}^{(+1 / 2)} .
$$

Now, we use the ad-invariance of the inner product and the relations (26) to obtain

$$
A=2 \epsilon \cdot \widetilde{\epsilon}\left(\left(Q_{+}^{(0)}\right)^{2}\right), C=2 \epsilon \cdot \widetilde{\epsilon} \frac{1}{2}\left(\psi_{+}^{(+1 / 2)} \partial_{+} \psi^{(-1 / 2)}\right)
$$

where we have used (91) to find $C$.

It remains to compute $B$. Anti-symmetrizing and using ad-invariance of the trace we get

$$
B=\frac{1}{2}\left(\left[\Lambda_{+}^{(+1)},\left[\psi^{(-1)}, D^{(+1 / 2)}\right]\right],\left[\psi^{(-1)}, \widetilde{D}^{(+1 / 2)}\right]\right)-\frac{1}{2}\left(\left[\Lambda_{+}^{(+1)},\left[\psi^{(-1)}, \widetilde{D}^{(+1 / 2)}\right]\right],\left[\psi^{(-1)}, D^{(+1 / 2)}\right]\right) .
$$

Using the Jacobi identity, the fact that $\left[\Lambda_{+}^{(+1)}, X\right]=0$, with $X$ as above and the relations (26) we write

$$
\left[\Lambda_{+}^{(+1)},\left[\psi^{(-1)}, X\right]\right]=\left[X, A_{-}^{(0)}\right] .
$$

This last result, the equation (91) with $\psi^{(-1 / 2)} \rightarrow \psi^{(-1)}$ and once again (26) gives

$$
B=-2 \epsilon \cdot \widetilde{\epsilon} \frac{1}{2}\left(\left(A_{+}^{(0)}\right)^{2}\right) .
$$

Finally, putting all together, i.e $A-B+C$ we arrive to the final expression

$$
\left\{m_{+}, \widetilde{m}_{+}\right\}_{2}\left(L_{+}\right)=2 \epsilon \cdot \tilde{\epsilon} \int_{-\infty}^{+\infty} d x^{1} T_{++}
$$

where we have used (31) in the gauge $A=0$. A similar computation follows for the other brackets, the only difference is that for the brackets involving the mixed components $T_{-+}, T_{+-}$we have to use the fermion equations of motion in (37) and also the relation $\left\{F_{i}^{(-1 / 2)}, F_{j}^{(-1 / 2)}\right\}=-2 \delta_{i j} \Lambda_{-}^{(-1)}$. The supercharge algebra follows directly after noting that

\footnotetext{
${ }^{23}$ In particular, this is satisfied by the superalgebras entering the Pohlmeyer reduction of $A d S_{n} \times S^{n}, n=2,3,5$ and $A d S_{4} \times \mathbb{C} P^{3}$ superstring sigma models. For explicit examples, see below (147) and (153).
} 
the differentials defined on $L_{+}$and $L_{-}^{\prime}$ vanishes when restricted to $L_{-}^{\prime}$ and $L_{+}$respectively, getting $\left\{Q^{+}, \widetilde{Q}^{+}\right\}_{2}(L)=$ $\left\{m_{+}, \widetilde{m}_{+}\right\}_{2}\left(L_{+}\right)-\left\{m_{-}, \widetilde{m}_{-}\right\}_{2}\left(L_{-}\right)$and a similar relation for $\left\{Q^{-}, \widetilde{Q}^{-}\right\}_{2}\left(L^{\prime}\right)$. Taking into account (66), the result follows.

Now, in order to find the supersymmetry field variations we need to find the differentials associated to the physical fields, which are the simplest to find. Let us first introduce the following quantities

$$
\begin{aligned}
G_{\mu}^{(-1 / 2)} & =\mu_{i} G_{i}^{(-1 / 2)}, G_{\nu}^{(+1 / 2)}=\nu_{i} G_{i}^{(+1 / 2)}, M_{x}^{(0)}=x_{a} M_{a}^{(0)}, \\
\left\langle G_{i}^{(-1 / 2)}, G_{j}^{(+1 / 2)}\right\rangle & =-\left\langle G_{j}^{(-1 / 2)}, G_{i}^{(+1 / 2)}\right\rangle=\delta_{i j},\left\langle M_{a}^{(0)}, M_{b}^{(0)}\right\rangle=\left\langle M_{b}^{(0)}, M_{a}^{(0)}\right\rangle=\delta_{a b},
\end{aligned}
$$

where $G_{i}^{(-1 / 2)} \in \mathcal{M}_{F}^{(-1 / 2)}, G_{i}^{(+1 / 2)} \in \mathcal{M}_{F}^{(+1 / 2)}, M_{a}^{(0)} \in \mathcal{M}_{B}^{(0)}$ span the image part $\mathcal{M}$ of the algebra $\widehat{\mathfrak{f}}$ and $x$ and $\mu, \nu$ are even/odd constant parameters, respectively. Setting

$$
\psi_{+}^{(+1 / 2)}=\psi_{i} G_{i}^{(+1 / 2)}, \quad \psi_{-}^{(-1 / 2)}=\bar{\psi}_{i} G_{i}^{(-1 / 2)}, \quad A_{+}^{(0)}=A_{+a}^{(0)} M_{a}^{(0)}, \quad A_{-}^{(0)}=A_{-a}^{(0)} M_{a}^{(0)}
$$

and using $L_{+}, L_{-}^{\prime}$ in their original forms

$$
L_{+}=\partial_{+}+\left(A_{+}^{(0)}+Q_{+}^{(0)}+\psi_{+}^{(+1 / 2)}+\Lambda_{+}^{(+1)}\right), \quad L_{-}^{\prime}=\partial_{-}-\left(A_{-}^{(0)}+Q_{-}^{(0)}+\psi_{-}^{(-1 / 2)}+\Lambda_{-}^{(-1)}\right),
$$

we easily find the differentials associated to the dynamical fields

$$
\begin{aligned}
\mu \cdot \psi & =\left\langle G_{\mu}^{(-1 / 2)}, L_{+}\right\rangle \rightarrow d_{+}(\mu \cdot \psi)=G_{\mu}^{(-1 / 2)}, \\
\nu \cdot \bar{\psi} & =-\left\langle G_{\nu}^{(+1 / 2)}, L_{-}^{\prime}\right\rangle \rightarrow d_{-}^{\prime}(\nu \cdot \bar{\psi})=-G_{\nu}^{(+1 / 2)}, \\
x \cdot A_{+}^{(0)} & =\left\langle M_{x}^{(0)}, L_{+}\right\rangle \rightarrow d_{+}\left(x \cdot A_{+}^{(0)}\right)=M_{x}^{(0)}, \\
y \cdot A_{-}^{(0)} & =\left\langle M_{y}^{(0)}, L_{-}^{\prime}\right\rangle \rightarrow d_{-}^{\prime}\left(y \cdot A_{-}^{(0)}\right)=-M_{y}^{(0)} .
\end{aligned}
$$

The Poisson form of the supersymmetry flow transformations $\delta_{ \pm 1 / 2}$ of the field components is encoded in the following proposition.

Proposition 4 The supersymmetry transformations of the fields $\Phi(x)$ are Hamiltonian flows on the reduced phase space and are induced by the supercharges $Q^{ \pm}$in terms of the second Hamiltonian structure

$$
\rho \cdot \delta_{+1 / 2} \Phi(x)=-\left\{Q^{+}, \Phi(x)\right\}_{2}(L), \quad \lambda \cdot \delta_{-1 / 2} \Phi(x)=+\left\{Q^{-}, \Phi(x)\right\}_{2}\left(L^{\prime}\right),
$$

where $\rho, \lambda$ are constant even/odd parameters depending respectively, if the field $\Phi$ is even/odd. This definition is equivalent to the following matrix supersymmetry transformations

$$
\begin{aligned}
\left(\delta_{+1 / 2} B B^{-1}\right)^{\|} & =\left[\psi^{(-1 / 2)}, D^{(+1 / 2)}\right], \\
\delta_{+1 / 2} \psi_{+}^{(+1 / 2)} & =\left[\left(\partial_{+} B B^{-1}\right)^{\|}, D^{(+1 / 2)}\right], \\
\delta_{+1 / 2} \psi_{-}^{(-1 / 2)} & =-\left[\Lambda_{-}^{(-1)}, B^{-1} D^{(+1 / 2)} B\right],
\end{aligned}
$$

and

$$
\begin{aligned}
\left(B^{-1} \delta_{-1 / 2} B\right)^{\|} & =-\left[\psi^{(+1 / 2)}, D^{(-1 / 2)}\right], \\
\delta_{-1 / 2} \psi_{+}^{(+1 / 2)} & =\left[\Lambda_{+}^{(+1)}, B D^{(-1 / 2)} B^{-1}\right], \\
\delta_{-1 / 2} \psi_{-}^{(-1 / 2)} & =-\left[\left(B^{-1} \partial_{-} B\right)^{\|}, D^{(-1 / 2)}\right] .
\end{aligned}
$$

which should be compared with 45), 460) in the $A=\theta=0$ gauge. 
Proof. We will prove for the $\delta_{+1 / 2}$ supersymmetry flow variations as for the $\delta_{-1 / 2}$ the proof follows exactly the same lines. For the components $\psi$ in $\psi_{+}^{(+1 / 2)}=\psi_{i} G_{i}^{(+1 / 2)}$, we have (with $\mu$ fermionic) that

$$
\mu \cdot \delta_{+1 / 2} \psi=-\left\{m_{+}, \mu \cdot \psi\right\}_{2}\left(L_{+}\right),
$$

because $\psi$ and its differential vanishes when restricted to $L_{-}^{\prime}$. Using the explicit form of the Lax connection

$$
\Lambda_{\Theta}^{(+1)}=A_{+}^{(0)}+Q_{+}^{(0)}+\psi_{+}^{(+1 / 2)}+\Lambda_{+}^{(+1)},
$$

the $r$-matrix $\mathcal{R}_{<}=\frac{1}{2}\left(\mathcal{P}_{0}-\mathcal{P}_{<0}\right)$ and the first equation in (88), we conclude that there is not contribution from the derivative terms because $d_{+}(\mu \cdot \psi)$ has $Q$ grade $-1 / 2$. Then, the non-zero contribution to the bracket i. 24

$$
\left\{m_{+}, \mu \cdot \psi\right\}_{2}\left(L_{+}\right)=\left\langle\Lambda_{\Theta}^{(+1)},\left[d_{+} m_{+}, d_{+}(\mu \cdot \psi)\right]_{\mathcal{R}_{<}}\right\rangle=-\left\langle\Lambda_{+}^{(+1)},\left[\left(d_{+} m_{+}\right)_{-1 / 2}, G_{\mu}^{(-1 / 2)}\right]\right\rangle .
$$

The only contribution from the differential $\left(d_{+} m_{+}\right)_{-1 / 2}$ is the one along the image, which is given by $\left(d_{+} m_{+}\right)_{-1 / 2}^{\|}=$ $\left[\psi^{(-1)}, D^{(+1 / 2)}\right]$. Now, using the ad-invariance of the inner product, the Jacobi identity, the definition $A_{+}^{(0)}=\left[\psi^{(-1)}, \Lambda_{+}^{(+1)}\right]$ and (30) in the gauge $A=0$, we find that

$$
\mu \cdot \delta_{+1 / 2} \psi=\left\langle\left[\left(\partial_{+} B B^{-1}\right)^{\|}, D^{(+1 / 2)}\right], G_{\mu}^{(-1 / 2)}\right\rangle, \quad \delta_{+1 / 2} \psi_{+}^{(+1 / 2)}=\left[\left(\partial_{+} B B^{-1}\right)^{\|}, D^{(+1 / 2)}\right],
$$

where we have used $\mu \cdot \delta_{+1 / 2} \psi=\left\langle\delta_{+1 / 2} \psi_{+}^{(+1 / 2)}, G_{\mu}^{(-1 / 2)}\right\rangle$ in order to isolate the supermatrix variation $\delta_{+1 / 2} \psi_{+}^{(+1 / 2)}$.

Similarly, for the components $\bar{\psi}$ in $\psi_{-}^{(-1 / 2)}=\bar{\psi}_{i} G_{i}^{(-1 / 2)}$, we get by the same restriction argument applied this time to $\bar{\psi}$ on $L_{-}$, that

$$
\nu \cdot \delta_{+1 / 2} \bar{\psi}=\left\{m_{-}, \nu \cdot \bar{\psi}\right\}_{2}\left(L_{-}\right) .
$$

Using the explicit form of the Lax connection

$$
\Lambda_{\Pi}^{(-1)}=B\left(\psi_{-}^{(-1 / 2)}+\Lambda_{-}^{(-1)}\right) B^{-1},
$$

the projected components of the differential

$$
\left(d_{-} m_{-}\right)_{+1 / 2}=-D^{(+1 / 2)}, \quad d_{-}(\nu \cdot \bar{\psi})=-B G_{\nu}^{(+1 / 2)} B^{-1},
$$

the second line of (88) and noting that the derivative terms does not contribute, we obtain

$$
\left\{m_{-}, \nu \cdot \bar{\psi}\right\}_{2}\left(L_{-}\right)=\left\langle\Lambda_{\Pi}^{(-1)},\left[d_{-} m_{-}, d_{-}(\nu \cdot \bar{\psi})\right]_{\mathcal{R}_{>}}\right\rangle=\left\langle B \Lambda_{-}^{(-1)} B^{-1},\left[\left(d_{-} m_{-}\right)_{+1 / 2}, B G_{\nu}^{(+1 / 2)} B^{-1}\right]\right\rangle .
$$

From this, we easily obtain the variation

$$
\nu \cdot \delta_{+1 / 2} \bar{\psi}=-\left\langle\left[\Lambda_{-}^{(-1)}, B^{-1} D^{(+1 / 2)} B\right], G_{\nu}^{(+1 / 2)}\right\rangle, \quad \delta_{+1 / 2} \psi_{-}^{(-1 / 2)}=-\left[\Lambda_{-}^{(-1)}, B^{-1} D^{(+1 / 2)} B\right],
$$

where we have used the ad-invariance of the inner product and $\nu \cdot \delta_{+1 / 2} \bar{\psi}=\left\langle\delta_{+1 / 2} \psi_{-}^{(-1 / 2)}, G_{\nu}^{(+1 / 2)}\right\rangle$ to isolate $\delta_{+1 / 2} \psi_{-}^{(-1 / 2)}$.

The transformation for $B$ is going to be found in an indirect way through the transformation of the quantity $A_{+}^{(0)}$, which is more natural. From the restriction of $A_{+}^{(0)}$ to $L_{+}$we find (with $x$ bosonic) that

$$
x \cdot \delta_{+1 / 2} A_{+}^{(0)}=-\left\{m_{+}, x \cdot A_{+}^{(0)}\right\}_{2}\left(L_{+}\right) .
$$

\footnotetext{
${ }^{24}$ We have drop the integration of $\mu \cdot \psi(x)$ inside the bracket but it have to be taken into account always when considering quantities in which the derivative terms contribute.
} 
The differentials entering the bracket are

$$
\left(d_{+} m_{+}\right)_{0}=\left[\psi^{(-1 / 2)}, D^{(+1 / 2)}\right], \quad d_{+}\left(x \cdot A_{+}^{(0)}\right)=M_{x}^{(0)}
$$

and by using (88) once more we obtain the non-zero contributions to it

$$
\begin{aligned}
\left\{m_{+}, x \cdot A_{+}^{(0)}\right\}_{2}\left(L_{+}\right) & =\left\langle\Lambda_{\Theta}^{(+1)},\left[\left(d_{+} m_{+}\right)_{0}, d_{+}\left(x \cdot A_{+}^{(0)}\right)\right]\right\rangle+\left\langle\partial_{+}\left(d_{+} m_{+}\right)_{0}, d_{+}\left(x \cdot A_{+}^{(0)}\right)\right\rangle \\
& =\left\langle\left[Q_{+}^{(0)},\left[\psi^{(-1 / 2)}, D^{(+1 / 2)}\right]\right]+\partial_{+}\left[\psi^{(-1 / 2)}, D^{(+1 / 2)}\right], M_{x}^{(0)}\right\rangle
\end{aligned}
$$

implying that

$$
x \cdot \delta_{+1 / 2} A_{+}^{(0)}=-\left\langle\left[Q_{+}^{(0)},\left[\psi^{(-1 / 2)}, D^{(+1 / 2)}\right]\right]+\partial_{+}\left[\psi^{(-1 / 2)}, D^{(+1 / 2)}\right], M_{x}^{(0)}\right\rangle .
$$

Now, in order to get the variation of the Toda field $B$, we perform a $\delta_{+1 / 2}$ variation on the definition of $A_{+}^{(0)}$, i.e (30) in the $A=0$ gauge. This gives

$$
\delta_{+1 / 2} A_{+}^{(0)}=-\partial_{+}\left(\delta_{+1 / 2} B B^{-1}\right)^{\|}-\left[\left(\delta_{+1 / 2} B B^{-1}\right)^{\|},\left(\partial_{+} B B^{-1}\right)^{\perp}\right]-\left[\left(\delta_{+1 / 2} B B^{-1}\right)^{\perp},\left(\partial_{+} B B^{-1}\right)^{\|}\right] .
$$

Using (30), the last line of (37) and projecting the resulting expression with $M_{x}^{(0)}$, we obtain

$$
x \cdot \delta_{+1 / 2} A_{+}^{(0)}=-\left\langle\left[Q_{+}^{(0)},\left(\delta_{+1 / 2} B B^{-1}\right)^{\|}\right]+\partial_{+}\left(\delta_{+1 / 2} B B^{-1}\right)^{\|}, M_{x}^{(0)}\right\rangle-\left\langle\left[A_{+}^{(0)},\left(\delta_{+1 / 2} B B^{-1}\right)^{\perp}\right], M_{x}^{(0)}\right\rangle .
$$

Finally, by comparing (96) and (97) we conclude that

$$
\left(\delta_{+1 / 2} B B^{-1}\right)^{\|}=\left[\psi^{(-1 / 2)}, D^{(+1 / 2)}\right], \quad\left(\delta_{+1 / 2} B B^{-1}\right)^{\perp}=0 .
$$

Following exactly the same steps for the $\delta_{-1 / 2}$ variation we arrive to the final result.

At this point we have an aparent discrepancy between the transformations (94), (95) and (45), (46). One set is local while the other is not because of the presence of the $\theta^{( \pm 1 / 2)}$ terms (47). The first set do not preserve the constraints (38) while the second set do. The easiest way to explain this difference is to work out the Noether procedure on reverse 25 . We will do it only for the $\delta_{+1 / 2}$ variations, for the obvious reason.

Consider the arbitrary variation (54) with $\delta=\delta_{+1 / 2}$ and without gauge fields, i.e the action functional (48). Taking into account (57) and (61) we get

$$
\begin{aligned}
\frac{2 \pi}{k} \delta_{+1 / 2} S_{\text {Toda }}[B, \psi] & =\int_{\Sigma}\left\langle\left(\partial_{+} K_{-}^{(-1 / 2)}-\partial_{-} K_{+}^{(-1 / 2)}\right),\left(U_{-}^{-1} \delta_{+1 / 2} \Phi U_{-}\right)\right\rangle \\
& =\int_{\Sigma}\left\langle\left(\partial_{+} \widetilde{K}_{-}^{(-1 / 2)}-\partial_{-} \widetilde{K}_{+}^{(-1 / 2)}\right),\left(\widetilde{S}_{-}^{-1} U_{-}^{-1} \delta_{+1 / 2} \Phi U_{-} \widetilde{S}_{-}\right)\right\rangle,
\end{aligned}
$$

where $\delta_{+1 / 2} \Phi=\delta_{+1 / 2} B B^{-1}-B \delta_{+1 / 2} \psi^{(+1 / 2)} B^{-1}-\delta_{+1 / 2} \psi^{(-1 / 2)}$ and where we have to solve the relations

$$
\left(U_{-}^{-1} \delta_{+1 / 2} \Phi U_{-}\right)_{+1 / 2}^{\perp}=\left(\widetilde{S}_{-}^{-1} U_{-}^{-1} \delta_{+1 / 2} \Phi U_{-} \widetilde{S}_{-}\right)_{+1 / 2}^{\perp}=D^{(+1 / 2)} .
$$

Taking respectively, the following solutions

$$
\delta_{+1 / 2} \Phi=\left(U_{-} D^{(+1 / 2)} U_{-}^{-1}\right)_{\geq-1 / 2}=\left(U_{-} \widetilde{S}_{-} D^{(+1 / 2)} \widetilde{S}_{-}^{-1} U_{-}^{-1}\right)_{\geq-1 / 2}
$$

with $\widetilde{S}_{-}=\exp \left(\theta^{(-1 / 2)}+\theta^{(-1)}+\ldots\right), \theta^{(r)} \in \mathcal{K}$ and using (26) , we get the local form (94), (95)) corresponding to the local canonical DS supercharges (69) and the non-local form (45), (46) corresponding to the gauge transformation

\footnotetext{
${ }^{25}$ I want to thank Tim Hollowood for discussions on this point.
} 
of the supercharges (69), respectively. This shows that we can always perform a compensating gauge transformation $U_{ \pm} \rightarrow U_{ \pm} \widetilde{S}_{ \pm}$in order to preserve the constraints (38) and this selects the special value of $\theta^{( \pm 1 / 2)}$ to be (47). This means that the flows $\delta_{ \pm 1 / 2}$ are symmetry flows on the coadjoint orbits $L_{+}, L_{-}^{\prime}$.

Let us compute the algebra of the supersymmetry flows (94), (95) in the perturbative limit $B=1+b$. Using the fermionic equations of motion given by (37), we find, as expected from (90) that

$$
\left[\delta_{+1 / 2}, \delta_{+1 / 2}^{\prime}\right]=2 \epsilon \cdot \epsilon^{\prime} \partial_{+}, \quad\left[\delta_{-1 / 2}, \delta_{-1 / 2}^{\prime}\right]=2 \bar{\epsilon} \cdot \bar{\epsilon}^{\prime} \partial_{-}
$$

where we have used $\left[D^{(+1 / 2)}, D^{\prime(+1 / 2)}\right]=2 \epsilon \cdot \epsilon^{\prime} \Lambda_{+}^{(+1)},\left[D^{(-1 / 2)}, D^{\prime(-1 / 2)}\right]=-2 \bar{\epsilon} \cdot \bar{\epsilon}^{\prime} \Lambda_{-}^{(-1)}$, the jacobi identity and the relations (26). However, for the mixed terms we obtain

$$
\left[\delta_{+1 / 2}, \delta_{-1 / 2}\right]=\delta_{H}
$$

where $\delta_{H}=[\omega$,$] is a gauge transformation with parameter \omega=\left[D^{(+1 / 2)}, D^{(-1 / 2)}\right] \in \mathcal{K}_{B}^{(0)}$. In principle, for the mixed bracket we should obtain $\left\{Q^{+}, Q^{-}\right\}_{2} \sim Q_{H}$, where $Q_{H}$ is the Noether charge corresponding to the gauge group $H$, but the corresponding Noether current components $J^{\mu}$ are precisely the constraints (38) which vanishes identically. Then, the non-zero contribution to the conserved charge must come from the ambiguous term in the definition of a conserved current $\mathcal{J}^{\mu}=J^{\mu}+\varepsilon^{\mu \nu} \partial_{\nu} \mathcal{F}$, with $\mathcal{F}$ and arbitrary function. For this reason we have not writen the mixed bracket $\left\{Q^{+}, Q^{-}\right\}_{2}$ above. A more refined study of the supercharge algebra in terms of the subtracted monodromy 26 matrix will be done elsewhere [10. For the moment, we content ourselves with the result (90), but let us note that the perturbative excitations $b^{\|}, \psi_{ \pm}^{( \pm 1 / 2)}$ do transform under the kernel algebra $\mathcal{K}=\widehat{\mathfrak{f}}^{\perp}$ through the variations $\delta_{\mathcal{K}}$

Let us end this section by writing the brackets for the fundamental fields $B$ and $\psi^{( \pm 1 / 2)}$. To do this, it is more convenient to use (32) and (33) in the gauge $A=0$. Defining the currents $J_{+}=-\partial_{+} B B^{-1}, J_{-}=B^{-1} \partial_{-} B$, which have to be considered as functionals on $L_{+}$and $L_{-}^{\prime}$ respectively, and introducing a normalized basis for $\widehat{\mathfrak{f}}_{0}=\operatorname{span}\left(T_{a}^{(0)}\right)$ with $\left\langle T_{a}^{(0)}, T_{b}^{(0)}\right\rangle=\delta_{a b}$, we find the current differentials

$$
d\left(\lambda \cdot J_{+}\right)=T_{\lambda}^{(0)}, \quad d_{-}^{\prime}\left(\lambda \cdot J_{-}\right)=T_{\lambda}^{(0)},
$$

where $\lambda \cdot J_{ \pm}=\lambda_{a} J_{ \pm}^{a}, T_{\lambda}^{(0)}=\lambda_{a} T_{a}^{(0)}, a=1, \ldots, \operatorname{dim} \widehat{\mathfrak{f}}^{(0)}$ with $\lambda$ bosonic constants. Adding the fermionic field differentials (92) and using (87), (88) we find the brackets

$$
\begin{aligned}
\left\{\lambda \cdot J_{+}(x), \rho \cdot J_{+}(y)\right\}_{2}(L) & =\delta(x-y)\left\langle J_{+}(x),\left[T_{\lambda}^{(0)}, T_{\rho}^{(0)}\right]\right\rangle-\delta^{\prime}(x-y)\left\langle T_{\lambda}^{(0)}, T_{\rho}^{(0)}\right\rangle, \\
\{\mu \cdot \psi(x), \nu \cdot \psi(y)\}_{2}(L) & =-\mu \cdot a^{+} \cdot \nu \delta(x-y),
\end{aligned}
$$

where we have assumed that $\left[\Lambda_{+}^{(+1)}, G_{i}^{(-1 / 2)}\right]=a_{i j}^{+} G_{j}^{(+1 / 2)}$. For $J_{-}$and $\bar{\psi}$, the brackets in $L^{\prime}$ are quite similar but with $a_{i j}^{+} \rightarrow-a_{i j}^{-}$. Then, the second Hamiltonian structure is of Kac-Moody type 27 .

Finally, if we compute the canonical brackets for the action functional (48) we arrive to the conclusion that $\{X, Y\}_{2} \sim$ $\{X, Y\}_{W Z N W}$. It would be interesting to investigate more deeply the relation between the brackets $\{X, Y\}_{1,2}$ and the brackets $\{X, Y\}^{[ \pm 2],[0]}$ introduced in [18, which studies the connection between the Hamiltonian structures of the Green-Schwarz action and the generalized sine-Gordon models involved in the reduction of the $A d S_{5} \times S^{5}$ superstring sigma model. This will be done elsewhere [10].

\footnotetext{
${ }^{26}$ In [17] it was shown, by using monodromy matrix arguments, that $\mathcal{F}$ is unambiguously fixed and that the conserved charge $Q_{H}$ is of a kink type given by $B(+\infty) B(-\infty)^{-1}=\exp Q_{H}$.

${ }^{27}$ This was already noticed in [15] in the purely bosonic case. See the last example worked out in that reference.
} 


\section{Pohlmeyer reduction of GS superstring sigma models.}

In this chapter we briefly review the steps involved in the reduction of Green-Schwarz (GS) superstring sigma models on semi-symmetric superspaces. The aim is to show how emerges the extended homogeneous hierarchy integrable structure defined above and also to show the connection between the number of fermionic symmetry flows with the elements in the rank of the kappa symmetry.

A coset $F / G$ of a supergroup $F$ is a semi-symmetric superspace if it is invariant under a $\mathbb{Z}_{4}$ symmetry and the superalgebra admits the decomposition (1), $\mathfrak{f}=\mathfrak{f}_{0} \oplus \mathfrak{f}_{1} \oplus \mathfrak{f}_{2} \oplus \mathfrak{f}_{3}$, which is consistent with the relations $\left[\mathfrak{f}_{i}, \mathfrak{f}_{j}\right] \subset \mathfrak{f}_{(i+j)} \bmod 4$, the subspace $\mathfrak{f}_{j}$ is defined by $\Omega\left(\mathfrak{f}_{j}\right)=i^{j} \mathfrak{f}_{j}$ and the denominator subalgebra is the invariant subspace $\mathfrak{f}_{0}$.

We assume that the following decomposition of the bosonic subalgebra $\mathfrak{f}_{B}=\mathfrak{f}_{0} \oplus \mathfrak{f}_{2}$ also holds

$$
\begin{aligned}
\mathfrak{f}_{0} & =\mathfrak{m}_{0} \oplus \mathfrak{h}_{0}, \mathfrak{f}_{2}=\mathfrak{a}_{2} \oplus \mathfrak{n}_{2}, \quad\left[\mathfrak{a}_{2}, \mathfrak{a}_{2}\right]=0, \quad\left[\mathfrak{a}_{2}, \mathfrak{h}_{0}\right]=0, \quad\left[\mathfrak{h}_{0}, \mathfrak{h}_{0}\right] \subset \mathfrak{h}_{0}, \\
{\left[\mathfrak{m}_{0}, \mathfrak{m}_{0}\right] } & \subset \mathfrak{h}_{0}, \quad\left[\mathfrak{h}_{0}, \mathfrak{m}_{0}\right] \subset \mathfrak{m}_{0}, \quad\left[\mathfrak{m}_{0}, \mathfrak{a}_{2}\right] \subset \mathfrak{n}_{2}, \quad\left[\mathfrak{a}_{2}, \mathfrak{n}_{2}\right] \subset \mathfrak{m}_{0},
\end{aligned}
$$

where $\mathfrak{a}_{2}$ is a maximal Abelian subalgebra and $\mathfrak{h}_{0}$ its centralizer. The algebra $\mathfrak{h}_{0}$ turns out to be related to the gauge flows $H_{L} \times H_{R}$ introduced above.

The action of the sigma model can be written in terms of the $\mathbb{Z}_{4}$ decomposition of the current

$$
J_{\mu}=f^{-1} \partial_{\mu} f=\mathcal{A}_{\mu}+Q_{1 \mu}+P_{\mu}+Q_{3 \mu},
$$

where $\mathcal{A}_{\mu} \in \mathfrak{f}_{0}, Q_{1 \mu} \in \mathfrak{f}_{1}, P_{\mu} \in \mathfrak{f}_{2}, Q_{3 \mu} \in \mathfrak{f}_{3}$. The current $J$ is invariant under global left $F$-gauge transformations $f(x) \rightarrow f^{\prime} f(x)$ and under local right $G$-gauge transformations $f(x) \rightarrow f(x) g(x)$, the component $\mathcal{A}$ transform as a gauge connection, while the others components transform covariantly (in the adjoint).

The action of the non linear sigma model must be gauge invariant and $\mathbb{Z}_{4}$ symmetric and we are interested here in those models which are described by the following (Green-Schwarz) action

$$
S_{G S}=\frac{1}{2 \kappa^{2}} \int\left\langle\gamma^{\mu \nu} P_{\mu} P_{\nu}+\epsilon^{\mu \nu} Q_{1 \mu} Q_{3 \nu}\right\rangle,
$$

where $\gamma^{\mu \nu}=\sqrt{-g} g^{\mu \nu}, g_{\mu \nu}$ is the two dimensional world-sheet metric, $\epsilon_{\mu \nu}$ the anti-symmetric symbol and $\kappa$ is the sigma model coupling. The action (104) is invariant under 2D conformal transformations, $G$-gauge transformations and $\kappa$-symmetry, which is a local fermionic gauge transformation and all this means that some of the bosonic and fermionic degrees of freedom in the lagrangian (104) are un-physical.

The Pohlmeyer reduction consist of a classical removal of all the un-physical degrees of freedom and such a reduction is performed by gauge fixing all the above mentioned symmetries. We now briefly show how this is achieved, for full details see the original paper [1], see also [19].

Start by writing (104) in the conformal gaug 28

$$
\mathcal{L}_{G S}=\left\langle P_{+} P_{-}+\frac{1}{2}\left(Q_{1+} Q_{3-}-Q_{1-} Q_{3+}\right)\right\rangle .
$$

The classical field theory is describe by the equations of motion extracted from varying the action functional of (105)

$$
\begin{aligned}
0 & =\partial_{+} P_{-}+\left[\mathcal{A}_{+}, P_{-}\right]+\left[Q_{3+}, Q_{3-}\right], \\
0 & =\partial_{-} P_{+}+\left[\mathcal{A}_{-}, P_{+}\right]+\left[Q_{1-}, Q_{1+}\right], \\
0 & =\left[P_{+}, Q_{1-}\right]=\left[P_{-}, Q_{3+}\right]
\end{aligned}
$$

\footnotetext{
${ }^{28}$ We use here the light-cone convention $\gamma^{+-}=\gamma^{-+}=1$ and $\epsilon^{+-}=-\epsilon^{-+}=1$ of [1].
} 
and by the Maurer-Cartan identity for the flat current (103)

$$
\partial_{+} J_{-}-\partial_{-} J_{+}+\left[J_{+}, J_{-}\right]=0
$$

both supplemented with the Virasoro constraints

$$
\left\langle P_{+}, P_{+}\right\rangle=0, \quad\left\langle P_{-}, P_{-}\right\rangle=0 .
$$

Now, by using the polar decomposition theorem and the $G$-gauge freedom on the coset we can go to the so-called reduction gauge in which the components of the current $P_{\mu} \in \mathfrak{f}_{2}$ can be put in the following form

$$
P_{+}=\mu_{+}\left(x^{+}\right) \Lambda_{+}, \quad P_{-}=\mu_{-}\left(x^{-}\right) B \Lambda_{-} B^{-1},
$$

where $\Lambda_{ \pm} \in \mathfrak{a}_{2}$ are constant elements in $\mathfrak{f}, \mu_{ \pm}\left(x^{ \pm}\right)$are functions of $x^{ \pm}$only and $B \in G=\exp \mathfrak{f}_{0}$ belongs to the sigma model gauge group, i.e the denominator group in the coset $F / G$. For the semi-symmetric-space cosets of interest, we have that $\operatorname{dim} \mathfrak{a}_{2}=1$ meaning that $\Lambda_{+}=\Lambda_{-}=\Lambda$ is unique. We use this constant element $\Lambda$, which is also semisimple, to introduce the $\mathbb{Z}_{2}$ superalgebra decomposition (2) with $\mathfrak{f}=\mathfrak{f}^{\perp} \oplus \mathfrak{f}^{\|}$.

The $\kappa$-symmetry can be partially fixed by the gauge condition $Q_{1-}=0$ and $Q_{3+}=0$ and this simplifies the equations (106). Replacing (109) into (106), using (108) and the residual conformal transformations we can set the functions $\mu_{ \pm}\left(x^{ \pm}\right)$to constants $\mu_{ \pm}$. The remaining equations of motion in (106) are equations for the gauge field components $\mathcal{A}_{ \pm}$only and allow the following solution

$$
\mathcal{A}_{+}=-\left(\partial_{+} B B^{-1}+B A_{+}^{(R)} B^{-1}\right), \quad \mathcal{A}_{-}^{\perp}=-A_{-}^{(L)}, \quad \mathcal{A}_{-}^{\|}=0,
$$

where $A_{+}^{(R)}, A_{-}^{(L)} \in \mathfrak{f}_{0}^{\perp}=\mathfrak{h}_{0}$ belong now to a subalgebra of the former sigma model gauge algebra $\mathfrak{f}_{0}$, see(102). All this exhaust the equations of motion in (106).

The solutions of the Virasoro constraints (108) are now 29

$$
P_{+}=\mu_{+} \Lambda_{+}, \quad P_{-}=\mu_{-} B \Lambda_{-} B^{-1}
$$

and the gauge group that preserves these solutions and the Virasoro "surface" (108) is precisely the $H_{L} \times H_{R}$ gauge symmetry introduced above, $\widetilde{B}=\Gamma_{L} B \Gamma_{R}$. This explains the use of the notation $L, R$.

In terms of the new bosonic field variables $A_{+}^{(R)}, A_{-}^{(L)} \in \mathfrak{f}_{0}^{\perp}, B \in G$ and in the gauge $Q_{1-}=0, Q_{3+}=0$, the (remaining) Maurer-Cartan equations (107) can be put in the form

$$
\begin{aligned}
D_{-}^{(L)} Q_{1+} & =\left[\Lambda_{+}, Q_{3-}\right] \\
D_{-}^{(L)}\left(\partial_{+} B B^{-1}+B A_{+}^{(R)} B^{-1}\right)-\partial_{+} A_{-}^{(L)} & =-\left[\Lambda_{+}, B \Lambda_{+} B^{-1}\right]-\left[Q_{1+}, Q_{3-}\right], \\
D_{+}^{(R)}\left(B^{-1} Q_{3-} B\right) & =\left[\Lambda_{-}, B^{-1} Q_{1+} B\right],
\end{aligned}
$$

where $D_{-}^{(L)}=\partial_{-}-\left[A_{-}^{(L)},\right], D_{+}^{(R)}=\partial_{+}-\left[A_{+}^{(R)},\right]$ are the covariant derivatives for the $H_{L} \times H_{R}$ action of the gauge group and $\widetilde{Q}_{1+}=\Gamma_{L} Q_{1+} \Gamma_{L}^{-1}, \widetilde{Q}_{3-}=\Gamma_{L} Q_{3-} \Gamma_{L}^{-1}$ are the gauge transformations for the remaining fermionic current components. At this point it is worth it to compare these equations with (35).

We now make the following change of field variables $Q_{1+}, Q_{3-} \rightarrow \Psi_{1}, \Psi_{3}$ defined by

$$
\Psi_{1}=Q_{1+}, \quad \Psi_{3}=-B^{-1} Q_{3-} B .
$$

\footnotetext{
${ }^{29}$ From now we normalize $\mu_{ \pm} \rightarrow 1$.
} 
The $\kappa$-symmetry is completely fixed by putting to zero all the components of $\Psi_{1}, \Psi_{3}$ belonging to the fermionic part of kernel, i.e $\mathfrak{f}_{1}^{\perp}, \mathfrak{f}_{3}^{\perp}$. The remaining components in the fermionic part in the Image, i.e $\mathfrak{f}_{1}^{\|}, \mathfrak{f}_{3}^{\|}$are the truly fermionic physical degrees of freedom

$$
\Psi_{L}=\Psi_{1}^{\|}, \quad \Psi_{R}=\Psi_{3}^{\|}
$$

In terms of the Pohlmeyer reduced model field content $A_{+}^{(R)}, A_{-}^{(L)}, B, \Psi_{R}$ and $\Psi_{L}$, the Maurer-Cartan equations (111) can be written, with the help of a spectral parameter $z$, as the compatibility condition of the following Lax pair

$$
\begin{aligned}
& L_{+}(A)=\partial_{+}-\partial_{+} B B^{-1}-B A_{+}^{(R)} B^{-1}+\left(z^{+1 / 2} \Psi_{L}\right)+\left(z \Lambda_{+}\right), \\
& L_{-}(A)=\partial_{-}-A_{-}^{(L)}-B\left[\left(z^{-1 / 2} \Psi_{R}\right)+\left(-z^{-1} \Lambda_{-}\right)\right] B^{-1} .
\end{aligned}
$$

Making the following identifications

$$
\begin{aligned}
\left(z^{+1 / 2} \Psi_{R}\right) & =\psi_{+}^{(+1 / 2)}, & \left(z \Lambda_{+}\right) & =\Lambda_{+}^{(+1)}, \\
\left(z^{-1 / 2} \Psi_{L}\right) & =\psi_{-}^{(-1 / 2)}, & \left(-z^{-1} \Lambda_{-}\right) & =\Lambda_{-}^{(-1)}
\end{aligned}
$$

and comparing with (32) we see that the Lax operators (113) and (55) describe the same integrable hierarchy (the extended homogeneous hierarchy). From (3) we see that the fermionic identifications are consistent

$$
\left(z^{+1 / 2} \Psi_{L}\right)=\left[\psi^{(-1 / 2)}, \Lambda_{+}^{(+1)}\right], \quad\left(z^{-1 / 2} \Psi_{R}\right)=-\left[\psi^{(+1 / 2)}, \Lambda_{-}^{(-1)}\right]
$$

because $\Psi_{L} \in \mathfrak{f}_{1}^{\|}, \psi^{(-1 / 2)} \in \widehat{\mathfrak{f}}_{3}^{\|}$and $\Psi_{R} \in \mathfrak{f}_{3}^{\|}, \psi^{(+1 / 2)} \in \widehat{\mathfrak{f}}_{1}^{\|}$. Recall that $\operatorname{ad}(\Lambda)$ maps the subspaces $\mathfrak{f}_{1,3} \rightarrow \mathfrak{f}_{3,1}$ each other. To obtain the equivalent formulation $L_{ \pm}^{\prime}(A)$ we use the $B$-conjugated solution of (110).

These equations are invariant under the gauge group $H_{L} \times H_{R}$ and all the results found above also applies here. Note that $\mathfrak{f}_{0}^{\perp}=\mathfrak{h}_{0} \subset \operatorname{ker}(a d(\Lambda))$ generates gauge flows associated to grade zero elements after embedding $\mathfrak{f}$ into the loop algebra $\widehat{\mathfrak{f}}$ in the form (3). The Pohlmeyer reduction in now clear. It states that (104) and (52) describes the same classical field theory30. Note that the net effect of the reduction is to trade the Euler-Lagrange equations (106) of (104) by the Maurer-Cartan identities (107) with associated Lax Pair (113), which correspond now to the Euler-Lagrange equations of the SSSSG action (52). The local gauge symmetry is reduced from the right $G$-gauge action on the coset $F / G$ of the GS sigma model to the left-right $H$-gauge action on the coset $G / H$ of the gauged WZNW model coupled to fermions and the fields which include the un-physical degrees of freedom are reduced from $P_{\mu}, Q_{1 \mu}, Q_{3 \mu}$ to $B, \psi_{ \pm}^{( \pm 1 / 2)}, A_{ \pm}^{(R / L)}$, which have physical degrees of freedom only. Concerning the local $\kappa$-symmetry, it seems to be that its global remnant is related to the existence of $2 \mathrm{D}$ world-sheet extended supersymmetry in the reduced models. We do not have a formal proof of this statement but we will provide some evidence that this is the case because, as discussed above, the elements in the fermionic kernel $\mathcal{K}_{F}^{( \pm 1 / 2)}$ generate $\operatorname{dim} \mathcal{K}_{F}^{( \pm 1 / 2)}$ global symmetry flows with conserved supercharges (68). Note that physical fields are parametrized in $\mathcal{M}$ while the symmetries are associated to the sub-superalgebra $\mathcal{K} \subset \widehat{\mathfrak{f}}$ and we can see now the role of the dressing flow equations (16), (17): they generate global world-sheet symmetries $\delta_{\mathcal{K}}$ in the reduced model from the loop algebra $\mathcal{K}$ constructed out of the subalgebra $\mathfrak{f}^{\perp} \subset \mathfrak{f}$ of the global target space symmetry of the sigma model.

We consider now the rank of the $\kappa$-symmetry. Following [20], it is defined to be the number of fermionic generators in $\mathfrak{f}_{1}$ and $\mathfrak{f}_{3}$ which are annihilated by the adjoint action of $P_{ \pm} \in \mathfrak{f}_{2}$

$$
N_{\kappa}=\operatorname{dim} \operatorname{ker}\left(\operatorname{ad}\left(P_{-}\right) \mid \mathfrak{f}_{3}\right), \quad N_{\widetilde{\kappa}}=\operatorname{dim} \operatorname{ker}\left(\operatorname{ad}\left(P_{+}\right) \mid \mathfrak{f}_{1}\right)
$$

with $P_{ \pm}$satisfying the Virasoro constraints (108). We assume that we are in the situation of generic classical solutions which is when the number of zero modes $N_{\kappa}, N_{\widetilde{\kappa}}$ are field (i.e $P_{ \pm}$) independent and are determined entirely by the

\footnotetext{
${ }^{30}$ The action (52) is slightly different than the one originally constructed in [1] in the particular case of the $A d S_{5} \times S^{5}$ superstring sigma model, the difference is in the potential term and it is not essential.
} 
Lie superalgebra properties of the algebra $\mathfrak{f}$ of interest. This means that we can use $a d\left(\Lambda_{ \pm}\right)$instead of $\operatorname{ad}\left(P_{ \pm}\right)$to compute the dimensions (114). In [20 the dimensions (114) were calculated for several Lie superalgebras admitting the $\mathbb{Z}_{4}$ decomposition (1). Here we write some of those dimensions of our interest

$$
\begin{aligned}
A d S_{2} \times S^{2} & \rightarrow N_{\kappa}=N_{\widetilde{\kappa}}=2, \\
A d S_{3} \times S^{3} & \rightarrow N_{\kappa}=N_{\widetilde{\kappa}}=4, \\
A d S_{4} \times \mathbb{C} P^{3} & \rightarrow \quad N_{\kappa}=N_{\widetilde{\kappa}}=4, \\
A d S_{5} \times S^{5} & \rightarrow \quad N_{\kappa}=N_{\widetilde{\kappa}}=8 .
\end{aligned}
$$

These are exactly the number of supersymmetry flows generated by the supercharges (68). In our notation and for the first two models in the list we have $\operatorname{dim} \mathcal{K}_{F}^{( \pm 1 / 2)}=2$ and $\operatorname{dim} \mathcal{K}_{F}^{( \pm 1 / 2)}=4$, respectively. See (143), (145), (149) and (151) for the explicit expressions of the generators in $\mathcal{K}_{F}$.

The $A d S_{5} \times S^{5}$ case is treated in detail in [9, where it is shown that the symmetry algebra of the solitonic spectrum of the reduced model, in semi-classical quantization, is precisely the kernel algebra $\mathcal{K}$, which in this case turns out to be isomorphic to a centrally extended $s u(2 \mid 2)^{\times 2}$ superalgebra. This algebra has $8+8$ fermionic elements in its odd part generating 16 supersymmetry flows and 12 bosonic elements in its even part generating the gauge algebra $s u(2)^{\times 4}$. A similar set of conserved supercharges (68) and supersymmetry transformations (45), (46) in the on-shell $A_{ \pm}=0$ gauge are also constructed for this case.

\section{Examples: supercharges from superspace and from symmetry flows.}

Because of the supersymmetry we are dealing with is quite non-standard, it is important to study its relation with the usual supersymmetry obtained from superspace by working out some examples. Here we consider the Pohlmeyer reduction of the superstring on $A d S_{2} \times S^{2}$ and $A d S_{3} \times S^{3}$ which are already known. As we are mainly interested in understanding the role of the supercharges found above, we will try to be as close as possible to the superspace results. However, we have to mention that in the $A d S_{3} \times S^{3}$ we will ignore the Wilson lines appearing in (68) and make the computations in some field limits.

\subsection{Supercharges of the Landau-Ginzburg models.}

Notation 5 In this section, the light-cone notation used is $x^{ \pm}=x^{0} \pm x^{1}, \partial_{ \pm}=\frac{1}{2}\left(\partial_{0} \pm \partial_{1}\right), \eta_{+-}=\eta_{-+}=-\frac{1}{2}, \eta^{+-}=$ $\eta^{-+}=-2$, corresponding to the metric $\eta_{00}=-1, \eta_{11}=+1$.

A Landau-Ginzburg model is defined by a Lagrangian density of the from (e.g see [21)

$$
\mathcal{L}=\int d^{4} \theta K\left(\Phi^{i}, \bar{\Phi}^{\bar{i}}\right)+\frac{1}{2}\left(\int d^{2} \theta W\left(\Phi^{i}\right)+c . c\right), i, \bar{i}=1, \ldots, n,
$$

where $\Phi=\phi\left(y^{ \pm}\right)+\theta^{\alpha} \psi_{\alpha}\left(y^{ \pm}\right)+\theta^{+} \theta^{-} F\left(y^{ \pm}\right)$is a chiral superfield, $y^{ \pm}=x^{ \pm}-i \theta^{ \pm} \bar{\theta}^{ \pm}, \bar{\theta}^{ \pm} \equiv\left(\theta^{ \pm}\right)^{*}$ and $\left(\psi_{1} \psi_{2}\right)^{*} \equiv\left(\psi_{2}^{*} \psi_{1}^{*}\right)$ stands for the complex conjugation convention acting on fermions.

In components, the lagrangian density is

$$
\begin{aligned}
\mathcal{L}= & -g_{i \bar{j}} \partial^{\mu} \phi^{i} \partial_{\mu} \bar{\phi}^{\bar{j}}+2 i g_{i \bar{j}} \bar{\psi}_{-}^{\bar{j}} D_{+} \psi_{-}^{i}+2 i g_{i \bar{j}} \bar{\psi}_{+}^{\bar{j}} D_{-} \psi_{+}^{i}+R_{i \bar{j} k \bar{l}} \psi_{+}^{i} \psi_{-}^{k} \bar{\psi}_{-}^{\bar{j}} \bar{\psi}_{+}^{\bar{l}}- \\
& -\frac{1}{4} g^{\bar{i} j} \partial_{\bar{i}} \bar{W} \partial_{j} W-\frac{1}{2} D_{i} \partial_{j} W \psi_{+}^{i} \psi_{-}^{j}-\frac{1}{2} D_{\bar{i}} \partial \overline{\bar{j}} \bar{W} \bar{\psi}_{-}^{\bar{i}} \bar{\psi}_{+}^{\bar{j}},
\end{aligned}
$$


where $g_{i \bar{j}}=\partial_{i} \partial_{\bar{j}} K\left(\phi^{i}, \bar{\phi}^{\bar{i}}\right), K$ is the Kähler potential and $D_{\mu} \psi_{ \pm}^{i}=\partial_{\mu} \psi_{ \pm}^{i}+\partial_{\mu} \phi^{j} \Gamma_{j k}^{i} \psi_{ \pm}^{k}, D_{i} \partial_{j} W=\partial_{i} \partial_{j} W-\Gamma_{i j}^{k} \partial_{k} W$. The $(2,2)$ Noether supercharges associated to the model (117) are given by

$$
Q_{ \pm}=\int_{-\infty}^{+\infty} d x^{1} G_{ \pm}^{0}, \quad G_{ \pm}^{0}=2 g_{i \bar{j}} \partial_{ \pm} \bar{\phi}^{\bar{j}} \psi_{ \pm}^{i} \mp \frac{i}{2} \bar{\psi}_{\mp}^{\bar{i}} \partial_{\bar{i}} \bar{W}
$$

\subsubsection{The $(2,2)$ sine-Gordon model.}

The first model of interest is when $i=1, K\left(\Phi^{1}, \bar{\Phi}^{\overline{1}}\right)=\bar{\Phi} \Phi$ and $W \rightarrow 2 W$ in the Lagrangian (117). In this case we have

$$
\mathcal{L}=2\left(\partial_{+} \varrho \partial_{-} \bar{\varrho}+\partial_{-} \varrho \partial_{+} \bar{\varrho}\right)+2 i \bar{\psi}_{-} \partial_{+} \psi_{-}+2 i \bar{\psi}_{+} \partial_{-} \psi_{+}-\left|W^{\prime}(\varrho)\right|^{2}-\left[W^{\prime \prime}(\varrho) \psi_{+} \psi_{-}+\bar{W}^{\prime \prime}(\bar{\varrho}) \bar{\psi}_{-} \bar{\psi}_{+}\right],
$$

where we have denoted by $\varrho$ the complex scalar field component of the superfield $\Phi$. The densities are

$$
G_{ \pm}^{0}=2 \partial_{ \pm} \bar{\varrho} \psi_{ \pm} \mp i \bar{\psi}_{\mp} \bar{W}^{\prime}(\bar{\varrho})
$$

Taking now the following choice of field components

$$
\varrho=\varphi+i \phi, \quad \psi_{-}=\lambda_{1}+i \lambda_{2}, \quad \psi_{+}=\bar{\lambda}_{1}+i \bar{\lambda}_{2}, \quad W(\varrho)=2 \mu \cos \varrho
$$

we have from (118), the $N=(2,2)$ supersymmetric extension of the sine-Gordon model [22]

$$
\begin{aligned}
\frac{1}{4} \mathcal{L}= & \partial_{+} \phi \partial_{-} \phi+\partial_{+} \varphi \partial_{-} \varphi+\frac{i}{2}\left(\lambda_{1} \partial_{-} \lambda_{1}+\lambda_{2} \partial_{-} \lambda_{2}+\bar{\lambda}_{1} \partial_{+} \bar{\lambda}_{1}+\bar{\lambda}_{2} \partial_{+} \bar{\lambda}_{2}\right)-\frac{\mu^{2}}{2}(\cosh 2 \phi-\cos 2 \varphi)+ \\
& +\mu i\left\{\cos \varphi \cosh \phi\left(\lambda_{1} \bar{\lambda}_{2}+\lambda_{2} \bar{\lambda}_{1}\right)-\sin \varphi \sinh \phi\left(\lambda_{1} \bar{\lambda}_{1}-\lambda_{2} \bar{\lambda}_{2}\right)\right\} .
\end{aligned}
$$

The fermionic densities (119) can be written as $G_{ \pm}^{0}=2\left(q_{1}^{ \pm}+i q_{2}^{ \pm}\right)$in terms of the following $2+2$ real component 31

$$
\begin{aligned}
{ }^{S S} q_{1}^{+} & =\lambda_{1} \partial_{+} \varphi+\lambda_{2} \partial_{+} \phi+\mu\left(\bar{\lambda}_{1} \cosh \phi \sin \varphi+\bar{\lambda}_{2} \sinh \phi \cos \varphi\right), \\
{ }^{S S} q_{2}^{+} & =-\lambda_{1} \partial_{+} \phi+\lambda_{2} \partial_{+} \varphi+\mu\left(-\bar{\lambda}_{1} \sinh \phi \cos \varphi+\bar{\lambda}_{2} \cosh \phi \sin \varphi\right), \\
{ }^{S S} q_{1}^{-} & =\bar{\lambda}_{1} \partial_{-} \varphi+\bar{\lambda}_{2} \partial_{-} \phi-\mu\left(\lambda_{1} \cosh \phi \sin \varphi+\lambda_{2} \sinh \phi \cos \varphi\right), \\
{ }^{S S} q_{2}^{-} & =-\bar{\lambda}_{1} \partial_{-} \phi+\bar{\lambda}_{2} \partial_{-} \varphi+\mu\left(\lambda_{1} \sinh \phi \cos \varphi-\lambda_{2} \cosh \phi \sin \varphi\right) .
\end{aligned}
$$

\subsubsection{The $(2,2)$ complex sine-Gordon and its hyperbolic counterpart.}

The second models of interest are when $i=1$ in the "compact" and "non-compact" cases, which are possibly related to two different truncations of the Pohlmeyer reduced $A d S_{3} \times S^{3}$ superstring. For the compact and non-compact models we choose, respectively, the following superfields components

$$
\begin{aligned}
\phi^{1} & =\ln \cos \varphi+i \theta, & \phi^{1} & =\ln \cosh \phi+i \chi \\
\psi_{ \pm}^{1} & =\tan \varphi e^{-i \theta} \chi_{ \pm}^{1}, & \psi_{ \pm}^{1} & =\tanh \phi e^{-i \chi} \rho_{ \pm}^{1}, \\
W\left(\phi^{1}\right) & =4 \mu e^{\phi^{1}}, & W\left(\phi^{1}\right) & =4 \mu e^{\phi^{1}},
\end{aligned}
$$

where $32 \chi_{+}^{1}=\left(\lambda_{1}+i \lambda_{2}\right), \chi_{-}^{1}=\left(\bar{\lambda}_{1}+i \bar{\lambda}_{2}\right), \rho_{+}^{1}=\left(\lambda_{3}+i \lambda_{4}\right)$ and $\rho_{-}^{1}=\left(\bar{\lambda}_{3}+i \bar{\lambda}_{4}\right)$.

\footnotetext{
${ }^{31}$ SS means Super-Space.

${ }^{32}$ The bar over the real fermion components should not be confused with the complex conjugation which is denoted by the same symbol.
} 
The kahler potentials are chosen such that

$$
g_{1 \overline{1}}=-\frac{1}{1-\left|e^{-\phi^{1}}\right|^{2}}=\cot ^{2} \varphi, \quad g_{1 \overline{1}}=\frac{1}{1-\left|e^{-\phi^{1}}\right|^{2}}=\operatorname{coth}^{2} \phi
$$

which imply

$$
\Gamma_{11}^{1}=\frac{1}{\sin ^{2} \varphi}, \quad R_{1 \overline{1} 1 \overline{1}}=\frac{\cot ^{4} \varphi}{\sin ^{2} \varphi} \text { and } \Gamma_{11}^{1}=-\frac{1}{\sinh ^{2} \phi}, \quad R_{1 \overline{1} 1 \overline{1}}=-\frac{\operatorname{coth}^{4} \phi}{\sinh ^{2} \phi}
$$

respectively.

In the real variables, the Lagrangian density (117) is (see also [23])

$$
\begin{aligned}
\frac{1}{4} \mathcal{L}= & \partial_{+} \varphi \partial_{-} \varphi+\cot ^{2} \varphi \partial_{+} \theta \partial_{-} \theta+\frac{i}{2}\left(\lambda_{1} \partial_{-} \lambda_{1}+\lambda_{2} \partial_{-} \lambda_{2}+\bar{\lambda}_{1} \partial_{+} \bar{\lambda}_{1}+\bar{\lambda}_{2} \partial_{+} \bar{\lambda}_{2}\right)+ \\
& +i \cot ^{2} \varphi\left[\partial_{-} \theta \lambda_{1} \lambda_{2}+\partial_{+} \theta \bar{\lambda}_{1} \bar{\lambda}_{2}\right]+\frac{1}{\sin ^{2} \varphi} \lambda_{1} \lambda_{2} \bar{\lambda}_{1} \bar{\lambda}_{2}- \\
& -\mu^{2} \sin ^{2} \varphi+\mu i \cos \varphi\left[\cos \theta\left(\lambda_{1} \bar{\lambda}_{2}+\lambda_{2} \bar{\lambda}_{1}\right)-\sin \theta\left(\lambda_{1} \bar{\lambda}_{1}-\lambda_{2} \bar{\lambda}_{2}\right)\right] .
\end{aligned}
$$

A similar lagrangian for $\mu=0$ was constructed in 24] by using a conventional (Kazama-Suzuki) gauged super WZNW model.

The supercharge densities can be expressed as $G_{ \pm}^{0}=2\left(q_{1}^{ \pm}+i q_{2}^{ \pm}\right)$in terms of the following $2+2$ real components

$$
\begin{aligned}
{ }^{S S} q_{1}^{+} & =\frac{1}{Y_{3}}\left(\lambda_{1} \partial_{+} Y_{1}+\lambda_{2} \partial_{+} Y_{2}\right)-\mu \bar{\lambda}_{2} Y_{3}, \\
{ }^{S S} q_{2}^{+} & =\frac{1}{Y_{3}}\left(-\lambda_{1} \partial_{+} Y_{2}+\lambda_{2} \partial_{+} Y_{1}\right)-\mu \bar{\lambda}_{1} Y_{3}, \\
{ }^{S S} q_{1}^{-} & =\frac{1}{Y_{3}}\left(\bar{\lambda}_{1} \partial_{-} Y_{1}+\bar{\lambda}_{2} \partial_{-} Y_{2}\right)+\mu \lambda_{2} Y_{3}, \\
{ }^{S S} q_{2}^{-} & =\frac{1}{Y_{3}}\left(-\bar{\lambda}_{1} \partial_{-} Y_{2}+\bar{\lambda}_{2} \partial_{-} Y_{1}\right)+\mu \lambda_{1} Y_{3},
\end{aligned}
$$

where $Y_{1}=\cos \theta \cos \varphi, Y_{2}=\sin \theta \cos \varphi$ and $Y_{3}=\sin \varphi$.

Similarly, for the non-compact model we have the Lagrangian density

$$
\begin{aligned}
\frac{1}{4} \mathcal{L}= & \partial_{+} \phi \partial_{-} \phi+\operatorname{coth}^{2} \phi \partial_{+} \chi \partial_{-} \chi+\frac{i}{2}\left(\lambda_{3} \partial_{-} \lambda_{3}+\lambda_{4} \partial_{-} \lambda_{4}+\bar{\lambda}_{3} \partial_{+} \bar{\lambda}_{3}+\bar{\lambda}_{4} \partial_{+} \bar{\lambda}_{4}\right)+ \\
& +i \operatorname{coth}^{2} \phi\left[\partial_{-} \chi \lambda_{3} \lambda_{4}+\partial_{+} \chi \bar{\lambda}_{3} \bar{\lambda}_{4}\right]+\frac{1}{\sinh ^{2} \phi} \lambda_{3} \lambda_{4} \bar{\lambda}_{3} \bar{\lambda}_{4}- \\
& -\mu^{2} \sinh ^{2} \phi-\mu i \cosh \phi\left[\cos \chi\left(\lambda_{3} \bar{\lambda}_{4}+\lambda_{4} \bar{\lambda}_{3}\right)-\sin \chi\left(\lambda_{3} \bar{\lambda}_{3}-\lambda_{4} \bar{\lambda}_{4}\right)\right]
\end{aligned}
$$

and the supercharge densities $G_{ \pm}^{0}=2\left(q_{1}^{ \pm}+i q_{2}^{ \pm}\right)$written in terms of the following $2+2$ real components

$$
\begin{aligned}
{ }^{S S} q_{1}^{+} & =\frac{1}{X_{3}}\left(\lambda_{3} \partial_{+} X_{1}+\lambda_{4} \partial_{+} X_{2}\right)-\mu \bar{\lambda}_{4} X_{3}, \\
{ }^{S S} q_{2}^{+} & =\frac{1}{X_{3}}\left(-\lambda_{3} \partial_{+} X_{2}+\lambda_{4} \partial_{+} X_{1}\right)-\mu \bar{\lambda}_{3} X_{3}, \\
{ }^{S S} q_{1}^{-} & =\frac{1}{X_{3}}\left(\bar{\lambda}_{3} \partial_{-} X_{1}+\bar{\lambda}_{4} \partial_{-} X_{2}\right)+\mu \lambda_{4} X_{3}, \\
{ }^{S S} q_{2}^{-} & =\frac{1}{X_{3}}\left(-\bar{\lambda}_{3} \partial_{-} X_{2}+\bar{\lambda}_{4} \partial_{-} X_{1}\right)+\mu \lambda_{3} X_{3},
\end{aligned}
$$

where $X_{1}=\cos \chi \cosh \phi, X_{2}=\sin \chi \cosh \phi$ and $X_{3}=\sinh \phi$.

Now, we proceed to use our formulation. 


\subsection{Supercharges of the Pohlmeyer reduced models.}

As mentioned above, the aim is to try to relate the superspace expressions (121), (123) and (125) with the supersymmetry flow result (68).

\subsubsection{Reduction of the $A d S_{2} \times S^{2}$ superstring and (2,2) 2D SUSY.}

This is the only known case in which a reduced model posses 2D world-sheet supersymmetry [1. However, the supersymmetry of this $A d S_{2} \times S^{2}$ reduced model was identified through its superspace description (120), i.e the $N=2$ supersymmetric sine-Gordon model. Here we use our general flow approach to confirm this fact from a different point of view.

From the general discussion we identify $\frac{F}{G}=\frac{P S U(1,1 \mid 2)}{U(1) \times U(1)}$ and $\frac{G}{H}=G$ because $H=\oslash$, as can be seen from (142). Then, this model has no gauge symmetries $\mathcal{K}_{B}^{(0)}=\oslash$ and from (147) we expect to obtain a reduced model with an ordinary extended $(2,2)$ supersymmetry. Fortunately, in this case we do note have gauge fields and Wilson lines and this means that the variations $\delta_{ \pm 1 / 2}$ can be lifted easily to the Lagrangian level (48). Note also that we do not have to deal with the constraints (38).

Using the basis (146), we parametrize the physical fields as

$$
B=\operatorname{diag}\left(B_{A}, B_{S}\right), \quad \psi^{(-1 / 2)}=\psi_{i} G_{i}^{(-1 / 2)}, \quad \psi^{(+1 / 2)}=\bar{\psi}_{i} G_{i}^{(+1 / 2)},
$$

where $i=1,2$ and $B_{A}, B_{S}$ are given by 33

$$
\begin{aligned}
& B_{A}=\exp \left(\phi M_{1}^{(0)}\right)=\left(\begin{array}{cc}
\cosh \phi & \sinh \phi \\
\sinh \phi & \cosh \phi
\end{array}\right) \in U(1) \\
& B_{S}=\exp \left(\varphi M_{2}^{(0)}\right)=\left(\begin{array}{cc}
\cos \varphi & i \sin \varphi \\
i \sin \varphi & \cos \varphi
\end{array}\right) \in U(1) .
\end{aligned}
$$

With $\Lambda$ defined in (144), we can compute all the terms entering the action (48). They are

$$
\begin{aligned}
\left\langle B^{-1} \partial_{+} B B^{-1} \partial_{-} B\right\rangle & =2\left(\partial_{+} \phi \partial_{-} \phi+\partial_{+} \varphi \partial_{-} \varphi\right) \\
\left\langle\psi_{+}^{(+1 / 2)} \partial_{-} \psi^{(-1 / 2)}+\psi_{-}^{(-1 / 2)} \partial_{+} \psi^{(+1 / 2)}\right\rangle & =2\left(\psi_{i} \partial_{-} \psi_{i}+\bar{\psi}_{i} \partial_{+} \bar{\psi}_{i}\right) \\
\mu^{2}\left\langle\Lambda_{+}^{(+1)} B \Lambda_{-}^{(-1)} B^{-1}\right\rangle & =-\frac{\mu^{2}}{2}(\cos 2 \varphi-\cosh 2 \phi) \\
\mu\left\langle\psi_{+}^{(+1 / 2)} B \psi_{-}^{(-1 / 2)} B^{-1}\right\rangle & =-2 \mu\left\{\cos \varphi \cosh \phi\left(\psi_{1} \bar{\psi}_{1}+\psi_{2} \bar{\psi}_{2}\right)+\sin \varphi \sinh \phi\left(\psi_{2} \bar{\psi}_{1}-\psi_{1} \bar{\psi}_{2}\right)\right\}
\end{aligned}
$$

and the total Lagrangian density of the corresponding reduced model is

$$
\begin{aligned}
-\frac{2 \pi}{k} \mathcal{L} & =\partial_{+} \phi \partial_{-} \phi+\partial_{+} \varphi \partial_{-} \varphi+\psi_{1} \partial_{-} \psi_{1}+\psi_{2} \partial_{-} \psi_{2}+\bar{\psi}_{1} \partial_{+} \bar{\psi}_{1}+\bar{\psi}_{2} \partial_{+} \bar{\psi}_{2}-V \\
V & =\mu^{2}\left\langle\Lambda_{+}^{(+1)} B \Lambda_{-}^{(-1)} B^{-1}\right\rangle+\mu\left\langle\psi_{+}^{(+1 / 2)} B \psi_{-}^{(-1 / 2)} B^{-1}\right\rangle .
\end{aligned}
$$

Now comes the interesting part. To compute the supercharges associated to (126) we use the general formula (68) in the gauge $A_{ \pm}=0$ because $\mathcal{K}_{B}^{(0)}=\oslash$, which reduce to (69) in the mKdV hierarchy. Writing the supercharges in the

\footnotetext{
${ }^{33}$ The $A, S$ stands for $A d S_{n}$ and $S^{n}$.
} 
form $Q\left(\delta_{ \pm 1 / 2}\right)=\int_{-\infty}^{+\infty} d x^{1} G\left(\delta_{ \pm 1 / 2}\right)$, we find the densities $G\left(\delta_{ \pm 1 / 2}\right)=-q_{i}^{ \pm} F_{i}^{(\mp 1 / 2)}$ in terms of the following $2+2$ real components 34

$$
\begin{aligned}
& S F q_{1}^{+}=\psi_{1} \partial_{+} \varphi+\psi_{2} \partial_{+} \phi+\mu\left(\bar{\psi}_{1} \cosh \phi \sin \varphi+\bar{\psi}_{2} \sinh \phi \cos \varphi\right), \\
& S F q_{2}^{+}=\psi_{1} \partial_{+} \phi-\psi_{2} \partial_{+} \varphi+\mu\left(\bar{\psi}_{1} \sinh \phi \cos \varphi-\bar{\psi}_{2} \cosh \phi \sin \varphi\right), \\
& S F q_{1}^{-}=-\bar{\psi}_{1} \partial_{-} \varphi+\bar{\psi}_{2} \partial_{-} \phi+\mu\left(\psi_{1} \cosh \phi \sin \varphi-\psi_{2} \sinh \phi \cos \varphi\right), \\
& S F q_{2}^{-}=\bar{\psi}_{1} \partial_{-} \phi+\bar{\psi}_{2} \partial_{-} \varphi-\mu\left(\psi_{1} \sinh \phi \cos \varphi+\psi_{2} \cosh \phi \sin \varphi\right)
\end{aligned}
$$

and this is because we have $\operatorname{dim} \mathcal{K}_{F}^{( \pm 1 / 2)}=2$, as shown in (115), (143), (145). Compare (127) with the superspace result (121).

Inserting $D^{(+1 / 2)}=\epsilon_{i} F_{i}^{(+1 / 2)}, D^{(-1 / 2)}=\bar{\epsilon}_{i} F_{i}^{(-1 / 2)}$ in the supersymmetry variations (94), (95) and using (147) we obtain the $(2,2)$ supersymmetry algebra

$$
\left[\delta_{+1 / 2}, \delta_{+1 / 2}^{\prime}\right]=2\left(\epsilon_{1} \epsilon_{1}^{\prime}+\epsilon_{2} \epsilon_{2}^{\prime}\right) \partial_{+}, \quad\left[\delta_{-1 / 2}, \delta_{-1 / 2}^{\prime}\right]=2\left(\bar{\epsilon}_{1} \bar{\epsilon}_{1}^{\prime}+\bar{\epsilon}_{2} \bar{\epsilon}_{2}^{\prime}\right) \partial_{-}, \quad\left[\delta_{+1 / 2}, \delta_{-1 / 2}\right]=0
$$

in agreement with the result (99), (100) in the absence of gauge group. These supersymmetry transformations $\delta_{ \pm 1 / 2}$ are the same as the ones induced by the supercharges (121). See also [ 6 for similar models constructed from the twisted superalgebras $\operatorname{sl}(2 \mid 1)^{(2)}$ and $\operatorname{psl}(2 \mid 2)^{(2)}$.

\subsubsection{Reduction of the $A d S_{3} \times S^{3}$ superstring and possible $(4,4) 2 \mathrm{D}$ SUSY.}

This model is more complicated because it has gauge symmetries and it is the first non-trivial case in which we want to test our construction, then we will study it in some detail. Although the Pohlmeyer reduced Lagrangian for the $A d S_{3} \times S^{3}$ superstring was already computed in [2, the existence of $2 \mathrm{D}$ world-sheet supersymmetry was conjectured to be of type $N=(2,2)$. Here we provide some evidence that the $2 \mathrm{D}$ supersymmetry is of the extended type $N=(4,4)$ instead of $N=(2,2)$.

From the general discussion we identify $\frac{F}{G}=\frac{P S U(1,1 \mid 2) \times P S U(1,1 \mid 2)}{S U(1,1) \times S U(2)}$ and $\frac{G}{H}=\frac{S U(1,1) \times S U(2)}{U(1) \times U(1)}$, then this model has gauge group $H=U(1) \times U(1)$ as shown in (148). We will concentrate on the vector gauge only, i.e $\epsilon_{L}=\epsilon_{R}=I$ as the axial gauge i.e $\epsilon_{L}=I, \epsilon_{R}=-I$ follows exactly the same lines.

Using the basis (152), we parametrize the physical fields as follows

$$
B=\operatorname{diag}\left(B_{A}, B_{S}\right), \quad \psi^{(-1 / 2)}=\psi_{i} G_{i}^{(-1 / 2)}, \quad \psi^{(+1 / 2)}=\bar{\psi}_{i} G_{i}^{(+1 / 2)},
$$

where $i=1, \ldots, 4$ and $B_{A} \in S U(1,1), B_{S} \in S U(2)$. The group elements $B_{A}$ and $B_{S}$ are given by

$$
\begin{aligned}
& B_{A}=\exp \left(\frac{1}{2}(\chi+t) K_{1}^{(0)}\right) \exp \left(\phi M_{1}^{(0)}\right) \exp \left(\frac{1}{2}(\chi-t) K_{1}^{(0)}\right) \in S U(1,1), \\
& B_{S}=\exp \left(\frac{1}{2}\left(\theta+t^{\prime}\right) K_{2}^{(0)}\right) \exp \left(\varphi M_{4}^{(0)}\right) \exp \left(\frac{1}{2}\left(\theta-t^{\prime}\right) K_{2}^{(0)}\right) \in S U(2) .
\end{aligned}
$$

The gauge transformations (53) acting on $B$ are simply the shifts $\left(t, t^{\prime}\right) \rightarrow\left(t+\alpha, t^{\prime}+\beta\right)$ and we fix the gauge by taking $t=t^{\prime}=0$ to get, in terms of $2 \times 2$ block matrices, the coset elements

$$
\begin{aligned}
B_{A}^{\prime} & =\left(\begin{array}{cc}
X_{1}+i X_{2} & X_{3} \\
X_{3} & X_{1}-i X_{2}
\end{array}\right) \in \frac{S U(1,1)}{U(1)}, \\
B_{S}^{\prime} & =\left(\begin{array}{cc}
Y_{1}+i Y_{2} & i Y_{3} \\
i Y_{3} & Y_{1}-i Y_{2}
\end{array}\right) \in \frac{S U(2)}{U(1)}
\end{aligned}
$$

\footnotetext{
${ }^{34} \mathrm{SF}$ means Supersymmetry Flows.
} 
where

$$
\begin{aligned}
& X_{1}=\cos \chi \cosh \phi, \quad X_{2}=\sin \chi \cosh \phi, \quad X_{3}=\sqrt{-1+X_{1}^{2}+X_{2}^{2}}=\sinh \phi \\
& Y_{1}=\cos \theta \cos \varphi, \quad Y_{2}=\sin \theta \cos \varphi, \quad Y_{3}=\sqrt{1-Y_{1}^{2}-Y_{2}^{2}}=\sin \varphi
\end{aligned}
$$

The other necessary ingredient is the constant element $\Lambda$ defined in (150) and the gauge fields $A_{ \pm}=a_{ \pm} K_{1}^{(0)}+b_{ \pm} K_{2}^{(0)}$. Using the gauge field $A_{ \pm}$equations of motion given by (54) we find

$$
a_{ \pm}=\mp \frac{1}{2}\left(\operatorname{coth}^{2} \phi \partial_{ \pm} \chi \pm \frac{1}{\sinh ^{2} \phi} F_{ \pm}\right), \quad b_{ \pm}= \pm \frac{1}{2}\left(\cot ^{2} \varphi \partial_{ \pm} \theta \mp \frac{1}{\sin ^{2} \varphi} F_{ \pm}\right)
$$

where $F_{+}=\left(\psi_{1} \psi_{2}-\psi_{3} \psi_{4}\right), F_{-}=\left(\bar{\psi}_{1} \bar{\psi}_{2}-\bar{\psi}_{3} \bar{\psi}_{4}\right)$ and where we have used (154) and $Q_{ \pm}^{(0)}=F_{ \pm}\left(K_{1}^{(0)}-K_{2}^{(0)}\right)$.

Once the $A_{ \pm}$are solved through their equations of motion (131) we put them back in the gauged fixed Lagrangian obtaining the Pohlmeyer reduced action functional. However, instead of doing this we will integrate them out in the path integral without taking account of the quantum measure Jacobian, which gives the same classical answer. To do this, it is useful to consider the general integration formula

$$
\int D A D \bar{A} \exp \left[-\frac{k}{2 \pi} \int(\bar{A} M A+\bar{A} N+\bar{N} A)\right]=\exp \left[-\frac{k}{2 \pi} \int\left(-\bar{N} M^{-1} N\right)\right],
$$

where $A, \bar{A}, N$ and $\bar{N}$ are vectors and $M$ is an invertible matrix.

The gauge field $A_{ \pm}$independent quantities entering the SSSSG model action (52) are

$$
\begin{aligned}
\left\langle B^{-1} \partial_{+} B B^{-1} \partial_{-} B\right\rangle & =2\left(\partial_{+} \phi \partial_{-} \phi-\cosh ^{2} \phi \partial_{+} \chi \partial_{-} \chi+\partial_{+} \varphi \partial_{-} \varphi+\cos ^{2} \varphi \partial_{+} \theta \partial_{-} \theta\right) \\
\left\langle\psi_{+}^{(+1 / 2)} \partial_{-} \psi^{(-1 / 2)}+\psi_{-}^{(-1 / 2)} \partial_{+} \psi^{(+1 / 2)}\right\rangle & =2\left(\psi_{i} \partial_{-} \psi_{i}+\bar{\psi}_{i} \partial_{+} \bar{\psi}_{i}\right) \\
\mu^{2}\left\langle\Lambda_{+}^{(+1)} B \Lambda_{-}^{(-1)} B^{-1}\right\rangle & =-\frac{1}{2} \mu^{2}(\cos 2 \varphi-\cosh 2 \phi) \\
\mu\left\langle\psi_{+}^{(+1 / 2)} B \psi_{-}^{(-1 / 2)} B^{-1}\right\rangle & =2 \mu\left\{\begin{array}{c}
\sin \varphi \sinh \phi\left(\psi_{1} \bar{\psi}_{3}-\psi_{3} \bar{\psi}_{1}+\psi_{2} \bar{\psi}_{4}-\psi_{4} \bar{\psi}_{2}\right)+ \\
+\cos \varphi \cosh \phi\left[\begin{array}{c}
\cos (\theta+\chi)\left(\psi_{1} \bar{\psi}_{2}-\psi_{2} \bar{\psi}_{1}-\psi_{3} \bar{\psi}_{4}+\psi_{4} \bar{\psi}_{3}\right)+ \\
+\sin (\theta+\chi)\left(\psi_{1} \bar{\psi}_{1}+\psi_{2} \bar{\psi}_{2}+\psi_{3} \bar{\psi}_{3}+\psi_{4} \bar{\psi}_{4}\right)
\end{array}\right]
\end{array}\right\}
\end{aligned}
$$

where we have used (154) in the first line and (155) in the last line.

The gauge field $A_{ \pm}$dependent part of the action is

$$
\begin{aligned}
I^{V} & =\left\langle-A_{-}\left(\partial_{+} B B^{-1}+Q_{+}^{(0)}\right)+A_{+}\left(B^{-1} \partial_{-} B+Q_{-}^{(0)}\right)-A_{-} B A_{+} B^{-1}+A_{+} A_{-}\right\rangle \\
& =a_{+} M_{A} a_{-}+a_{+} N_{A}+\bar{N}_{A} a_{-}+b_{+} M_{S} b_{-}+b_{+} N_{S}+\bar{N}_{S} b_{-},
\end{aligned}
$$

where $M_{A}=4 \sinh ^{2} \phi, N_{A}=-2 X_{-}, \bar{N}_{A}=2 X_{+}, M_{S}=4 \sin ^{2} \varphi, N_{S}=2 Y_{-}, \bar{N}_{S}=-2 Y_{+}$and

$$
\begin{aligned}
X_{+} & =\partial_{+} \chi \cosh ^{2} \phi+F_{+}, & X_{-} & =\partial_{-} \chi \cosh ^{2} \phi-F_{-}, \\
Y_{+} & =\partial_{+} \theta \cos ^{2} \varphi-F_{+}, & Y_{-} & =\partial_{+} \theta \cos ^{2} \varphi+F_{-} .
\end{aligned}
$$

Before performing the full $a_{ \pm}, b_{ \pm}$integration, by using (132), we will consider first the following two consistent truncations of the total model which are defined by

$$
\begin{aligned}
& I \quad: \quad B_{S}=I d, \psi_{1}=\psi_{2}=\bar{\psi}_{1}=\bar{\psi}_{2}=0 \text { and } b_{ \pm}=0, \\
& I I \quad: \quad B_{A}=I d, \psi_{3}=\psi_{4}=\bar{\psi}_{3}=\bar{\psi}_{4}=0 \text { and } a_{ \pm}=0 \text {. }
\end{aligned}
$$


One comment is in order. The gauges $b_{ \pm}=0$ or $a_{ \pm}=0$ are only valid on-shell so they does not make any sense at the Lagrangian level. What we are doing is localizing in (48) only one part of the gauge symmetry (50) while keeping the other part intact, i.e global. This is equivalent to the vanishing of some components of $A_{ \pm}$in the action (52). Then, in the limit $I$ we get from (134) and (132)

$$
I_{I}^{V}=-\bar{N}_{A} M_{A}^{-1} N_{A}=\frac{X_{+} X_{-}}{\sinh ^{2} \phi} .
$$

Putting all together with (133) in this particular limit, we get the Lagrangian density

$$
\begin{aligned}
-\frac{2 \pi}{k} \mathcal{L}_{I}= & \partial_{+} \phi \partial_{-} \phi+\operatorname{coth}^{2} \phi \partial_{+} \chi \partial_{-} \chi+\psi_{3} \partial_{-} \psi_{3}+\psi_{4} \partial_{-} \psi_{4}+\bar{\psi}_{3} \partial_{+} \bar{\psi}_{3}+\bar{\psi}_{3} \partial_{+} \bar{\psi}_{4}- \\
& -\operatorname{coth}^{2} \phi\left[\partial_{-} \chi \psi_{3} \psi_{4}-\partial_{+} \chi \bar{\psi}_{3} \bar{\psi}_{4}\right]-\frac{1}{\sinh ^{2} \phi} \psi_{3} \psi_{4} \bar{\psi}_{3} \bar{\psi}_{4}- \\
& -\mu^{2} \sinh ^{2} \phi-2 \mu \cosh \phi\left[\cos \chi\left(-\psi_{3} \bar{\psi}_{4}+\psi_{4} \bar{\psi}_{3}\right)+\sin \chi\left(\psi_{3} \bar{\psi}_{3}+\psi_{4} \bar{\psi}_{4}\right)\right]
\end{aligned}
$$

which should be compared with (124).

After the $a_{ \pm}$gauge field integration there a residual $U(1)$ global symmetry which we now proceed to identify. The lagrangian (135) is separately invariant under the following global transformations

$$
\tilde{\chi}=\chi+\alpha_{1}, \quad \tilde{\lambda}_{ \pm}=e^{ \pm i \beta_{1}} \lambda_{ \pm},
$$

where $\alpha_{1}=2 \pi n, \beta_{1} \in \mathbb{R}, \lambda_{+}=\psi_{3}+i \psi_{4}$ and $\lambda_{-}=\bar{\psi}_{3}-i \bar{\psi}_{4}$. The Noether procedure gives the corresponding charges

$$
Q_{\chi}=\int_{-\infty}^{+\infty} d x^{1} \operatorname{coth}^{2} \phi\left\{\left(\partial_{+} \chi+\partial_{-} \chi\right)-\left(\psi_{3} \psi_{4}-\bar{\psi}_{3} \bar{\psi}_{4}\right)\right\}, \quad Q_{\lambda}=\int_{-\infty}^{+\infty} d x^{1}\left(\psi_{3} \psi_{4}-\bar{\psi}_{3} \bar{\psi}_{4}\right),
$$

where $Q_{\chi}$ is associated to the isometry of the metric and $Q_{\lambda}$ to the fermion electric charge.

Let us extract the information encoded in the conservation law given by the zero grade equations of the DrinfeldSokolov procedure in this Vector gauge. From (70) we have an Abelian charge

$$
Q_{U(1)}=\frac{1}{2} \int_{-\infty}^{+\infty} d x^{1}\left(a_{+}-a_{-}\right)
$$

Now, taking the gauge field components $a_{ \pm}$defined by (131) in this particular limit and using $Q_{\chi}, Q_{\lambda}$, we find that $Q_{U(1)}=-\left(Q_{\chi}+Q_{\lambda}\right)$. Then, the grade zero equations encode the conservations laws associated to the global symmetries of the reduced gauge fixed action.

To compute the supercharges associated to (135), i.e the grade $\pm 1 / 2$ DS equations, we use the general formula (68) in the gauge (131) and neglect the Wilson line conjugation. Writing $Q\left(\delta_{ \pm 1 / 2}\right)=\int_{-\infty}^{+\infty} d x^{1} G\left(\delta_{ \pm 1 / 2}\right)$ and using (156), (157) we can write the fermionic current densities $G\left(\delta_{ \pm 1 / 2}\right)=q_{i}^{ \pm} F_{i}^{(\mp 1 / 2)}$ in terms of the following $2+2$ real components

$$
\begin{aligned}
& S F q_{1}^{+}=\frac{1}{X_{3}}\left(-\psi_{3} \partial_{+} X_{1}+\psi_{4} \partial_{+} X_{2}\right)-\mu \bar{\psi}_{4} X_{3}, \\
& S F q_{2}^{+}=\frac{1}{X_{3}}\left(-\psi_{3} \partial_{+} X_{2}-\psi_{4} \partial_{+} X_{1}\right)+\mu \bar{\psi}_{3} X_{3}, \\
& S F q_{1}^{-}=\frac{1}{X_{3}}\left(-\bar{\psi}_{3} \partial_{-} X_{1}-\bar{\psi}_{4} \partial_{-} X_{2}\right)-\mu \psi_{4} X_{3}, \\
& S F q_{2}^{-}=\frac{1}{X_{3}}\left(\bar{\psi}_{3} \partial_{-} X_{2}-\bar{\psi}_{4} \partial_{-} X_{1}\right)+\mu \psi_{3} X_{3} .
\end{aligned}
$$

which have to be compared with the superspace expressions (125). 
Similarly, in the limit $I I$ we get

$$
I_{I I}^{V}=-\bar{N}_{S} M_{S}^{-1} N_{S}=\frac{Y_{+} Y_{-}}{\sin ^{2} \varphi}
$$

and the Lagrangian density

$$
\begin{aligned}
-\frac{2 \pi}{k} \mathcal{L}_{I I}= & \partial_{+} \varphi \partial_{-} \varphi+\cot ^{2} \varphi \partial_{+} \theta \partial_{-} \theta+\psi_{1} \partial_{-} \psi_{1}+\psi_{2} \partial_{-} \psi_{2}+\bar{\psi}_{1} \partial_{+} \bar{\psi}_{1}+\bar{\psi}_{2} \partial_{+} \bar{\psi}_{2}- \\
& -\cot ^{2} \varphi\left[\partial_{-} \theta \psi_{1} \psi_{2}-\partial_{+} \theta \bar{\psi}_{1} \bar{\psi}_{2}\right]-\frac{1}{\sin ^{2} \varphi} \psi_{1} \psi_{2} \bar{\psi}_{1} \bar{\psi}_{2}- \\
& -\mu^{2} \sin ^{2} \varphi-2 \mu \cos \varphi\left[\cos \theta\left(\psi_{1} \bar{\psi}_{2}-\psi_{2} \bar{\psi}_{1}\right)+\sin \theta\left(\psi_{1} \bar{\psi}_{1}+\psi_{2} \bar{\psi}_{2}\right)\right]
\end{aligned}
$$

which should be compared with (122). Similarly, the $U(1)$ global symmetry of (138) is

$$
\widetilde{\theta}=\theta+\alpha_{2}, \quad \widetilde{\rho}_{ \pm}=e^{ \pm i \beta_{2}} \rho_{ \pm},
$$

where $\alpha_{2}=2 \pi n, \beta_{2} \in \mathbb{R}, \rho_{+}=\psi_{1}+i \psi_{2}$ and $\rho_{-}=\bar{\psi}_{1}-i \bar{\psi}_{2}$. The Noether charges are

$$
Q_{\theta}=\int_{-\infty}^{+\infty} d x^{1} \cot ^{2} \varphi\left\{\left(\partial_{+} \theta+\partial_{-} \theta\right)-\left(\psi_{1} \psi_{2}-\bar{\psi}_{1} \bar{\psi}_{2}\right)\right\}, \quad Q_{\rho}=\int_{-\infty}^{+\infty} d x^{1}\left(\psi_{1} \psi_{2}-\bar{\psi}_{1} \bar{\psi}_{2}\right)
$$

and as above, we have from (70) an Abelian charge given by

$$
Q_{U(1)}=\frac{1}{2} \int_{-\infty}^{+\infty} d x^{1}\left(b_{+}-b_{-}\right) .
$$

Taking $b_{ \pm}$defined by (131) in this particular limit and $Q_{\theta}, Q_{\rho}$, we obtain the relation $Q_{U(1)}=+\left(Q_{\theta}-Q_{\rho}\right)$.

The superdensities can be written again as $G\left(\delta_{ \pm 1 / 2}\right)=q_{i}^{ \pm} F_{i}^{(\mp 1 / 2)}$ in terms of $2+2$ real components

$$
\begin{aligned}
S F q_{1}^{+} & =\frac{1}{Y_{3}}\left(-\psi_{1} \partial_{+} Y_{2}+\psi_{2} \partial_{+} Y_{1}\right)-\mu \bar{\psi}_{1} Y_{3}, \\
S F q_{2}^{+} & =\frac{1}{Y_{3}}\left(-\psi_{1} \partial_{+} Y_{1}-\psi_{2} \partial_{+} Y_{2}\right)-\mu \bar{\psi}_{2} Y_{3}, \\
S F & =\frac{1}{Y_{3}}\left(\bar{\psi}_{1} \partial_{-} Y_{2}+\bar{\psi}_{2} \partial_{-} Y_{1}\right)-\mu \psi_{1} Y_{3}, \\
S F q_{2}^{-} & =\frac{1}{Y_{3}}\left(-\bar{\psi}_{1} \partial_{-} Y_{1}+\bar{\psi}_{2} \partial_{-} Y_{2}\right)-\mu \psi_{2} Y_{3},
\end{aligned}
$$

which should be compared with the superspace expressions (123).

Now we perform the total $a_{ \pm}, b_{ \pm}$integration. This gives for (134) that

$$
I^{V}=\frac{X_{+} X_{-}}{\sinh ^{2} \phi}+\frac{Y_{+} Y_{-}}{\sin ^{2} \varphi}
$$

Putting all together we get the full Pohlmeyer reduced $A d S_{3} \times S^{3}$ superstring sigma model action (see [2])

$$
\begin{aligned}
-\frac{2 \pi}{k} \mathcal{L}= & \partial_{+} \phi \partial_{-} \phi+\operatorname{coth}^{2} \phi \partial_{+} \chi \partial_{-} \chi+\partial_{+} \varphi \partial_{-} \varphi+\cot ^{2} \varphi \partial_{+} \theta \partial_{-} \theta+\left(\psi_{i} \partial_{-} \psi_{i}+\bar{\psi}_{i} \partial_{+} \bar{\psi}_{i}\right)- \\
& -\operatorname{coth}^{2} \phi\left[\partial_{+} \chi F_{-}-\partial_{-} \chi F_{+}\right]+\cot ^{2} \varphi\left[\partial_{+} \theta F_{-}-\partial_{-} \theta F_{+}\right]-F_{+} F_{-}\left(\frac{1}{\sinh ^{2} \phi}+\frac{1}{\sin ^{2} \varphi}\right)-V_{\text {vec }} \\
V_{\text {vec }}= & \mu^{2}\left\langle E_{+}^{(+1)} B E_{-}^{(-1)} B^{-1}\right\rangle+\mu\left\langle\psi_{+}^{(+1 / 2)} B \psi_{-}^{(-1 / 2)} B^{-1}\right\rangle,
\end{aligned}
$$

where $i=1, \ldots, 4$ and the quantities in $V_{\text {vec }}$ are given in (133). Note that in the final form (141), there is no way to take any field limit leading to (135) and (138) and we see how the two sub-models get coupled in a non-trivial way. 
The Lagrangian (141) is separately invariant under (136), (139) and it is also invariant under

$$
\tilde{\chi}=\chi+\alpha_{1}, \quad \tilde{\theta}=\theta+\alpha_{2}, \quad \tilde{\lambda}_{ \pm}=e^{ \pm i \beta} \lambda_{ \pm}, \quad \widetilde{\rho}_{ \pm}=e^{\mp i \beta} \rho_{ \pm},
$$

where we have set $\beta_{2}=-\beta_{1}, \beta_{1}=\beta$. The Noether charges are

$$
\begin{aligned}
Q_{\chi} & =\int_{-\infty}^{+\infty} d x^{1} \operatorname{coth}^{2} \phi\left\{\left(\partial_{+} \chi+\partial_{-} \chi\right)+\left(F_{+}-F_{-}\right)\right\} \\
Q_{\theta} & =\int_{-\infty}^{+\infty} d x^{1} \cot ^{2} \varphi\left\{\left(\partial_{+} \theta+\partial_{-} \theta\right)-\left(F_{+}-F_{-}\right)\right\} \\
Q_{\rho, \lambda} & =\int_{-\infty}^{+\infty} d x^{1}\left(F_{+}-F_{-}\right) .
\end{aligned}
$$

From (70) we have the residual $U(1) \times U(1)$ global symmetry with conserved charges

$$
Q_{a}=\frac{1}{2} \int_{-\infty}^{+\infty}\left(a_{+}-a_{-}\right), \quad Q_{b}=\frac{1}{2} \int_{-\infty}^{+\infty}\left(b_{+}-b_{-}\right)
$$

and with $a_{ \pm}, b_{ \pm}$defined by (131), we find the relations

$$
Q_{a}=-\left(Q_{\chi}-Q_{\rho, \lambda}\right), \quad Q_{b}=+\left(Q_{\theta}-Q_{\rho, \lambda}\right)
$$

The eight fermionic densities, because we have $\operatorname{dim} \mathcal{K}_{F}^{( \pm 1 / 2)}=4$ as can be seen from (115), (149), (151), associated to the reduced model are computed from 35 (68). They can be written as $G\left(\delta_{ \pm 1 / 2}\right)=q_{i}^{ \pm} F_{i}^{(\mp 1 / 2)}$, where

$$
\begin{aligned}
S F q_{1}^{+}= & \frac{1}{X_{3}}\left[-\psi_{3}\left(\partial_{+} X_{1}-X_{2} F_{+}\right)+\psi_{4}\left(\partial_{+} X_{2}+X_{1} F_{+}\right)\right]+\frac{1}{Y_{3}}\left[-\psi_{1}\left(\partial_{+} Y_{2}-Y_{1} F_{+}\right)+\psi_{2}\left(\partial_{+} Y_{1}+Y_{2} F_{+}\right)\right]+ \\
& +\mu\left(-\bar{\psi}_{1} X_{1} Y_{3}+\bar{\psi}_{2} X_{2} Y_{3}+\bar{\psi}_{3} X_{3} Y_{2}-\bar{\psi}_{4} X_{3} Y_{1}\right) \\
S F q_{2}^{+}= & \frac{1}{X_{3}}\left[-\psi_{3}\left(\partial_{+} X_{2}+X_{1} F_{+}\right)-\psi_{4}\left(\partial_{+} X_{1}-X_{2} F_{+}\right)\right]+\frac{1}{Y_{3}}\left[-\psi_{1}\left(\partial_{+} Y_{1}+Y_{2} F_{+}\right)-\psi_{2}\left(\partial_{+} Y_{2}-Y_{1} F_{+}\right)\right]+ \\
& +\mu\left(-\bar{\psi}_{1} X_{2} Y_{3}-\bar{\psi}_{2} X_{1} Y_{3}+\bar{\psi}_{3} X_{3} Y_{1}+\bar{\psi}_{4} X_{3} Y_{2}\right), \\
S F q_{3}^{+}= & \frac{1}{X_{3}}\left[-\psi_{1}\left(\partial_{+} X_{1}-X_{2} F_{+}\right)-\psi_{2}\left(\partial_{+} X_{2}+X_{1} F_{+}\right)\right]+\frac{1}{Y_{3}}\left[\psi_{3}\left(\partial_{+} Y_{2}-Y_{1} F_{+}\right)+\psi_{4}\left(\partial_{+} Y_{1}+Y_{2} F_{+}\right)\right]+ \\
& +\mu\left(\bar{\psi}_{1} X_{3} Y_{2}+\bar{\psi}_{2} X_{3} Y_{1}+\bar{\psi}_{3} X_{1} Y_{3}+\bar{\psi}_{4} X_{2} Y_{3}\right), \\
S F q_{4}^{+}= & \frac{1}{X_{3}}\left[\psi_{1}\left(\partial_{+} X_{2}+X_{1} F_{+}\right)-\psi_{2}\left(\partial_{+} X_{1}-X_{2} F_{+}\right)\right]+\frac{1}{Y_{3}}\left[-\psi_{3}\left(\partial_{+} Y_{1}+Y_{2} F_{+}\right)+\psi_{4}\left(\partial_{+} Y_{2}-Y_{1} F_{+}\right)\right]+ \\
& +\mu\left(-\bar{\psi}_{1} X_{3} Y_{1}+\bar{\psi}_{2} X_{3} Y_{2}-\bar{\psi}_{3} X_{2} Y_{3}+\bar{\psi}_{4} X_{1} Y_{3}\right)
\end{aligned}
$$

and

$$
\begin{aligned}
S F q_{1}^{-}= & \frac{1}{X_{3}}\left[-\bar{\psi}_{3}\left(\partial_{-} X_{1}+X_{2} F_{-}\right)-\bar{\psi}_{4}\left(\partial_{-} X_{2}-X_{1} F_{-}\right)\right]+\frac{1}{Y_{3}}\left[\bar{\psi}_{1}\left(\partial_{-} Y_{2}+Y_{1} F_{-}\right)+\bar{\psi}_{2}\left(\partial_{-} Y_{1}-Y_{2} F_{-}\right)\right]+ \\
& +\mu\left(-\psi_{1} X_{1} Y_{3}-\psi_{2} X_{2} Y_{3}-\psi_{3} X_{3} Y_{2}-\psi_{4} X_{3} Y_{1}\right), \\
S F q_{2}^{-}= & \frac{1}{X_{3}}\left[\bar{\psi}_{3}\left(\partial_{-} X_{2}-X_{1} F_{-}\right)-\bar{\psi}_{4}\left(\partial_{-} X_{1}+X_{2} F_{-}\right)\right]+\frac{1}{Y_{3}}\left[-\bar{\psi}_{1}\left(\partial_{-} Y_{1}-Y_{2} F_{-}\right)+\bar{\psi}_{2}\left(\partial_{-} Y_{2}+Y_{1} F_{-}\right)\right]+ \\
& +\mu\left(\psi_{1} X_{2} Y_{3}-\psi_{2} X_{1} Y_{3}+\psi_{3} X_{3} Y_{1}-\psi_{4} X_{3} Y_{2}\right), \\
S F q_{3}^{-}= & \frac{1}{X_{3}}\left[-\bar{\psi}_{1}\left(\partial_{-} X_{1}+X_{2} F_{-}\right)+\bar{\psi}_{2}\left(\partial_{-} X_{2}-X_{1} F_{-}\right)\right]+\frac{1}{Y_{3}}\left[-\bar{\psi}_{3}\left(\partial_{-} Y_{2}+Y_{1} F_{-}\right)+\bar{\psi}_{4}\left(\partial_{-} Y_{1}-Y_{2} F_{-}\right)\right]+ \\
& +\mu\left(-\psi_{1} X_{3} Y_{2}+\psi_{2} X_{3} Y_{1}+\psi_{3} X_{1} Y_{3}-\psi_{4} X_{2} Y_{3}\right), \\
S F q_{4}^{-}= & \frac{1}{X_{3}}\left[-\bar{\psi}_{1}\left(\partial_{-} X_{2}-X_{1} F_{-}\right)-\bar{\psi}_{2}\left(\partial_{-} X_{1}+X_{2} F_{-}\right)\right]+\frac{1}{Y_{3}}\left[-\bar{\psi}_{3}\left(\partial_{-} Y_{1}-Y_{2} F_{-}\right)-\bar{\psi}_{4}\left(\partial_{-} Y_{2}+Y_{1} F_{-}\right)\right]+ \\
& +\mu\left(-\psi_{1} X_{3} Y_{1}-\psi_{2} X_{3} Y_{2}+\psi_{3} X_{2} Y_{3}+\psi_{4} X_{1} Y_{3}\right),
\end{aligned}
$$

\footnotetext{
${ }^{35}$ Recall that we are not writing the Wilson lines.
} 
are mixings of the two sub-sets of charges (137) and (140). From the kernel superalgebra (153) we expect the reduced model to have an extended $(4,4) 2 \mathrm{D}$ supersymmetry algebra with supercharges transforming under the $U(1) \times U(1)$ gauge group. Of course, this is an on-shell symmetry as shown above. We leave for the future the study of the field variations associated to the non-local charges (68) and the possible invariance of the gauge fixed action functional (52) under them.

As we have shown there are two corners in field space in which we have two submodels (loosely) related to the $(2,2)$ supersymmetric models (135) and (138). Possibly, these models are not related to the $A d S_{3} \times S^{3}$ superstring because the reduction procedure requires the whole gauge symmetry group $H$ to be local and not only a part of it. However, it would be interesting to see if it is possible to obtain these submodels as particular limits of the radius of the coset $F / G$, i.e the constant $\kappa$ in the GS action (104). In any case, it seems to be that in the reduction process these two submodels are entangled in the form (141) with a (possibly non-local) extended $(4,4)$ supersymmetry. This is argument is based solely in the number of non-local supercharges constructed above and, of course, a deeper study have to be done 36 .

\section{Concluding remarks.}

We have provided substantial evidence that the conjectured existence of the world-sheet supersymmetry of the Pohlmeyer reduced models is of the extended type and generated by the kernel loop superalgebra $\mathcal{K}$ constructed out of the subalgebra $\mathfrak{f}^{\perp} \subset \mathfrak{f}$. Of course, on-shell $\mathcal{K}$ is a true algebra of symmetries which leave invariant the equations of motion and one of the most difficult issues yet to be solved, is to show the invariance of the gauge fixed SSSSG model action functional under an appropriate set of residual symmetry variations $\delta_{\mathcal{K}}$. For the moment, the supersymmetries just constructed are non manifest at the lagrangian level and the problem of making them manifest as well as the full Poisson form of the supersymmetry algebra in terms of the monodromy matrix will be addressed in the near future 10. If the action is SUSY invariant, perhaps we can try to use localization techniques to handle the partition function and to study its properties, at least in the conformal $A d S_{5} \times S^{5}$ superstring in which the Pohlmeyer reduction have a chance to survive the quantization 25. Another important problem to be studied is the construction and quantization of soliton solutions involving the fermionic fields for the set of semi-symmetric superspaces $F / G$ associated to several Lie superalgebras $\mathfrak{f}$ admiting a $\mathbb{Z}_{4}$ decomposition. Fortunately, this can be done by extending to the supersymmetric case, the results recently presented in [17] for the bosonic symmetric-space sine-Gordon models.

Acknowledgements. I would like to thank the IGFAE (Santiago de Compostela, Spain) for the kind hospitality extended while this work was in final preparation. I want to thank Alexis R. Aguirre, José F. Gomes, Timothy J. Hollowood, J. Luis Miramontes, Arkady Tseytlin and Abraham H. Zimerman for many useful comments, advices, suggestions, discussions and correspondence. Special thanks to J. Luis Miramontes for making available a preliminary version of the forthcoming paper [9]. This research is supported by a CNPq junior postdoctoral grant.

\section{Appendix A: The superalgebra $p s u(1,1 \mid 2)$}

Consider the following distinguished Dynkin diagram of the superalgebra $\operatorname{sl}(2 \mid 2)_{\mathbb{C}}$

$$
\underset{\alpha_{1}}{\bigcirc}-\underset{\alpha_{2}}{\otimes}-\underset{\alpha_{3}}{\bigcirc},
$$

\footnotetext{
${ }^{36}$ I want to thank Arkady Tseytlin for pointing me several subtleties related to the hyperkahlerianity and reducibility of the target manifold in relation to $(4,4)$ SUSY.
} 
where $\alpha_{1}, \alpha_{3}$ are the bosonic simple roots and $\alpha_{2}$ is the fermionic simple root. Introduce the step operators $E_{ \pm \alpha_{1}}$, $E_{ \pm \alpha_{2}}, E_{ \pm \alpha_{3}}, E_{ \pm \alpha_{1} \pm \alpha_{2}}, E_{ \pm \alpha_{2} \pm \alpha_{3}}, E_{ \pm \alpha_{1} \pm \alpha_{2} \pm \alpha_{3}}$, the Cartan elements $h_{1}, h_{2}, h_{3}, I$ and work in a $4 \times 4$ supermatrix representation. Then, by introducing the matrices $\left(E_{a b}\right)_{i j}=\delta_{a i} \delta_{b j}, a, b, i, j=1,2,3,4$, we have

$$
\begin{aligned}
E_{\alpha_{1}} & =E_{12}, \quad E_{\alpha_{2}}=E_{23}, \quad E_{\alpha_{3}}=E_{34}, \quad E_{\alpha_{1}+\alpha_{2}}=E_{13}, \quad E_{\alpha_{2}+\alpha_{3}}=E_{24}, \quad E_{\alpha_{1}+\alpha_{2}+\alpha_{3}}=E_{14}, \\
h_{1} & =E_{11}-E_{22}, \quad h_{2}=E_{22}+E_{33}, \quad h_{3}=E_{33}-E_{44},
\end{aligned}
$$

where the matrices corresponding to the negative roots are represented by the transpose of the matrix corresponding to the positive root, e.g $E_{-\alpha_{1}}=\left(E_{\alpha_{1}}\right)^{t}$. We introduce these matrices in order to write the base of the $s u(1,1 \mid 2)$ superalgebra in terms of them.

\section{1 $\mathbb{Z}_{4}$ grading and $\mathfrak{f}^{\perp}, \mathfrak{f}^{\|}$decomposition of $\operatorname{psu}(1,1 \mid 2)$.}

An element $M \subset \operatorname{sl}(2 \mid 2)_{\mathbb{C}}$ can be represented by a $4 \times 4$ supermatrix

$$
M=\left(\begin{array}{cc}
A & X \\
Y & B
\end{array}\right)
$$

where $A, B$ are (even) complex $2 \times 2$ matrices and $X, Y$ are (odd) complex $2 \times 2$ matrices. Introduce the following $4 \times 4$ matrices

$$
\Sigma=\left(\begin{array}{cc}
\sigma & 0 \\
0 & I
\end{array}\right), \quad K=\left(\begin{array}{cc}
\sigma & 0 \\
0 & \sigma
\end{array}\right), \quad \sigma=\left(\begin{array}{cc}
1 & 0 \\
0 & -1
\end{array}\right)
$$

and recall the definition of super-transposition and super-Hermitian conjugation

$$
M^{s t}=\left(\begin{array}{cc}
A^{t} & -Y^{t} \\
X^{t} & B^{t}
\end{array}\right), \quad M^{\dagger}=\left(\begin{array}{cc}
A^{\dagger} & -i Y^{\dagger} \\
-i X^{\dagger} & B^{\dagger}
\end{array}\right) .
$$

The $\mathfrak{f}=\operatorname{psu}(1,1 \mid 2)$ superalgebra is a real form of $\operatorname{psl}(2 \mid 2)_{\mathbb{C}}$ and can be represented by $4 \times 4$ supermatrices modulo the identity matrix. It is defined by $M^{*}=-M$ where $M^{*}=\Sigma M \Sigma$. The $\mathbb{Z}_{4}$ decomposition of $\mathfrak{f}$ is implemented by the action of the following automorphism $M^{\Omega}=-K M^{\text {st }} K$, which allows the splitting of $\mathfrak{f}$ as a direct sum of subspaces $\mathfrak{f}=\mathfrak{f}_{0} \oplus \mathfrak{f}_{1} \oplus \mathfrak{f}_{2} \oplus \mathfrak{f}_{3}$ where each $\mathfrak{f}_{j}$ is the eigen-space of $\Omega$ with eigenvalue $j$ i.e for $M \in \mathfrak{f}_{j}$ we have $M^{\Omega}=i^{j} M$. We also

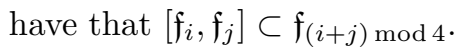

Imposing $M^{*}=-M$ we get $A^{\dagger}=-\sigma A \sigma, B^{\dagger}=-B$ and $Y^{\dagger}=-i \sigma X$ defining respectively the $s u(1,1)$ and $s u(2)$ Lie algebras and reducing the number of odd elements. Using $M^{\Omega}=i^{j} M$ we find that $\mathfrak{f}_{j}$ is formed by the supermatrices obeying

$$
\begin{array}{lll}
\mathfrak{f}_{0}: & A_{0}=-\sigma A_{0}^{t} \sigma, \quad B_{0}=-\sigma B_{0}^{t} \sigma, & \mathfrak{f}_{2}: A_{2}=\sigma A_{2}^{t} \sigma, \quad B_{2}=\sigma B_{2}^{t} \sigma, \\
\mathfrak{f}_{1}: Y=i \sigma X^{t} \sigma, & \mathfrak{f}_{3}: Y=-i \sigma X^{t} \sigma .
\end{array}
$$

Written in terms of step operators we get

$$
\begin{aligned}
& \mathfrak{f}_{0}:\left\{\begin{array}{c}
f_{01}=\left(E_{\alpha_{1}}+E_{-\alpha_{1}}\right) \\
f_{02}=i\left(E_{\alpha_{3}}+E_{-\alpha_{3}}\right)
\end{array}\right\}, \quad \mathfrak{f}_{2}:\left\{\begin{array}{c}
f_{21}=i h_{1} \\
f_{22}=i\left(E_{\alpha_{1}}-E_{-\alpha_{1}}\right) \\
f_{23}=i h_{3} \\
f_{24}=\left(E_{\alpha_{3}}-E_{-\alpha_{3}}\right)
\end{array}\right\}, \\
& \mathfrak{f}_{1}:\left\{\begin{array}{c}
f_{11}=\left(E_{\alpha_{1}+\alpha_{2}}+i E_{-\alpha_{1}-\alpha_{2}}\right) \\
f_{12}=\left(i E_{\alpha_{1}+\alpha_{2}+\alpha_{3}}+E_{-\alpha_{1}-\alpha_{2}-\alpha_{3}}\right) \\
f_{13}=\left(E_{\alpha_{2}}-i E_{-\alpha_{2}}\right) \\
f_{14}=\left(i E_{\alpha_{2}+\alpha_{3}}-E_{-\alpha_{2}-\alpha_{3}}\right)
\end{array}\right\}, \mathfrak{f}_{3}:\left\{\begin{array}{c}
f_{31}=\left(i E_{\alpha_{1}+\alpha_{2}}+E_{-\alpha_{1}-\alpha_{2}}\right) \\
f_{32}=\left(E_{\alpha_{1}+\alpha_{2}+\alpha_{3}}+i E_{-\alpha_{1}-\alpha_{2}-\alpha_{3}}\right) \\
f_{33}=\left(i E_{\alpha_{2}}-E_{-\alpha_{2}}\right) \\
f_{34}=\left(E_{\alpha_{2}+\alpha_{3}}-i E_{-\alpha_{2}-\alpha_{3}}\right)
\end{array}\right\},
\end{aligned}
$$


where in the notation $f_{i a}$ the index $i=0, \ldots, 4$ stands for the $\mathbb{Z}_{4}$ eigenvalue $i$ of the subspace $\mathfrak{f}_{i}$ and the index $a=1, \ldots, \operatorname{dim} \mathfrak{f}_{i}$ is just a basis label.

By defining the semisimple element $\Lambda=\frac{1}{2}\left(f_{21}+f_{23}\right) \in \mathfrak{a}_{2}$, we obtain the decomposition of the superalgebra in the $\mathbb{Z}_{2}$ form $\mathfrak{f}=\mathfrak{f}^{\perp} \oplus \mathfrak{f}^{\|}$, where $\mathfrak{f}^{\perp}=\operatorname{ker}(a d(\Lambda)), \mathfrak{f}^{\|}=\operatorname{Im}(a d(\Lambda))$ and where every subspace $\mathfrak{f}_{i}$ is also decomposed as $\mathfrak{f}_{i}=\mathfrak{f}_{i}^{\perp}+\mathfrak{f}_{i}^{\|}$. We find

$$
\begin{gathered}
\mathfrak{f}_{0}^{\perp}:\{\varnothing\} \rightarrow \text { No gauge flows, } \\
\mathfrak{f}_{0}^{\|}:\left\{\begin{array}{l}
M_{01}=f_{01} \\
M_{02}=f_{02}
\end{array}\right\} \rightarrow \text { } 2 \text { bosonic fields, } \\
\mathfrak{f}_{1}^{\perp}:\left\{\begin{array}{l}
F_{11}=f_{11} \\
F_{12}=f_{14}
\end{array}\right\} \rightarrow \text { 2 SUSY flows, } \\
\mathfrak{f}_{1}^{\|}:\left\{\begin{array}{l}
G_{11}=f_{12} \\
G_{12}=f_{13}
\end{array}\right\} \rightarrow \text { 2 fermionic fields, } \\
\mathfrak{f}_{2}^{\perp}:\left\{\begin{array}{l}
K_{21}=f_{21} \\
K_{22}=f_{23}
\end{array}\right\} \rightarrow \Lambda=\frac{1}{2}\left(K_{21}+K_{22}\right), \\
\mathfrak{f}_{2}^{\|}:\left\{\begin{array}{l}
M_{21}=f_{22} \\
M_{22}=f_{24}
\end{array}\right\} \rightarrow \text { No dynamics, } \\
\mathfrak{f}_{3}^{\perp}:\left\{\begin{array}{l}
F_{31}=f_{31} \\
F_{32}=f_{34}
\end{array}\right\} \rightarrow \text { 2 SUSY flows, } \\
\mathfrak{f}_{3}^{\|}:\left\{\begin{array}{l}
G_{31}=f_{32} \\
G_{32}=f_{33}
\end{array}\right\} \rightarrow \text { 2 fermionic fields. }
\end{gathered}
$$

From the loop superalgebra setting (3), (4), we get the basis elements in $\mathcal{K}=\widehat{\mathfrak{f}}^{\perp}$ and $\mathcal{M}=\widehat{\mathfrak{f}}^{\|}$

$$
\begin{aligned}
& F_{i}^{(-1 / 2)}=z^{-1 / 2} F_{3 i} \in \mathcal{K}_{F}^{(-1 / 2)}, \quad F_{i}^{(+1 / 2)}=z^{+1 / 2} F_{1 i} \in \mathcal{K}_{F}^{(+1 / 2)}, \\
& G_{i}^{(-1 / 2)}=z^{-1 / 2} G_{3 i} \in \mathcal{M}_{F}^{(-1 / 2)}, \quad M_{a}^{(0)}=z^{0} M_{0 a} \in \mathcal{M}_{B}^{(0)}, \quad G_{i}^{(+1 / 2)}=z^{+1 / 2} G_{1 i} \in \mathcal{M}_{F}^{(+1 / 2)} .
\end{aligned}
$$

The kernel algebra $\mathcal{K}$ is

$$
\left\{F_{i}^{( \pm 1 / 2)}, F_{j}^{( \pm 1 / 2)}\right\}= \pm 2 \delta_{i j} \Lambda_{ \pm}^{( \pm 1)}, \quad\left\{F_{i}^{(+1 / 2)}, F_{j}^{(-1 / 2)}\right\}=0
$$

In this case the algebra $\mathcal{K}$ is related through $\delta_{\mathcal{K}}$ to the usual $(2,2)$ supersymmetric extension of the $2 \mathrm{D}$ Poincaré algebra.

\section{$6.2 \mathbb{Z}_{4}$ grading and $\mathfrak{f}^{\perp}, \mathfrak{f}^{\|}$decomposition of $\operatorname{psu}(1,1 \mid 2)^{\times 2}$.}

An element $X \subset \operatorname{sl}(2 \mid 2)_{\mathbb{C}}^{\times 2}$ can be represented by a $8 \times 8$ supermatrix

$$
X=\left(\begin{array}{cc}
M & 0 \\
0 & N
\end{array}\right), \quad M=\left(\begin{array}{cc}
A & X \\
Y & B
\end{array}\right), \quad N=\left(\begin{array}{cc}
C & Z \\
W & D
\end{array}\right),
$$

where $M$ and $N$ are $4 \times 4$ elements of $s l(2 \mid 2)_{\mathbb{C}}$. The $\mathfrak{f}=p s u(1,1 \mid 2)^{\times 2}$ superalgebra is a real form of $p s l(2 \mid 2)_{\mathbb{C}}^{\times 2}$ and can be represented by $8 \times 8$ supermatrices modulo the identity matrix. It is defined by $M^{*}=-M$ and $N=-N^{*}$, with $*$ as above, then the only subtle point relies in the $\mathbb{Z}_{4}$ decomposition [2]. Introduce the following $8 \times 8$ matrices

$$
K=\left(\begin{array}{cc}
0 & k \\
k & 0
\end{array}\right), \quad k=\left(\begin{array}{cc}
I & 0 \\
0 & I
\end{array}\right) .
$$


The $\mathbb{Z}_{4}$ decomposition of $\mathfrak{f}$ is implemented by the action of the following automorphism $M^{\Omega}=-K M^{s t} K$. Using $M^{\Omega}=i^{j} M$ we find that $\mathfrak{f}_{j}$ is formed by the supermatrices $X \in \mathfrak{f}$ obeying

$$
\begin{aligned}
& \mathfrak{f}_{0}:\left(\begin{array}{cccc}
A & 0 & 0 & 0 \\
0 & B & 0 & 0 \\
0 & 0 & -A^{t} & 0 \\
0 & 0 & 0 & -B^{t}
\end{array}\right), \quad \mathfrak{f}_{1}:\left(\begin{array}{cccc}
0 & X & 0 & 0 \\
Y & 0 & 0 & 0 \\
0 & 0 & 0 & -i Y^{t} \\
0 & 0 & i X^{t} & 0
\end{array}\right) \text {, } \\
& \mathfrak{f}_{2}:\left(\begin{array}{cccc}
A & 0 & 0 & 0 \\
0 & B & 0 & 0 \\
0 & 0 & A^{t} & 0 \\
0 & 0 & 0 & B^{t}
\end{array}\right), \quad \mathfrak{f}_{3}:\left(\begin{array}{cccc}
0 & X & 0 & 0 \\
Y & 0 & 0 & 0 \\
0 & 0 & 0 & i Y^{t} \\
0 & 0 & -i X^{t} & 0
\end{array}\right) \text {, }
\end{aligned}
$$

where $Y=i X^{\dagger} \Sigma$ and $\Sigma$ is defined above. It is enough to consider only the first copy of $p s u(1,1 \mid 2)$ inside $\mathfrak{f}$ and we can return to the $4 \times 4$ supermatrix representation of $p s u(1,1 \mid 2)$ used above. Note that now we have $\mathfrak{f}_{0}=\mathfrak{f}_{2}=s u(1,1) \times s u(2)$.

By taking the semisimple element $\Lambda=\frac{1}{2}\left(f_{21}+f_{23}\right) \in \mathfrak{a}_{2}$, we obtain the $\mathbb{Z}_{2}$ decomposition $\mathfrak{f}=\mathfrak{f}^{\perp} \oplus \mathfrak{f}^{\|}$of the superalgebra and every subspace $\mathfrak{f}_{i}$ is decomposed as $\mathfrak{f}_{i}=\mathfrak{f}_{i}^{\perp}+\mathfrak{f}_{i}^{\|}$, with

$$
\begin{aligned}
& \mathfrak{f}_{0}^{\perp}:\left\{\begin{array}{l}
K_{01}=i h_{1} \\
K_{02}=i h_{3}
\end{array}\right\} \rightarrow U(1) \times U(1) \text { gauge flows, } \\
& \mathfrak{f}_{0}^{\|}:\left\{\begin{array}{c}
M_{01}=\left(E_{\alpha_{1}}+E_{-\alpha_{1}}\right) \\
M_{02}=i\left(E_{\alpha_{1}}-E_{-\alpha_{1}}\right) \\
M_{03}=\left(E_{\alpha_{3}}-E_{-\alpha_{3}}\right) \\
M_{04}=i\left(E_{\alpha_{3}}+E_{-\alpha_{3}}\right)
\end{array}\right\} \rightarrow 4 \text { bosonic fields, } \\
& \mathfrak{f}_{1}^{\perp}:\left\{\begin{array}{l}
F_{11}=\left(E_{\alpha_{1}+\alpha_{2}}+i E_{-\alpha_{1}-\alpha_{2}}\right) \\
F_{12}=\left(i E_{\alpha_{1}+\alpha_{2}}+E_{-\alpha_{1}-\alpha_{2}}\right) \\
F_{13}=\left(E_{\alpha_{2}+\alpha_{3}}-i E_{-\alpha_{2}-\alpha_{3}}\right) \\
F_{14}=\left(i E_{\alpha_{2}+\alpha_{3}}-E_{-\alpha_{2}-\alpha_{3}}\right)
\end{array}\right\} \rightarrow \text { 4 SUSY flows, } \\
& f_{1}^{\|}:\left\{\begin{aligned}
G_{11}= & \left(E_{\alpha_{1}+\alpha_{2}+\alpha_{3}}+i E_{-\alpha_{1}-\alpha_{2}-\alpha_{3}}\right) \\
G_{12}= & \left(i E_{\alpha_{1}+\alpha_{2}+\alpha_{3}}+E_{-\alpha_{1}-\alpha_{2}-\alpha_{3}}\right) \\
& G_{13}=\left(E_{\alpha_{2}}-i E_{-\alpha_{2}}\right) \\
& G_{14}=\left(i E_{\alpha_{2}}-E_{-\alpha_{2}}\right)
\end{aligned}\right\} \rightarrow 4 \text { fermionic fields, } \\
& \mathfrak{f}_{2}^{\perp}:\left\{\begin{array}{l}
K_{21}=K_{01} \\
K_{22}=K_{02}
\end{array}\right\} \rightarrow \Lambda=\frac{1}{2}\left(K_{21}+K_{22}\right), \\
& \mathfrak{f}_{2}^{\|}:\left\{\begin{array}{l}
M_{21}=M_{01} \\
M_{22}=M_{02} \\
M_{23}=M_{03} \\
M_{24}=M_{04}
\end{array}\right\} \rightarrow \text { No dynamics } \\
& \mathfrak{f}_{3}^{\perp}:\left\{\begin{array}{l}
F_{31}=F_{11} \\
F_{32}=F_{12} \\
F_{33}=F_{13} \\
F_{34}=F_{14}
\end{array}\right\} \rightarrow 4 \text { SUSY flows }
\end{aligned}
$$




$$
\mathfrak{f}_{3}^{\|}:\left\{\begin{array}{l}
G_{31}=G_{11} \\
G_{32}=G_{12} \\
G_{33}=G_{13} \\
G_{34}=G_{14}
\end{array}\right\} \rightarrow 4 \text { fermionic fields. }
$$

In the loop superalgebra, we have the following basis elements of $\mathcal{K}$ and $\mathcal{M}$

$$
\begin{array}{lll}
F_{i}^{(-1 / 2)}=z^{-1 / 2} F_{3 i} \in \mathcal{K}_{F}^{(-1 / 2)}, & K_{b}^{(0)}=z^{0} K_{0 b} \in \mathcal{K}_{B}^{(0)}, & F_{i}^{(+1 / 2)}=z^{+1 / 2} F_{1 i} \in \mathcal{K}_{F}^{(+1 / 2)}, \\
G_{i}^{(-1 / 2)}=z^{-1 / 2} G_{3 i} \in \mathcal{M}_{F}^{(-1 / 2)}, & M_{a}^{(0)}=z^{0} M_{0 a} \in \mathcal{M}_{B}^{(0)}, & G_{i}^{(+1 / 2)}=z^{+1 / 2} G_{1 i} \in \mathcal{M}_{F}^{(+1 / 2)} .
\end{array}
$$

The kernel algebra $\mathcal{K}$ is

$$
\begin{aligned}
\left\{F_{i}^{( \pm 1 / 2)}, F_{j}^{( \pm 1 / 2)}\right\} & = \pm 2 \delta_{i j} \Lambda_{ \pm}^{( \pm 1)}, \quad\left\{F_{i}^{(+1 / 2)}, F_{j}^{(-1 / 2)}\right\}=2 \delta_{i j} Z^{(0)} \\
{\left[K_{a}^{(0)}, F_{i}^{( \pm 1 / 2)}\right] } & =-(-1)^{a} X_{i j} F_{j}^{( \pm 1 / 2)}, \quad a=1,2
\end{aligned}
$$

where $Z^{(0)}=K_{1}^{(0)}+K_{2}^{(0)}$ commutes with everything in $\mathcal{K}$ and $\left[X_{i j}\right]=\left(\begin{array}{cc}\epsilon & 0 \\ 0 & -\epsilon\end{array}\right)$ with $\epsilon=\left(\begin{array}{cc}0 & 1 \\ -1 & 0\end{array}\right)$. In this case the algebra $\mathcal{K}$ is related through $\delta_{\mathcal{K}}$ to the $N=(4,4)$ supersymmetric extension of the 2D Poincare algebra with a gauge group $U(1) \times U(1)$.

\section{Appendix B: Relevant quantities used in the computations}

Using the definitions of Appendix A, section 6.2 we obtain, respectively, the following currents, traces and conjugations

$$
\begin{aligned}
& \partial_{+} B_{A} B_{A}^{-1}=\left(\partial_{+} \chi \cosh ^{2} \phi\right) K_{1}^{(0)}+\left(\cos \chi \partial_{+} \phi+\partial_{+} \chi \sin \chi \cosh \phi \sinh \phi\right) M_{1}^{(2)}+ \\
& +\left(\sin \chi \partial_{+} \phi-\partial_{+} \chi \cos \chi \cosh \phi \sinh \phi\right) M_{2}^{(0)} \text {, } \\
& \partial_{+} B_{S} B_{S}^{-1}=\left(\partial_{+} \theta \cos ^{2} \varphi\right) K_{2}^{(0)}+\left(-\sin \theta \partial_{+} \varphi+\partial_{+} \theta \cos \theta \cos \varphi \sin \varphi\right) M_{3}^{(2)}+ \\
& +\left(\cos \theta \partial_{+} \varphi+\partial_{+} \theta \sin \theta \cos \varphi \sin \varphi\right) M_{4}^{(0)}, \\
& B_{S}^{-1} \partial_{-} B_{S}=\left(\partial_{-} \chi \cosh ^{2} \phi\right) K_{1}^{(0)}+\left(\cos \chi \partial_{-} \phi+\partial_{-} \chi \sin \chi \cosh \phi \sinh \phi\right) M_{1}^{(0)}+ \\
& +\left(-\sin \chi \partial_{-} \phi+\partial_{-} \chi \cos \chi \cosh \phi \sinh \phi\right) M_{2}^{(0)} \text {, } \\
& B_{S}^{-1} \partial_{-} B_{S}=\left(\partial_{-} \theta \cos ^{2} \varphi\right) K_{2}^{(0)}+\left(\sin \theta \partial_{-} \varphi-\partial_{-} \theta \cos \theta \cos \varphi \sin \varphi\right) M_{3}^{(0)}+ \\
& +\left(\cos \theta \partial_{-} \varphi+\partial_{-} \theta \sin \theta \cos \varphi \sin \varphi\right) M_{4}^{(0)} \text {. } \\
& \left\langle G_{i}^{(+1 / 2)}, B G_{i}^{(-1 / 2)} B^{-1}\right\rangle=2 \cos \varphi \cosh \phi \sin (\theta+\chi), \quad i=1,2,3,4 \\
& \left\langle G_{1}^{(+1 / 2)}\left(B G_{2}^{(-1 / 2)} B^{-1}\right)\right\rangle=-\left\langle G_{2}^{(+1 / 2)}\left(B G_{1}^{(-1 / 2)} B^{-1}\right)\right\rangle= \\
& \left\langle G_{4}^{(+1 / 2)}\left(B G_{3}^{(-1 / 2)} B^{-1}\right)\right\rangle=-\left\langle G_{3}^{(+1 / 2)}\left(B G_{4}^{(-1 / 2)} B^{-1}\right)\right\rangle=2 \cos \varphi \cosh \phi \cos (\theta+\chi), \\
& -\left\langle G_{1}^{(+1 / 2)}\left(B G_{3}^{(-1 / 2)} B^{-1}\right)\right\rangle=\left\langle G_{3}^{(+1 / 2)}\left(B G_{1}^{(-1 / 2)} B^{-1}\right)\right\rangle= \\
& -\left\langle G_{2}^{(+1 / 2)}\left(B G_{4}^{(-1 / 2)} B^{-1}\right)\right\rangle=\left\langle G_{4}^{(+1 / 2)}\left(B G_{2}^{(-1 / 2)} B^{-1}\right)\right\rangle=2 \sin \varphi \sinh \phi, \\
& \left\langle G_{1}^{(+1 / 2)}\left(B G_{4}^{(-1 / 2)} B^{-1}\right)\right\rangle=\left\langle G_{2}^{(+1 / 2)}\left(B G_{3}^{(-1 / 2)} B^{-1}\right)\right\rangle= \\
& \left\langle G_{4}^{(+1 / 2)}\left(B G_{1}^{(-1 / 2)} B^{-1}\right)\right\rangle=\left\langle G_{3}^{(+1 / 2)}\left(B G_{2}^{(-1 / 2)} B^{-1}\right)\right\rangle=0 \text {. }
\end{aligned}
$$




$$
\begin{gathered}
\left(B K_{1}^{(0)} B^{-1}\right)^{\|}=\sin \chi \sinh 2 \phi M_{1}^{(0)}-\cos \chi \sinh 2 \phi M_{2}^{(0)} \\
\left(B K_{2}^{(0)} B^{-1}\right)^{\|}=\cos \theta \sin 2 \varphi M_{3}^{(0)}+\sin \theta \sin 2 \varphi M_{4}^{(0)} \\
\left(B^{-1} K_{1}^{(0)} B\right)^{\|}=\sin \chi \sinh 2 \phi M_{1}^{(0)}+\cos \chi \sinh 2 \phi M_{2}^{(0)} \\
\left(B^{-1} K_{1}^{(0)} B\right)^{\|}=-\cos \theta \sin 2 \varphi M_{3}^{(0)}+\sin \theta \sin 2 \varphi M_{4}^{(0)} \\
\left(B G_{1}^{(-1 / 2)} B^{-1}\right)^{\perp}=X_{2} Y_{3} F_{1}^{(-1 / 2)}-X_{1} Y_{3} F_{2}^{(-1 / 2)}+X_{3} Y_{1} F_{3}^{(-1 / 2)}+X_{3} Y_{2} F_{4}^{(-1 / 2)} \\
\left(B G_{2}^{(-1 / 2)} B^{-1}\right)^{\perp}=X_{1} Y_{3} F_{1}^{(-1 / 2)}+X_{2} Y_{3} F_{2}^{(-1 / 2)}-X_{3} Y_{2} F_{3}^{(-1 / 2)}+X_{3} Y_{1} F_{4}^{(-1 / 2)} \\
\left(B G_{3}^{(-1 / 2)} B^{-1}\right)^{\perp}=X_{3} Y_{1} F_{1}^{(-1 / 2)}-X_{3} Y_{2} F_{2}^{(-1 / 2)}-X_{2} Y_{3} F_{3}^{(-1 / 2)}-X_{1} Y_{3} F_{4}^{(-1 / 2)} \\
\left(B G_{4}^{(-1 / 2)} B^{-1}\right)^{\perp}=X_{3} Y_{2} F_{1}^{(-1 / 2)}+X_{3} Y_{1} F_{2}^{(-1 / 2)}+X_{1} Y_{3} F_{3}^{(-1 / 2)}-X_{2} Y_{3} F_{4}^{(-1 / 2)} \\
\left(B^{-1} G_{1}^{(+1 / 2)} B\right)^{\perp}=X_{2} Y_{3} F_{1}^{(+1 / 2)}+X_{1} Y_{3} F_{2}^{(+1 / 2)}-X_{3} Y_{1} F_{3}^{(+1 / 2)}+X_{3} Y_{2} F_{4}^{(+1 / 2)} \\
\left(B^{-1} G_{2}^{(+1 / 2)} B\right)^{\perp}=-X_{1} Y_{3} F_{1}^{(+1 / 2)}+X_{2} Y_{3} F_{2}^{(+1 / 2)}-X_{3} Y_{2} F_{3}^{(+1 / 2)}-X_{3} Y_{1} F_{4}^{(+1 / 2)} \\
\left(B^{-1} G_{3}^{(+1 / 2)} B\right)^{\perp}=-X_{3} Y_{1} F_{1}^{(+1 / 2)}-X_{3} Y_{2} F_{2}^{(+1 / 2)}-X_{2} Y_{3} F_{3}^{(+1 / 2)}+X_{1} Y_{3} F_{4}^{(+1 / 2)} \\
\left(B^{-1} G_{4}^{(+1 / 2)} B\right)^{\perp}=X_{3} Y_{2} F_{1}^{(+1 / 2)}-X_{3} Y_{1} F_{2}^{(+1 / 2)}-X_{1} Y_{3} F_{3}^{(+1 / 2)}-X_{2} Y_{3} F_{4}^{(+1 / 2)}
\end{gathered}
$$

where $X_{i}, Y_{i}, i=1,2,3$ were defined in (130).

\section{References}

[1] M. Grigoriev, A. Tseytlin. Pohlmeyer reduction of $\operatorname{AdS}(5) x S(5)$ superstring sigma model. hep-th. arXiv:0711.0155. Nucl. Phys. B800 :450-501, 2008.

[2] M. Grigoriev, A. Tseytlin. On reduced Models for Superstrings on $A d S_{n} \times S^{n}$. Int. J. Mod. Phys. A23 :2107-2117, 2008. e-Print: arXiv:0806.2623 [hep-th].

[3] Olivier Babelon, Denis Bernard. Dressing Symmetries. Commun. Math. Phys. 149: 279-306, 1992. e-Print: hep-th/9111036.

[4] H. Aratyn, J.F. Gomes, A.H. Zimerman. Supersymmetry and the KDV equations for integrable hierarchies with a half integer gradation. Nucl. Phys. B676: 537-571, 2004. e-Print: hep-th/0309099.

[5] J.F. Gomes, D.M. Schmidtt, A.H. Zimerman. Super WZNW with Reductions to Supersymmetric and Fermionic Integrable Models. Nucl. Phys. B821: 553-576, 2009. arXiv: 0901.4040 [hep-th].

[6] David M. Schmidtt. Supersymmetry of Affine Toda Models as Fermionic Symmetry Flows of the Extended mKdV Hierarchy. SIGMA 6:043,2010. e-Print: arXiv:0909.3109 [hep-th].

[7] H Aratyn, L A Ferreira, J F Gomes and A H Zimerman. The complex sine-Gordon equation as a symmetry flow of the AKNS hierarchy. J. Phys. A33 :L331-339, 2000. e-Print: nlin/0007002 
[8] Timothy J. Hollowood. J. Luis Miramontes. Magnons, their Solitonic Avatars and the Pohlmeyer Reduction. JHEP 0904:060, 2009. arXiv: 0902.2405 [hep-th].

[9] Timothy J. Hollowood. J. Luis Miramontes. The AdS(5) x S(5) Semi-Symmetric Space Sine-Gordon Theory. To appear

[10] Timothy J. Hollowood. J. Luis Miramontes, David M. Schmidtt. Integrability and Supersymmetry. In progress

[11] P. Di Vecchia, V.G. Knizhnik, J.L Petersen, P. Rossi. A Supersymmetric Wess-Zumino Lagrangian In Two Dimensions. Nucl. Phys. B253 :701, 1985.

[12] J. Luis Miramontes. Pohlmeyer Reduction Revisited. JHEP 0810:087, 2008. arXiv: 0808.3365 [hep-th].

[13] Ioannis Bakas, Q-Han Park, Hyun-Jonag Shin. Lagrangian formulation of symmetric space sine-Gordon models. Phys. Lett. B372 :45-52, 1996. e-Print: hep-th/9512030.

[14] H. Aratyn, J. F. Gomes, E. Nisimov, S. Pacheva, A. H. Zimerman. Symmetry Flows, Conservation Laws and Dressing Approach to the Integrable Models. Published in *Chicago 2000, Integrable hierarchies and modern physical theories* 243-275. e-Print: nlin/0012042

[15] Nigel J. Burroughs, Mark F. de Groot, Timothy J. Hollowood, J.Luis Miramontes. Generalized Drinfeld-Sokolov hierarchies 2: The Hamiltonian structures. Commun. Math. Phys. 153:187-215,1993. hep-th/9109014

[16] F. Delduc, L. Gallot. Supersymmetric Drinfeld-Sokolov reduction. J. Math. Phys. 39, 4729 (1998). arXiv: solv-int/9802013v1.

[17] Timothy J. Hollowood, J. Luis Miramontes. Classical and Quantum Solitons in the Symmetric Space Sine-Gordon Theories. arXiv: 1012.0716 [hep-th].

[18] Andrei Mikhailov. Bihamiltonian structure of the classical superstring in $\operatorname{AdS}(5)$ x S(5). e-Print: hep-th/0609108.

[19] Andrei Mikhailov, Sakura Schafer-Nameki. Sine-Gordon-like action for the Superstring in AdS(5) x S(5). JHEP 0805:075, 2008. e-Print: arXiv: 0711.0195 [hep-th].

[20] K. Zarembo. Strings on Semisymmetric Superspaces. JHEP 1005:002, 2010. e-Print: arXiv:1003.0465 [hep-th].

[21] Mirror Symmetry. Clay Mathematics Monographs Vol 1.

[22] K. I. Kobayashi and T. Uematsu. N=2 Supersymmetric Sine-Gordon theory and Conservation Laws. Phys. Lett. B 264, 107 (1991).

[23] E. Napolitano and S. Sciuto. The N=2 Supersymmetric Generalization of the Complex Sine-Gordon Model. Phys. Lett. B113:43, 1982.

[24] Toshio Nakatsu. Supersymmetric gauged Wess-Zumino-Witten models. Prog. Theor. Phys. 87:795-798, 1992.

[25] R. Roiban, Arkady A. Tseytlin. UV finiteness of Pohlmeyer-reduced form of the AdS(5) x S(5) superstring theory. JHEP 0904:078, 2009. e-Print: arXiv:0902.2489 [hep-th]. 